\title{
Intramolecular Trapping of an Intermediate in the Reduction of Imines by a Hydroxycyclopentadienyl Ruthenium Hydride: \\ Support for a Concerted Outer Sphere Mechanism
}

\author{
Charles P. Casey*, Timothy B. Clark, and Ilia A. Guzei \\ Department of Chemistry, University of Wisconsin-Madison, Madison, Wisconsin 53706 \\ casey@,chem.wisc.edu
}

Supporting Information

\section{Contents:}

General Experimental Information................................

Experimental Procedures..........................................S-3

Analysis of Reactions Studied by ${ }^{15}$ N NMR ............................

Discussion of a Bäckvall Intermolecular Trapping Experiment.............S-16

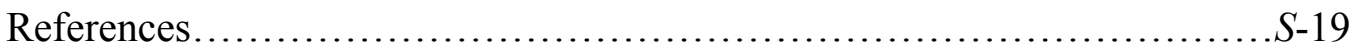

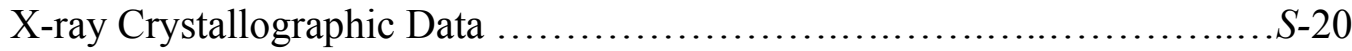

Selected ${ }^{15} \mathrm{~N}$ NMR Spectra............................................ 
General Experimental Information. All air-sensitive materials were manipulated under dry nitrogen in an Innovative Technologies glovebox or by standard high vacuum and Schlenk line techniques. Deuterated solvents and benzaldehyde were dried and distilled under nitrogen prior to use. Toluene and methylene chloride were deoxygenated and dried in a solvent purification system by passing through an activated alumina column and an oxygen scavenging column under argon. trans-1,4-Cyclohexanediamine and cis-1,4-cyclohexanediamine were purchased from TCI America and used as obtained. Benzylamine, ${ }^{15} \mathrm{~N}$-benzylamine, $\mathrm{N}$ isopropylbenzylamine, and 1,4-cyclohexanedione mono-ethylene ketal were purchased from Aldrich and used as obtained. $\left\{\left[2,5-\mathrm{Ph}_{2}-3,4-\mathrm{Tol}_{2}\left(\eta^{5}-\mathrm{C}_{4} \mathrm{CO}\right)\right]_{2} \mathrm{H}\right\} \mathrm{Ru}_{2}(\mathrm{CO})_{4}(\mu-\mathrm{H}),\left[2,5-\mathrm{Ph}_{2}-3,4-\right.$ $\left.\left.\mathrm{Tol}_{2}\left(\eta^{4}-\mathrm{C}_{4} \mathrm{COH}\right)\right]\right\} \mathrm{Ru}(\mathrm{CO})_{2} \mathrm{H} \quad(\mathbf{1}), \quad$ and $\left.\quad\left[2,5-\mathrm{Ph}_{2}-3,4-\mathrm{Tol}_{2}\left(\eta^{4}-\mathrm{C}_{4} \mathrm{CO}\right)\right] \mathrm{Ru}(\mathrm{CO})_{2}\right]_{2} \quad(\mathbf{1 4}) \quad$ were synthesized according to literature procedures. ${ }^{1}$

${ }^{1} \mathrm{H},{ }^{13} \mathrm{C}\left\{{ }^{1} \mathrm{H}\right\}$, and ${ }^{15} \mathrm{~N}\left\{{ }^{1} \mathrm{H}\right\}$ NMR spectra were obtained on a Varian Unity 500, Inova 500, or a Mercury 300 spectrometer. ${ }^{15} \mathrm{~N}\left\{{ }^{1} \mathrm{H}\right\}$ NMR spectra were obtained using an inverse gated pulse sequence with a $35 \mathrm{sec}$ relaxation delay to allow quantitative comparisons of ${ }^{15} \mathrm{~N}$ peaks by integration, and are reported on the $\delta$ scale with nitrobenzene referencing. The $2 \mathrm{D}{ }^{1} \mathrm{H}-{ }^{15} \mathrm{~N}$ HSQC spectra were typically obtained using a ${ }^{1} \mathrm{H}$ NMR spectral width of $5000 \mathrm{~Hz}$ with 2048 points, a ${ }^{15} \mathrm{~N}$ spectral width of $4000 \mathrm{~Hz}$ with 250 increments, and 16 transients with a $1.8 \mathrm{sec}$ relaxation delay was used. The spectra were processed by applying a standard Gaussian apodization followed by fourier transformation into $2048 \times 1024$ points. IR spectra were obtained on a Mattson Polaris FT-IR spectrometer. Electrospray ionization mass spectra were obtained with a Waters (Micromass) LCT (Beverly, MA) equipped with a time-of-flight analyzer. Unless otherwise indicated, samples were dissolved in methanol and sprayed with a sample cone voltage of $20 \mathrm{~V}$. For High Resolution Mass Spectra (HRMS), an aliquot of a 
known compound (lock mass) was added to the sample. Melting points were obtained using a Thomas Hoover Capillary Melting Point Apparatus and are reported uncorrected. 


\section{Experimental Procedures}

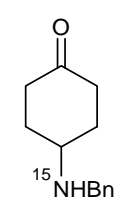

1,4-(O= $\left.\left(c-\mathbf{C}_{6} \mathbf{H}_{10}\right)^{15} \mathbf{N H B n}\right)(\mathbf{1 3}) .^{2} \quad$ A solution of 1,4 -cyclohexanedione mono-ethylene ketal $(1.00 \mathrm{~g}, 6.41 \mathrm{mmol})$ and ${ }^{15} \mathrm{~N}$-benzylamine $(0.700 \mathrm{~mL}, 6.41 \mathrm{mmol})$ in $15 \mathrm{~mL}$ of benzene containing $4 \AA$ mol sieves (ca. $1 \mathrm{~g}$ ) was heated at $50{ }^{\circ} \mathrm{C}$ for $70 \mathrm{~h}$. The reaction mixture was filtered through celite and concentrated in vacuo, followed by addition of $15 \mathrm{~mL}$ of methanol. The reaction mixture was cooled to $0{ }^{\circ} \mathrm{C}$, and $\mathrm{NaBH}_{4}(0.73 \mathrm{~g}, 19.23 \mathrm{mmol})$ was added. After 16 $\mathrm{h}$, the reaction mixture was poured over $100 \mathrm{~mL}$ of ice and acidified to $\mathrm{pH} 2$ with $2 \mathrm{M} \mathrm{HCl}$. The reaction mixture was heated at $50{ }^{\circ} \mathrm{C}$ for $2 \mathrm{~h}$ and adjusted to $\mathrm{pH} 12$ by addition of $\mathrm{NaOH}$. The resulting aqueous solution was extracted with $\mathrm{CH}_{2} \mathrm{Cl}_{2}(3 \times 50 \mathrm{~mL})$, the organics were dried over $\mathrm{Na}_{2} \mathrm{SO}_{4}$, filtered, and concentrated in vacuo. Purification by bulb-to-bulb distillation $\left(95-100{ }^{\circ} \mathrm{C}\right.$ / $0.1 \mathrm{mmHg})$ gave 13 as a pale yellow oil $(0.82 \mathrm{~g}, 63 \%) .{ }^{1} \mathrm{H} \mathrm{NMR}\left(\mathrm{CDCl}_{3}, 300 \mathrm{MHz}\right) \delta 7.33(\mathrm{~m}$, 4H), $7.25(\mathrm{~m}, 1 \mathrm{H}), 3.85(\mathrm{~s}, 2 \mathrm{H}), 3.02(\mathrm{ddd}, J=11.5,8.1,3.5 \mathrm{~Hz}, 1 \mathrm{H}), 2.53(\mathrm{ddd}, J=14.8,9.9$, 5.7 Hz, 2H), 2.29 (ddd, $J=14.8,12.2,6.8 \mathrm{~Hz}, 2 \mathrm{H}), 2.09(\mathrm{~m}, 2 \mathrm{H}), 1.75(\mathrm{~m}, 2 \mathrm{H}), 1.43$ (br s, $1 \mathrm{H})$. ${ }^{13} \mathrm{C} \mathrm{NMR}\left(\mathrm{CDCl}_{3}, 75 \mathrm{MHz}\right) \delta 211.7,140.6,128.7,128.3,127.3,53.1(\mathrm{~d}, J=4.4 \mathrm{~Hz}), 51.8(\mathrm{~d}, J=$ $4.1 \mathrm{~Hz}), 38.6(\mathrm{~d}, J=1.7 \mathrm{~Hz}), 32.2(\mathrm{~d}, J=2.3 \mathrm{~Hz}) .{ }^{15} \mathrm{~N} \mathrm{NMR}\left(\mathrm{CDCl}_{3}, 50.7 \mathrm{MHz}\right) \delta-327.73$. IR (thin film) $3030,2937,1714 \mathrm{~cm}^{-1}$. HRMS (ESI) $\mathrm{m} / z$ calcd for $\mathrm{C}_{13} \mathrm{H}_{18}{ }^{15} \mathrm{NO}(\mathrm{M}+\mathrm{H})^{+} 205.1359$, found 205.1365 . 


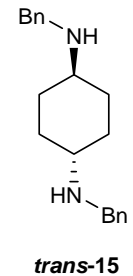

Trans-1,4-(BnNH) $)_{2}-\boldsymbol{c}-\mathbf{C}_{6} \mathbf{H}_{10}$ (trans-15). Benzaldehyde (1.12 mL, $\left.11.0 \mathrm{mmol}\right)$ was slowly added to a solution of trans-1,4-cyclohexanediamine $(0.570 \mathrm{~g}, 5.00 \mathrm{mmol})$ in $30 \mathrm{~mL}$ of methanol. After $3 \mathrm{~h}$, sodium borohydride $(0.600 \mathrm{~g}, 16.0 \mathrm{mmol})$ was added. After an additional $1.5 \mathrm{~h}$, the reaction mixture was diluted with $50 \mathrm{~mL}$ of $1.0 \mathrm{M} \mathrm{NaOH}$, and extracted with $\mathrm{Et}_{2} \mathrm{O}(2 \times$ $75 \mathrm{~mL}$ ). The organic layer was washed with $50 \mathrm{~mL}$ saturated aqueous sodium chloride, dried over $\mathrm{MgSO}_{4}$, filtered, and concentrated in vacuo. The resulting colorless solid was purified by evaporation of volatile impurities under vacuum $\left(50{ }^{\circ} \mathrm{C} / 0.1 \mathrm{~mm} \mathrm{Hg}\right)$ to give trans-15 as a colorless solid (1.22 g, 83\%), mp 64-66 ${ }^{\circ} \mathrm{C} .{ }^{1} \mathrm{H}$ NMR $\left(\mathrm{CDCl}_{3}, 500 \mathrm{MHz}\right) \delta 7.31(\mathrm{~m}, 8 \mathrm{H}), 7.24$ $(\mathrm{m}, 2 \mathrm{H}), 3.80(\mathrm{~s}, 4 \mathrm{H}), 2.51(\mathrm{~m}, 2 \mathrm{H}), 1.98(\mathrm{~d}, J=6.2 \mathrm{~Hz}, 4 \mathrm{H}), 1.45-0.94(\mathrm{br} \mathrm{s}, 2 \mathrm{H} ; \mathrm{m}, 4 \mathrm{H}) .{ }^{13} \mathrm{C}$ NMR $\left(\mathrm{CDCl}_{3}, 125 \mathrm{MHz}\right) \delta 141.0,128.6,128.3,127.1,56.5,51.6,32.2 . \quad$ IR (thin film) 3057, 3018, 2940, 2926, 2846, $1451 \mathrm{~cm}^{-1}$. HRMS (ESI) $\mathrm{m} / z$ calcd for $\mathrm{C}_{20} \mathrm{H}_{27} \mathrm{~N}_{2}(\mathrm{M}+\mathrm{H})^{+} 295.2174$, found 295.2163.

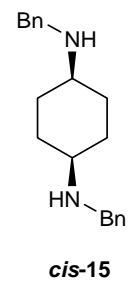

Cis-1,4-(BnNH) $)_{2}-\boldsymbol{c}-\mathbf{C}_{6} \mathbf{H}_{10}$ (cis-15). Benzaldehyde (1.12 mL, $11.0 \mathrm{mmol}$ ) was slowly added to a solution of cis-1,4-cyclohexanediamine $(0.570 \mathrm{~g}, 5.00 \mathrm{mmol})$ in $30 \mathrm{~mL}$ of methanol. 
After $3 \mathrm{~h}$, sodium borohydride $(0.600 \mathrm{~g}, 16.0 \mathrm{mmol})$ was added. After an additional $1 \mathrm{~h}$, the reaction mixture was diluted with $50 \mathrm{~mL}$ of $1.0 \mathrm{M} \mathrm{NaOH}$, and extracted with $\mathrm{Et}_{2} \mathrm{O}(2 \times 75 \mathrm{~mL})$. The organic layer was washed with $50 \mathrm{~mL}$ saturated aqueous sodium chloride, dried over $\mathrm{MgSO}_{4}$, filtered, and concentrated in vacuo. The resulting colorless oil was purified by evaporation of volatile impurities under vacuum $\left(60{ }^{\circ} \mathrm{C} / 0.1 \mathrm{~mm} \mathrm{Hg}\right)$ to give cis-15 as a colorless oil (1.30 g, 88\%). ${ }^{1} \mathrm{H}$ NMR $\left(\mathrm{CDCl}_{3}, 500 \mathrm{MHz}\right) \delta 7.33(\mathrm{~m}, 8 \mathrm{H}), 7.23(\mathrm{~m}, 2 \mathrm{H}), 3.78$ (s, 4H), $2.69(\mathrm{~m}, 2 \mathrm{H}), 1.61(\mathrm{~m}, 8 \mathrm{H}), 1.26(\mathrm{br} \mathrm{s}, 2 \mathrm{H}) .{ }^{13} \mathrm{C} \mathrm{NMR}\left(\mathrm{CDCl}_{3}, 125 \mathrm{MHz}\right) \delta 141.2,128.5$, 128.3, 127.0, 53.8, 51.3, 28.5. IR (thin film) 3313, 3061, 3026, 2929, 2851, $1425 \mathrm{~cm}^{-1}$. HRMS (ESI) $m / z$ calcd for $\mathrm{C}_{20} \mathrm{H}_{27} \mathrm{~N}_{2}(\mathrm{M}+\mathrm{H})^{+}$295.2174, found 295.2179.

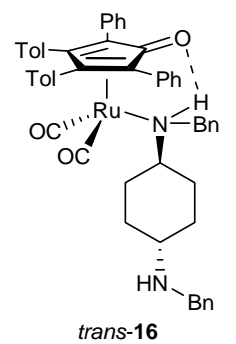

\section{$\left[2,5-\mathrm{Ph}_{2}-3,4-\mathrm{Tol}_{2}\left(\eta^{4}-\mathrm{C}_{4} \mathrm{CO}\right)\right] \mathrm{Ru}(\mathrm{CO})_{2}\left\{\right.$ trans-[1,4-(BnNH)-c- $\left.\left.\mathrm{C}_{6} \mathrm{H}_{10}(\mathrm{NHBn})\right]\right\} \quad($ trans-}

16). A slurry of $\left\{\left[2,5-\mathrm{Ph}_{2}-3,4-\mathrm{Tol}_{2}\left(\eta^{4}-\mathrm{C}_{4} \mathrm{CO}\right)\right] \mathrm{Ru}(\mathrm{CO})_{2}\right\}_{2}(0.200 \mathrm{~g}, 0.175 \mathrm{mmol})$ and trans-15 $(0.103 \mathrm{~g}, 0.350 \mathrm{mmol})$ in $5.7 \mathrm{~mL}$ of toluene was stirred for $15 \mathrm{~h}$. The reaction mixture was concentrated in vacuo and the resulting brown oil was dissolved in $1 \mathrm{~mL} \mathrm{CH}_{2} \mathrm{Cl}_{2}$, diluted with 5 $\mathrm{mL}$ pentane, and cooled to $0{ }^{\circ} \mathrm{C}$. The resulting slurry was filtered to provide bisruthenium diamine complex $23(0.058 \mathrm{~g}, 25 \%$; see below for details $)$ and a solution of trans-16. The solution was filtered through celite and evaporated under vacuum to give trans-16 (0.220 g, $73 \%$ ) as a brown solid. Crystallization of the brown solid from $\mathrm{CH}_{2} \mathrm{Cl}_{2}$ and $n$-heptane by slow partial evaporation afforded crystals suitable for X-ray crystallographic analysis, mp 122-126 
${ }^{\circ} \mathrm{C} . \quad{ }^{1} \mathrm{H}$ NMR $\left(\mathrm{C}_{7} \mathrm{D}_{8}, 500 \mathrm{MHz}\right) \delta 8.17(\mathrm{~d}, J=8.0 \mathrm{~Hz}, 2 \mathrm{H}), 8.10(\mathrm{~d}, J=9.0 \mathrm{~Hz}, 2 \mathrm{H}), 7.27(\mathrm{~m}$, 8H), $7.15(\mathrm{~m}, 8 \mathrm{H}), 7.04(\mathrm{~m}, 4 \mathrm{H}), 6.69(\mathrm{~m}, 4 \mathrm{H}), 3.85(\mathrm{dd}, J=13.2,2.2 \mathrm{~Hz}, 1 \mathrm{H}), 3.75(\mathrm{dd}, J=$ 13.5, 11.5 Hz, 1H), $3.41(\mathrm{~d}, J=13.0 \mathrm{~Hz}, 1 \mathrm{H}), 3.38(\mathrm{~d}, J=13.0 \mathrm{~Hz}, 1 \mathrm{H}), 2.41(\mathrm{~d}, J=11.2 \mathrm{~Hz}$ 1H), $2.28(\mathrm{~m}, 2 \mathrm{H}), 2.18(\mathrm{~m}, 1 \mathrm{H}), 1.93(\mathrm{~m}, 1 \mathrm{H}), 1.82(\mathrm{~s}, 3 \mathrm{H}), 1.80(\mathrm{~s}, 3 \mathrm{H}), 1.71(\mathrm{~m}, 1 \mathrm{H}), 1.37(\mathrm{~m}$, 1H), $1.00(\mathrm{~m}, 2 \mathrm{H}), 0.60(\mathrm{~m}, 2 \mathrm{H}), 0.35(\mathrm{~m}, 1 \mathrm{H}) .{ }^{13} \mathrm{C} \mathrm{NMR}\left(\mathrm{CD}_{2} \mathrm{Cl}_{2}, 125 \mathrm{MHz}\right) \delta$ 203.1, 201.1, $163.4,141.5,138.0,137.8,133.6,132.9,132.5,132.2,130.5,130.4,129.3,128.9,128.71$, $128.65,128.59,128.54,128.4,128.3,128.2,128.1,127.7,126.99,126.96,126.6,126.3,104.0$, $103.9,84.6,83.1,60.6,57.4,56.2,51.4,32.5,31.6,31.2,28.9,21.19,21.18 . \quad{ }^{1} \mathrm{H}-{ }^{15} \mathrm{~N}$ HSQC $\left(\mathrm{C}_{7} \mathrm{D}_{8}, 500 \mathrm{MHz}, 24{ }^{\circ} \mathrm{C}\right) \delta\left({ }^{15} \mathrm{~N}\right)-312.0 ;\left({ }^{1} \mathrm{H}\right)$ 2.41. IR (thin film) 3059, 3028, 2940, 2002, 1944 , 1565, 1498, $1449 \mathrm{~cm}^{-1}$. HRMS (ESI) $m / z$ calcd for $\mathrm{C}_{53} \mathrm{H}_{51} \mathrm{~N}_{2} \mathrm{O}_{3} \mathrm{Ru}(\mathrm{M}+\mathrm{H})^{+}$865.2943, found 865.2910.

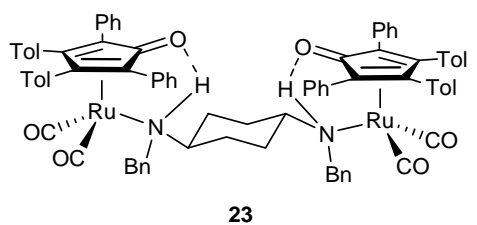

$\left\{\left[2,5-\mathrm{Ph}_{2}-3,4-\mathrm{Tol}_{2}\left(\eta^{5}-\mathrm{C}_{4} \mathrm{CO}\right)\right] \mathrm{Ru}(\mathrm{CO})_{2}\right\}_{2}\left\{\mu-\left[\operatorname{trans}-1,4-(\mathrm{BnNH})-c-\mathrm{C}_{6} \mathrm{H}_{10}(\mathrm{NHBn})\right]\right\} \quad(23)$.

Slow diffusion of $n$-pentane vapor into a $\mathrm{CH}_{2} \mathrm{Cl}_{2}$ solution of $\mathbf{2 3}$ afforded crystals suitable for $\mathrm{X}$ ray crystallographic analysis, mp $209-212{ }^{\circ} \mathrm{C} .{ }^{1} \mathrm{H}$ NMR $\left(\mathrm{C}_{6} \mathrm{D}_{6}, 300 \mathrm{MHz}\right) \delta 8.17(\mathrm{~d}, J=8.4 \mathrm{~Hz}$, 4H), $8.14(\mathrm{~d}, J=7.5 \mathrm{~Hz}, 4 \mathrm{H}), 7.32(\mathrm{~d}, J=8.1 \mathrm{~Hz}, 2 \mathrm{H}), 7.23(\mathrm{~d}, J=9.0 \mathrm{~Hz}, 10 \mathrm{H}), 7.00(\mathrm{~m}, 16 \mathrm{H})$, $6.67(\mathrm{~m}, 10 \mathrm{H}), 3.71(\mathrm{~d}, J=12.0 \mathrm{~Hz}, 2 \mathrm{H}), 3.50(\mathrm{t}, J=12.5 \mathrm{~Hz}, 2 \mathrm{H}), 2.28(\mathrm{~d}, J=10.8 \mathrm{~Hz}, 2 \mathrm{H})$, $2.11(\mathrm{~m}, 2 \mathrm{H}), 1.78(\mathrm{~s}, 6 \mathrm{H}), 1.77(\mathrm{~s}, 6 \mathrm{H}), 0.75-0.30(\mathrm{~m}, 8 \mathrm{H}) .{ }^{13} \mathrm{C}$ NMR $\left(\mathrm{C}_{6} \mathrm{D}_{6}, 75 \mathrm{MHz}\right) \delta 201.8$ (CO ${ }^{13} \mathrm{C}^{\prime}$ 's degenerate), 164.3, 138.00, 137.98, 137.7, 134.8, 133.5, 132.8, 132.6, 130.9, 130.7, $130.6,129.9,129.7,129.2,129.1,128.6,128.5,128.23,128.18,127.2,126.6,105.4,104.3,85.2$, 
82.8, 60.8, 56.6, 30.7, 28.1, 21.23, 21.20. ${ }^{1} \mathrm{H}^{15}{ }^{15} \mathrm{HSQC}\left(\mathrm{C}_{7} \mathrm{D}_{8}, 500 \mathrm{MHz}, 24{ }^{\circ} \mathrm{C}\right) \delta\left({ }^{15} \mathrm{~N}\right)$ $-310.5 ;\left({ }^{1} \mathrm{H}\right)$ 2.22. IR (thin film) 3056, 3028, $29592009,1950,1560,1490 \mathrm{~cm}^{-1}$.

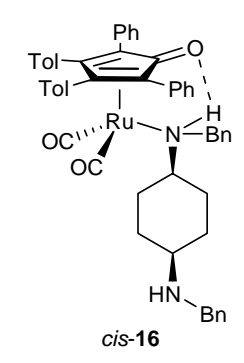

\section{$\left[2,5-\mathrm{Ph}_{2}-3,4-\mathrm{Tol}_{2}\left(\eta^{4}-\mathrm{C}_{4} \mathrm{CO}\right)\right] \mathrm{Ru}(\mathrm{CO})_{2}\left\{c i s-\left[1,4-(\mathrm{BnNH})-c-\mathrm{C}_{6} \mathrm{H}_{10}(\mathrm{NHBn})\right]\right\}$ (cis-16). A} slurry of $\left\{\left[2,5-\mathrm{Ph}_{2}-3,4-\mathrm{Tol}_{2}\left(\eta^{4}-\mathrm{C}_{4} \mathrm{CO}\right)\right] \mathrm{Ru}(\mathrm{CO})_{2}\right\}_{2}(0.200 \mathrm{~g}, 0.175 \mathrm{mmol})$ and cis-15 $(0.103 \mathrm{~g}$, $0.350 \mathrm{mmol}$ ) in $5.7 \mathrm{~mL}$ of toluene was stirred for $15 \mathrm{~h}$. The reaction mixture was concentrated in vacuo and the resulting brown oil was dissolved in $1 \mathrm{~mL} \mathrm{CH}_{2} \mathrm{Cl}_{2}$, diluted with $5 \mathrm{~mL}$ pentane, and cooled to $0{ }^{\circ} \mathrm{C}$. The resulting slurry was filtered through celite to provide a solution of cis-16. The solution was evaporated under high vacuum to give cis-16 $(0.230 \mathrm{~g}, 76 \%)$ as an orange solid, mp 202-204 ${ }^{\circ} \mathrm{C} .{ }^{1} \mathrm{H}$ NMR $\left(\mathrm{C}_{7} \mathrm{D}_{8}, 500 \mathrm{MHz}\right) \delta 8.17(\mathrm{~d}, J=7.2 \mathrm{~Hz}, 2 \mathrm{H}), 8.09(\mathrm{~d}, J=7.2$ $\mathrm{Hz}, 2 \mathrm{H}), 7.28(\mathrm{~m}, 10 \mathrm{H}), 7.21(\mathrm{~m}, 8 \mathrm{H}), 6.68(\mathrm{~m}, 6 \mathrm{H}), 3.85(\mathrm{~m}, 2 \mathrm{H}), 3.52(\mathrm{~m}, 1 \mathrm{H}), 3.42(\mathrm{~m}, 1 \mathrm{H})$, $2.46(\mathrm{~m}, 2 \mathrm{H}), 2.18(\mathrm{br} \mathrm{s}, 2 \mathrm{H}), 1.82(\mathrm{~s}, 3 \mathrm{H}), 1.79(\mathrm{~s}, 3 \mathrm{H}), 1.62(\mathrm{~m}, 1 \mathrm{H}), 1.50(\mathrm{~m}, 2 \mathrm{H}), 1.39(\mathrm{~m}$, $1 \mathrm{H}), 1.07(\mathrm{~d}, J=13.8 \mathrm{~Hz}, 1 \mathrm{H}), 0.76($ br s, $1 \mathrm{H}), 0.58(\mathrm{~m}, 1 \mathrm{H}), 0.41(\mathrm{~m}, 1 \mathrm{H}) .{ }^{13} \mathrm{C} \mathrm{NMR}\left(\mathrm{CD}_{2} \mathrm{Cl}_{2}\right.$, $125 \mathrm{MHz}) \delta 202.4,201.1,163.5,141.6,138.0,137.9,133.8,133.0,132.5,132.2,130.5,130.4$, $129.3,129.0,128.8,128.7,128.6,128.42,128.39,128.3,128.2,128.1,127.6,126.93,126.86$, 126.5, 126.2, 103.8, 103.7, 84.5, 83.1, 60.4, 58.6, 51.3, 50.4, 29.3, 28.2, 26.9, 25.0, 21.19, 21.18. ${ }^{1} \mathrm{H}-{ }^{15} \mathrm{~N}$ HSQC $\left(\mathrm{C}_{7} \mathrm{D}_{8}, 500 \mathrm{MHz}, 24{ }^{\circ} \mathrm{C}\right) \delta\left({ }^{15} \mathrm{~N}\right)-311.7,-334.6 ;\left({ }^{1} \mathrm{H}\right) 2.46,0.76$. IR (thin film) 3059, 3028, 2940, 2002, 1944, 1565, 1498, $1449 \mathrm{~cm}^{-1}$. HRMS (ESI) $\mathrm{m} / \mathrm{z}$ calcd for $\mathrm{C}_{53} \mathrm{H}_{51} \mathrm{~N}_{2} \mathrm{O}_{3} \mathrm{Ru}(\mathrm{M}+\mathrm{H})^{+}$865.2943, found 865.2890. 


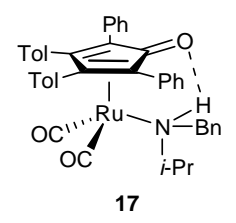

$\left[2,5-\mathrm{Ph}_{2}-3,4-\mathrm{Tol}_{2}\left(\eta^{4}-\mathrm{C}_{4} \mathrm{CO}\right)\right] \mathbf{R u}(\mathrm{CO})_{2}(\mathbf{i}-\mathrm{PrNHBn})(\mathbf{1 7}) .^{3}$ A solution of $\left[2,5-\mathrm{Ph}_{2}-\right.$ $\left.\left.3,4-\mathrm{Tol}_{2}\left(\eta^{4}-\mathrm{C}_{4} \mathrm{CO}\right)\right] \mathrm{Ru}(\mathrm{CO})_{2}\right]_{2}(0.200 \mathrm{~g}, 0.175 \mathrm{mmol})$ and $N$-isopropylbenzylamine $(0.058 \mathrm{~mL}$, $0.350 \mathrm{mmol}$ ) in $6 \mathrm{~mL}$ of $\mathrm{CH}_{2} \mathrm{Cl}_{2}$ was stirred for $3 \mathrm{~h}$ at $24{ }^{\circ} \mathrm{C}$. The reaction mixture was concentrated in vacuo and the resulting orange solid was suspended in $5 \mathrm{~mL}$ of pentane and the resulting slurry was filtered to provide $17(0.210 \mathrm{~g}, 83 \%)$ as an orange solid: $\mathrm{mp} 166-168{ }^{\circ} \mathrm{C}$. ${ }^{1} \mathrm{H}$ NMR $\left(\mathrm{CD}_{2} \mathrm{Cl}_{2}, 500 \mathrm{MHz}\right) \delta 7.71(\mathrm{~d}, J=7.1 \mathrm{~Hz}, 2 \mathrm{H}), 7.26-7.08(\mathrm{~m}, 13 \mathrm{H}), 7.04(\mathrm{~m}, 2 \mathrm{H}), 6.96$ (t, $J=7.2 \mathrm{~Hz}, 4 \mathrm{H}), 3.67$ (dd, $J=13.6,11.9 \mathrm{~Hz}, 1 \mathrm{H}), 3.53(\mathrm{dd}, J=13.7,2.6 \mathrm{~Hz}, 1 \mathrm{H}), 2.47$ (sept d, $J=6.4,1.9 \mathrm{~Hz}, 1 \mathrm{H}), 2.23(\mathrm{~s}, 6 \mathrm{H}), 1.95(\mathrm{br} \mathrm{d}, J=11.2 \mathrm{~Hz}, 1 \mathrm{H}), 1.63(\mathrm{br} \mathrm{s}, 1 \mathrm{H}), 1.08(\mathrm{~d}, J=6.3$ $\mathrm{Hz}, 3 \mathrm{H}), 0.20(\mathrm{~d}, J=6.5 \mathrm{~Hz}, 3 \mathrm{H}) .{ }^{13} \mathrm{C} \mathrm{NMR}\left(\mathrm{CD}_{2} \mathrm{Cl}_{2}, 125 \mathrm{MHz}\right) \delta 203.1,201.2,163.1,138.0$, $137.9,133.7,133.0,132.4,132.3,130.5,130.3,129.4,129.3,129.0,128.8,128.7,128.6,128.2$ 128.1, 127.6, 126.6, 126.2 ( $1 \mathrm{Ar}$ not seen due to ${ }^{13} \mathrm{C}$ overlap), 104.3, 103.7, 84.7, 83.0, 61.1, 49.3, 22.5, 21.18, 21.17, 19.6. ${ }^{1} \mathrm{H}-{ }^{15} \mathrm{~N}$ HSQC $\left(\mathrm{CD}_{2} \mathrm{Cl}_{2}, 500 \mathrm{MHz}, 24{ }^{\circ} \mathrm{C}\right) \delta\left({ }^{15} \mathrm{~N}\right)-308.0 ;\left({ }^{1} \mathrm{H}\right)$ 1.95. IR (thin film) 2947, 2002, 1945, 1560, $1498 \mathrm{~cm}^{-1}$. HRMS (ESI) $\mathrm{m} / \mathrm{z}$ calcd for $\mathrm{C}_{43} \mathrm{H}_{40} \mathrm{NO}_{3} \mathrm{Ru}(\mathrm{M}+\mathrm{H})^{+}$720.2051, found 720.2079 . 


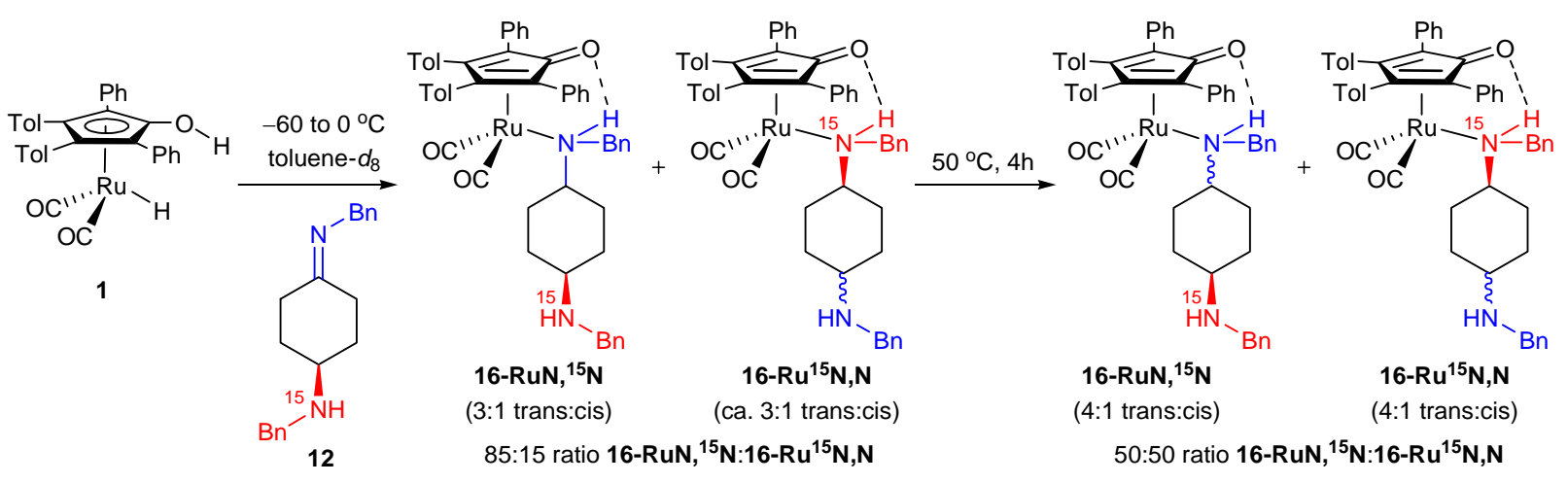

Table S-1. ${ }^{15} \mathrm{~N}$ Chemical Shifts and Isomeric Ratios from Reduction of Imine 12 in Toluene- $d_{8}{ }^{a}$

\begin{tabular}{|c|c|c|c|c|}
\hline Isomer & trans-16-RuN, ${ }^{15}$ & 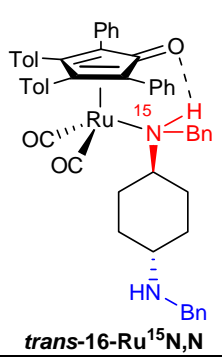 & cis-16-RuN, ${ }^{15 n}$ & 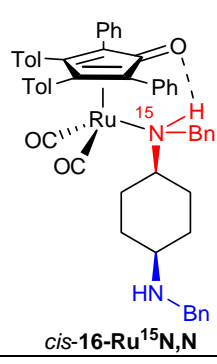 \\
\hline${ }^{15}$ N NMR Shift $\delta$ (ppm) & -324.63 & -311.29 & -334.66 & -310.95 \\
\hline$\leq 0^{\circ} \mathrm{C}$ & $64 \%$ & $12 \%$ & $21 \%$ & $\sim 3 \%^{b}$ \\
\hline After $4 \mathrm{~h}$ at $50^{\circ} \mathrm{C}^{c}$ & $40 \%$ & $40 \%$ & $10 \%$ & $10 \%$ \\
\hline
\end{tabular}

${ }^{a}$ All reactions were performed in toluene- $d_{8}$ and spectra were obtained at $0^{\circ} \mathrm{C} .{ }^{b}$ Signal/noise was not high enough to obtain accurate integrations for cis-16-Ru ${ }^{15} \mathbf{N}, \mathbf{N}$. ${ }^{c}$ Some decomposition resulted upon heating to $50{ }^{\circ} \mathrm{C}$, one side product was identified as the bisruthenium diamine complex 23, see Table S-2 and experimental procedures for details. 
${ }^{15}$ N NMR Spectral Assignments of Amine Complexes 16 (Table S-2). Two methods were used to assign the four ${ }^{15} \mathrm{~N}$ resonances of the cis and trans amine complexes $\mathbf{1 6}$ observed in the reduction of imine $\mathbf{1 2}$ by ruthenium hydride $\mathbf{1}$. The chemical shifts (at $24^{\circ} \mathrm{C}$ ) of the unbound ${ }^{15} \mathrm{~N}$-labeled amines cis-16-RuN,${ }^{15} \mathbf{N}(\delta-334.67)$ and trans-16-RuN,${ }^{\mathbf{1 5}} \mathbf{N} \quad(\delta-324.85)$ were assigned by comparison to the ${ }^{15} \mathrm{~N}$-labeled amine of imine $12(\delta-327.82)$ which has a similar chemical shift. The ${ }^{15} \mathrm{~N}$ chemical shifts of the ruthenium bound amine nitrogens of cis-16$\mathbf{R u}{ }^{15} \mathbf{N}, \mathbf{N}(\delta-311.68)$ and trans-16-Ru ${ }^{15} \mathbf{N}, \mathbf{N}(\delta-312.02)$ were assigned by comparison with amine complex $17(\delta-308.0)$, an independently synthesized amine complex that has a similar steric and electronic environment around the amine nitrogen. The difference in chemical shift between trans-16-RuN, ${ }^{15} \mathbf{N}(\delta-324.85)$ and cis-16-RuN,${ }^{15} \mathbf{N}(\delta-334.67)$ is attributed to equatorial (trans-16-RuN, $\left.{ }^{15} \mathbf{N}\right)$ and axial $\left(\right.$ cis-16-RuN,$\left.{ }^{15} \mathbf{N}\right)$ positions of the free amines. In contrast, the ruthenium bound amines of trans-16-Ru ${ }^{15} \mathbf{N}, \mathbf{N}(\delta-312.02)$ and cis-16-Ru ${ }^{15} \mathbf{N}, \mathbf{N}(\delta$ -311.68) are both in equatorial positions on the cyclohexane ring and have similar chemical shifts.

The chemical shift assignments of each isomer was also independently confirmed by the synthesis of both unlabeled trans-16 and cis-16. $2 \mathrm{D}{ }^{1} \mathrm{H}-{ }^{15} \mathrm{~N}$ HSQC spectral data was acquired on these complexes that provide cross-peaks that match the chemical shifts of the ${ }^{15} \mathrm{~N}$ NMR studied reactions. For trans-16, only one cross-peak was observed $(\delta-312.0)$ which matches that of that of trans-16-Ru ${ }^{\mathbf{1 5}} \mathbf{N}, \mathbf{N}(\delta-312.02)$ in the reduction of labeled imine 12. The coupling of the unbound nitrogen to the $\mathrm{NH}$ hydrogen averages to zero due to rapid exchange processes. This was confirmed by obtaining a non-decoupled ${ }^{15} \mathrm{~N}$ NMR spectrum of the products of reduction of 12 that showed no coupling between ${ }^{15} \mathrm{~N}$ and $\mathrm{H}$ for trans-16-RuN,${ }^{\mathbf{1 5}} \mathbf{N}$. Significant coupling of ${ }^{15} \mathrm{~N}$ and $\mathrm{H}$ was seen for the other three resonances (trans-16-Ru ${ }^{15} \mathbf{N}, \mathbf{N}$, cis-16- 
$\mathbf{R u}{ }^{15} \mathbf{N}, \mathbf{N}$, and cis-16-RuN $\left.{ }^{15} \mathbf{N}\right)$. For cis-16, two cross-peaks were observed ( $\delta-334.6$ and -311.7) which match cis-16-RuN, ${ }^{15} \mathbf{N}(\delta \quad-334.67)$ and $c i s-16-\mathbf{R u}{ }^{15} \mathbf{N}, \mathbf{N} \quad(\delta-311.68)$, respectively.

One of the decomposition products observed upon heating the products of the reduction of $\mathbf{1 2}$ was identified as bisruthenium diamine complex ${ }^{15} \mathbf{N}-\mathbf{2 3}$. Unlabeled 23 was isolated in the synthesis of unlabeled diamine complex trans-16 and characterized as described above. The 2D ${ }^{1} \mathrm{H}-{ }^{15} \mathrm{~N}$ HSQC of $23(\delta-310.5)$ was used to assign the resonance for ${ }^{15} \mathrm{~N}-23(\delta-310.61)$. 
Table S-2. Comparison with ${ }^{15} \mathrm{~N}$ Chemical Shifts of Independently Synthesized Complexes.

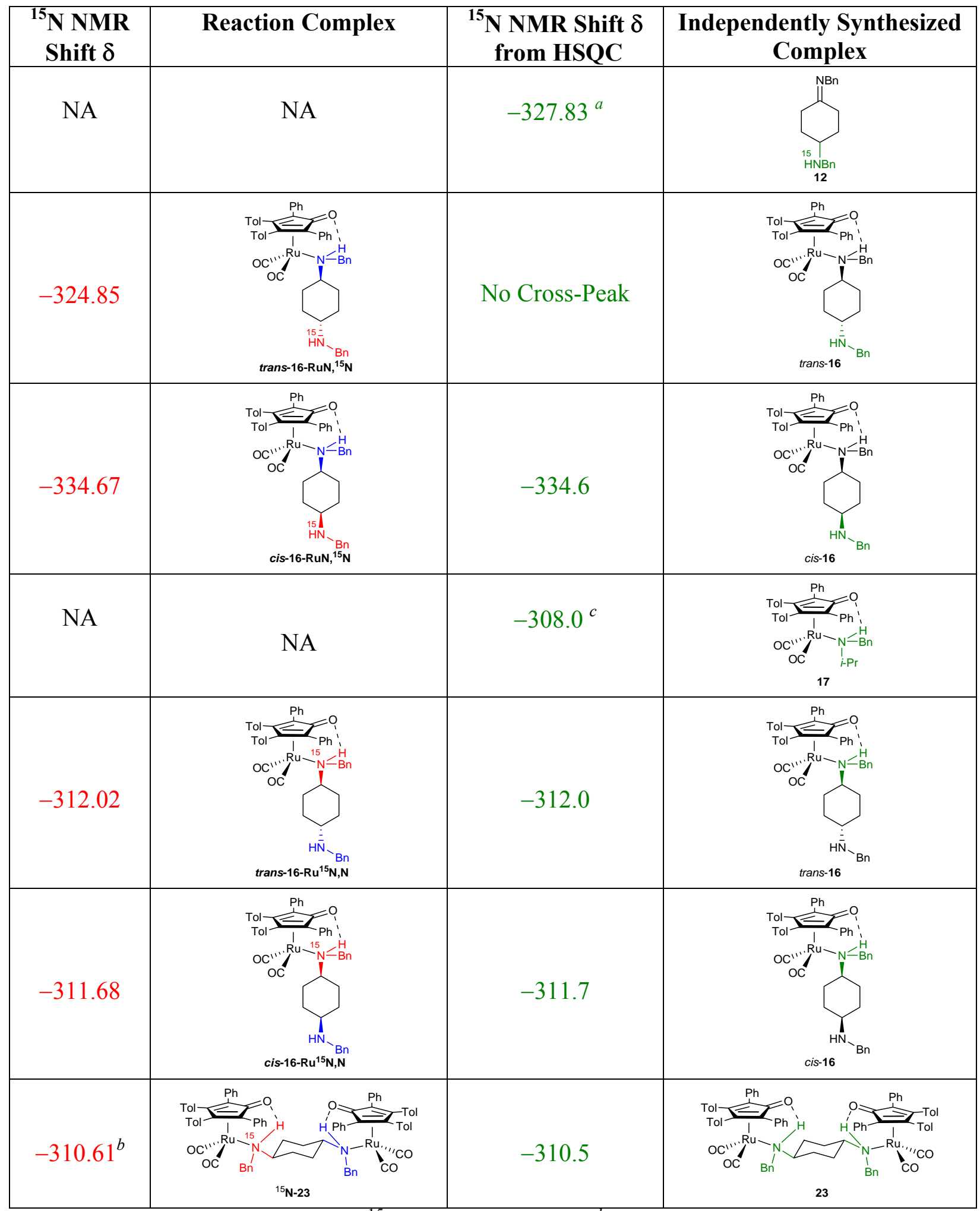

${ }^{a}$ Chemical shift determined by $1 \mathrm{D}{ }^{15} \mathrm{~N}$ NMR experiment. ${ }^{b}$ Product only appears upon warming to $50{ }^{\circ} \mathrm{C}$ for $4 \mathrm{~h}$, not present in low-temperature experiments. ${ }^{c}$ Spectra of 17 obtained in $\mathrm{CD}_{2} \mathrm{Cl}_{2}$; 17 has low solubility in toluene- $d_{8}$ and bezene- $d_{6}$. 
${ }^{15} \mathrm{~N}$ NMR Observation of Reduction of Imine 12 by 1 in $\mathrm{CD}_{2} \mathrm{Cl}_{2}$ (Table S-3). A solution of $\left[2,5-\mathrm{Ph}_{2}-3,4-\mathrm{Tol}_{2}\left(\eta^{4}-\mathrm{C}_{4} \mathrm{COH}\right)\right] \mathrm{Ru}(\mathrm{CO})_{2} \mathrm{H}(\mathbf{1})$ in $\mathrm{CD}_{2} \mathrm{Cl}_{2}$ was prepared as previously described ${ }^{1}$ and frozen in liquid nitrogen. A solution of imine $12\left(0.10 \mathrm{~mL}, 0.040 \mathrm{mmol}, 0.4 \mathrm{M}\right.$ in $\left.\mathrm{CD}_{2} \mathrm{Cl}_{2}\right)$ was added under nitrogen gas. The tube was sealed, warmed to $-78{ }^{\circ} \mathrm{C}$, and inserted into a precooled $\left(-20^{\circ} \mathrm{C}\right)$ NMR spectrometer. ${ }^{15} \mathrm{~N}$ NMR spectra at $-20{ }^{\circ} \mathrm{C}$ showed 4 resonances: $\delta-335.2$ $\left(9 \%\right.$, cis-16-RuN,$\left.{ }^{\mathbf{1 5}} \mathbf{N}\right),-322.7\left(82 \%\right.$, trans-16-RuN,$\left.{ }^{\mathbf{1 5}} \mathbf{N}\right),-311.9 \quad\left(7 \%\right.$, trans-16-Ru $\left.{ }^{15} \mathbf{N}, \mathbf{N}\right)$, $-311.3\left(\sim 2 \%\right.$, cis-16- $\left.\mathbf{R u}{ }^{15} \mathbf{N}, \mathbf{N}\right)$. The sealed NMR tube was then heated at $40{ }^{\circ} \mathrm{C}$ for $12 \mathrm{~h}$ in an oil bath. An ${ }^{15} \mathrm{~N}$ NMR spectra at $-20{ }^{\circ} \mathrm{C}$ showed a small amount of the same four resonances, a large resonance at $\delta-310.2$ due to $\left\{\left[2,5-\mathrm{Ph}_{2}-3,4-\mathrm{Tol}_{2}\left(\eta^{5}-\mathrm{C}_{4} \mathrm{CO}\right)\right] \mathrm{Ru}(\mathrm{CO})_{2}\right\}_{2}\{\mu-[$ trans-1,4$\left.\left.\left(\mathrm{Bn}^{15} \mathrm{NH}\right)-c-\mathrm{C}_{6} \mathrm{H}_{10}(\mathrm{NHBn})\right]\right\}{ }^{\mathbf{1 5}} \mathbf{N}-23$ and two substantial unassigned resonances at $\delta-322.5$ and -312.8 .
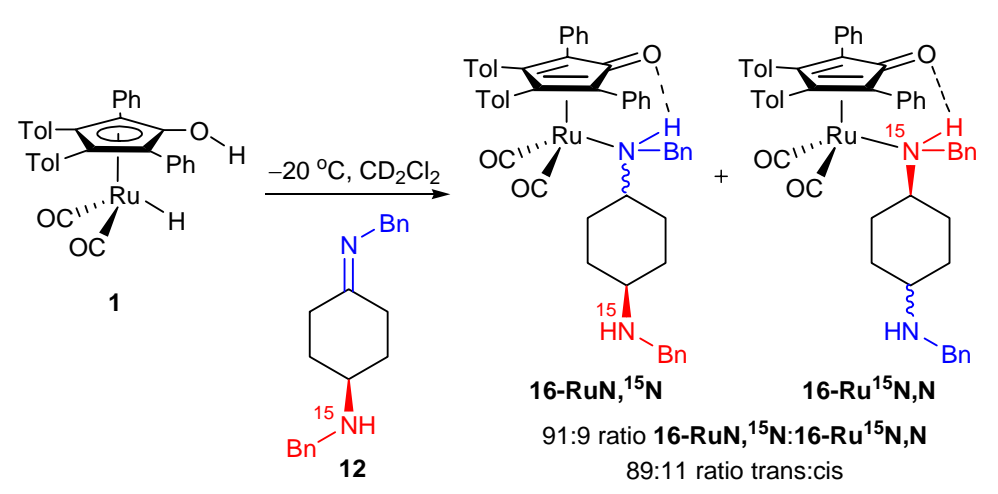

Table S-3. ${ }^{15} \mathrm{~N}$ Chemical Shifts and Isomeric Ratios from Reduction of Imine 12 in $\mathrm{CD}_{2} \mathrm{Cl}_{2}{ }^{a}$

\begin{tabular}{|c|c|c|c|}
\hline Reaction Temp $\left({ }^{\circ} \mathbf{C}\right)$ & ${ }^{15} \mathbf{N}$ NMR Shift $\delta$ & Isomer Quantity (\%) & Complex \\
\hline$\leq-20^{\circ} \mathrm{C}$ & -322.69 & 82 & trans-16-RuN, $^{\mathbf{1 5}} \mathbf{N}$ \\
\hline$\leq-20^{\circ} \mathrm{C}$ & -311.91 & 7 & trans-16-Ru $^{\mathbf{1 5}} \mathbf{N}, \mathbf{N}$ \\
\hline$\leq-20^{\circ} \mathrm{C}$ & -335.23 & 9 & cis-16-RuN, $^{\mathbf{1 5}} \mathbf{N}$ \\
\hline$\leq-20^{\circ} \mathrm{C}$ & -311.31 & $\sim 2^{b}$ & cis-16-Ru $^{\mathbf{1 5}} \mathbf{N}, \mathbf{N}$ \\
\hline
\end{tabular}

${ }^{a}$ All spectra were obtained at $-20{ }^{\circ} \mathrm{C}$ in $\mathrm{CD}_{2} \mathrm{Cl}_{2}$. Heating reactions in $\mathrm{CD}_{2} \mathrm{Cl}_{2}\left(40{ }^{\circ} \mathrm{C}\right)$ resulted in significant decomposition (primarily to ${ }^{15} \mathrm{~N}-23$ ), equilibrium ratios could not be achieved. ${ }^{b}$ Signal/noise was not high enough to obtain accurate integrations for cis-16$\mathbf{R} \mathbf{u}^{15} \mathbf{N}, \mathbf{N}$. 
Reduction of Imine 12 by 1 in the Presence of Benzylamine. Imine 12 was also reacted with 1 in the presence of benzylamine (1:1 ratio) in toluene- $d_{8}$ to determine if intermolecular kinetic trapping occurred at low temperatures (eq S-1). ${ }^{1} \mathrm{H}$ NMR spectroscopy showed less than $5 \%$ of the benzylamine complex 18 (low intensity of resonance at $\delta 3.1$ ) was formed. The major resonances seen by ${ }^{15} \mathrm{~N}$ NMR spectroscopy were $\delta-334.66$ (23\%, cis-16$\left.\mathbf{R u N},{ }^{15} \mathbf{N}\right),-324.63\left(60 \%\right.$, trans-16-RuN,$\left.{ }^{15} \mathbf{N}\right),-311.29\left(15 \%\right.$, trans-16-Ru $\left.{ }^{15} \mathbf{N}, \mathbf{N}\right)$, and -310.95 $\left(\sim 2 \%\right.$, cis-16-Ru $\left.{ }^{15} \mathbf{N}, \mathbf{N}\right)$. Upon heating at $40{ }^{\circ} \mathrm{C}$ for $1 \mathrm{~h}$, approximately $20 \%$ of $\mathbf{1 8}$ was formed concurrent with an increase from 15 to $27 \%$ of the ${ }^{15} \mathrm{~N}$ label bound to ruthenium. Thus, the rate of intramolecular scrambling of ${ }^{15} \mathrm{~N}$-label is similar to the rate of intermolecular amine exchange. Previously, we had seen more rapid intramolecular amine exchange between 7 and $\mathbf{8}$ than intermolecular amine reaction with $p$-isopropylaniline. ${ }^{4}$

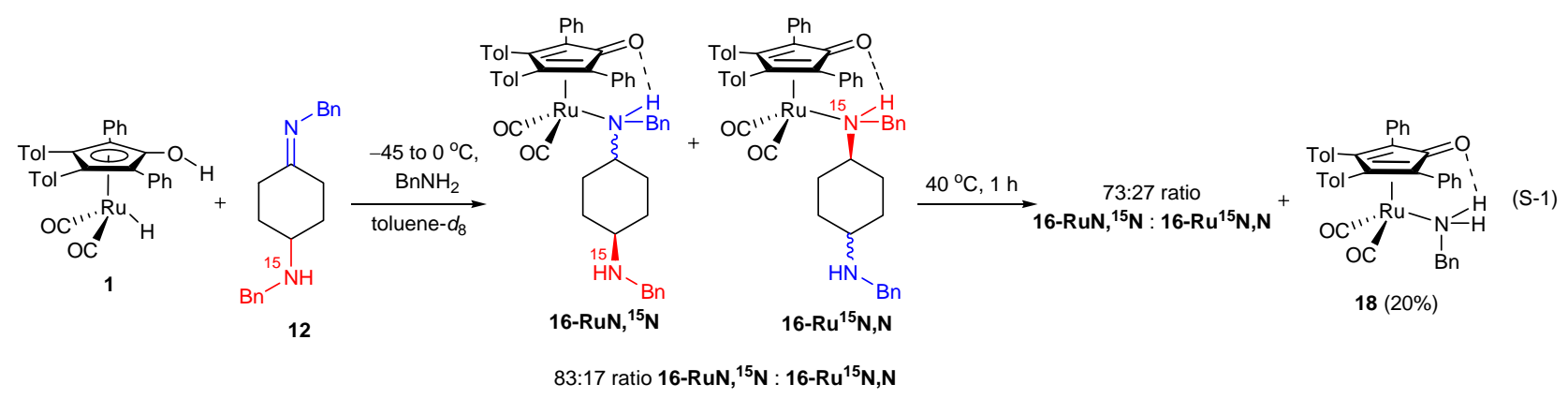


Isomerization of cis-16 to trans-16. A solution of ${ }^{15} \mathrm{~N}$ natural abundance cis-16 $(0.020$ g, $0.023 \mathrm{mmol}$ ) in $0.35 \mathrm{~mL}$ toluene- $d_{8}$ was heated at $50{ }^{\circ} \mathrm{C}$ for $2 \mathrm{~h} . \quad \mathrm{A}{ }^{1} \mathrm{H}$ NMR spectrum was obtained showing $\sim$ 4:1 ratio of trans-16:cis-16 (eq S-2).
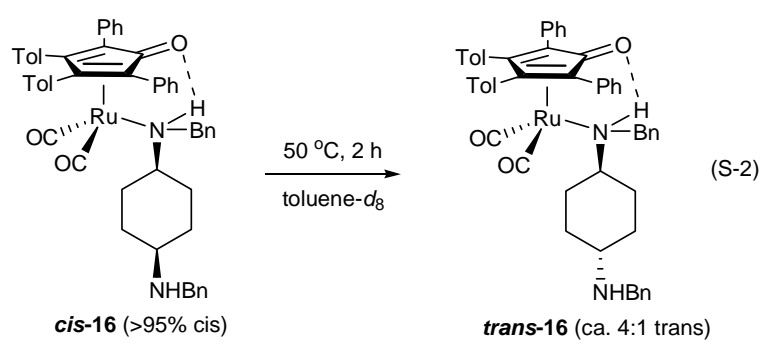

\section{Discussion of a Bäckvall Intermolecular Trapping Experiment}

In addition to intramolecular trapping experiments, Bäckvall also carried out intermolecular trapping experiments in an attempt to differentiate between the inner and outer sphere mechanisms. When imine S-25 (eq S-3) was reduced by $\mathbf{1}$ ' in the presence of amine S-26 as a trapping agent under an argon atmosphere, a 90:10 mixture of amine complexes S-27 and S28 was formed. The ratio was unchanged with varying equivalents of the trapping amine. Trapping product S-28 was not observed, however, when an atmosphere of hydrogen was used in the reaction.
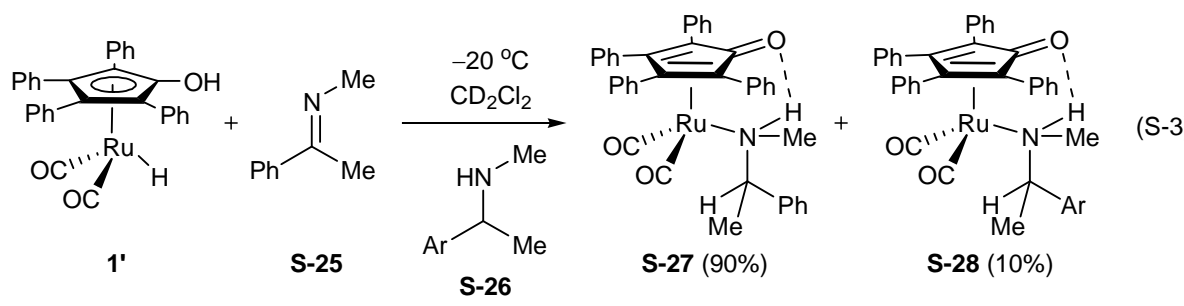

All explanations that are proposed to account for this trapping product involve a coordinatively unsaturated ruthenium intermediate (such as $\mathbf{A}$ in Scheme 1 or $\mathbf{C}$ in Scheme 2). Bäckvall considered $\mathrm{H}_{2}$ loss as one source of a coordinatively unsaturated ruthenium intermediate, ${ }^{10}$ but 
ruled out this explanation based on known slow rates of hydrogen elimination, which requires elevated temperature. ${ }^{13}$

Bäckvall suggested that an unsaturated intermediate could be formed by amine dissociation from the $\eta^{2}-16$ e- ruthenium amine complex (see Scheme 11 for a similar mechanism). We believe that this process would not be kinetically competent because it requires extremely rapid cleavage of a $\mathrm{Ru}-\mathrm{N}$ bond at low temperature and generation of a very high energy 14 eruthenium complex. It is highly unlikely that dissociation of the $\mathrm{Ru}-\mathrm{N}$ bond of such an intermediate would be much faster than the dissociation of amine from $\mathbf{1 6}-\mathbf{R u N},{ }^{\mathbf{1 5}} \mathbf{N}$ which requires heating at $50^{\circ} \mathrm{C}$.

Bäckvall considered trapping of an unsaturated intermediate formed by outer sphere reduction to be unlikely since the fraction of trapping products was unaffected by the equivalents of trapping amine.

Alternative explanations for the intermeolcular trapping are based on artifacts of the experiment. Bäckvall considered that the source of ruthenium hydride 1' was contaminated with $\left[\left(\eta^{4}-\mathrm{Ph}_{4} \mathrm{C}_{4} \mathrm{CO}\right) \mathrm{Ru}(\mathrm{CO})_{2}\right]_{2} \mathbf{1 4}^{\prime}$ (starting material used in the synthesis of $\mathbf{1}$ '), which could react with S-26 directly. Bäckvall placed an upper limit on the amount of $14^{\prime}$ as $\leq 5 \%$ based on spectroscopic data, ruling out $\mathbf{1 4}^{\prime}$ as a source of trapping products.

We believe that a different experimental artifact could explain these unusual results. The imine S-25 used in the trapping experiment might have been contaminated with $10 \%$ acetophenone (the starting material for imine synthesis and a possible imine hydrolysis product). Reduction of the acetophenone would form $10 \%$ of a labile alcohol complex, ${ }^{5,6}$ which would be rapidly converted to $10 \%$ of amine complex S-26 by alcohol dissociation followed by amine coordination. This explanation is also consistent with the failure to observe trapping product S- 
26 when reduction in carried out under an atmosphere of hydrogen. Hydrogen might effectively compete with the amine for reaction with the unsaturated intermediate formed by alcohol dissociation; this would regenerate ruthenium hydride 1'. Thus, amine trapping would be avoided because of more rapid trapping of unsaturated intermediates by hydrogen than by amines. Hydrogen has been shown to react with related coordinatively unsaturated ruthenium intermediates more rapidly than Lewis basic trapping agents. ${ }^{7}$ 


\section{References}

(1) Casey, C. P.; Singer, S. W.; Powell, D. R.; Hayashi, R. K.; Kavana, M. J. Am. Chem. Soc. 2001, 123, 1090-1100.

(2) Ketone $\mathbf{1 3}$ was previously synthesized without the ${ }^{15} \mathrm{~N}$ enrichment. Samec, J. S. M.; Éll, A. H.; Åberg, J. B.; Privalov, T.; Eriksson, L.; Bäckvall, J.-E. J. Am. Chem. Soc. 2006, $128,14293-14305$.

(3) Complex 17 was synthesized by a previously described procedure. ${ }^{1}$

(4) Casey, C. P.; Bikzhanova, G. A.; Cui, Q.; Guzei, I. A. J. Am. Chem. Soc. 2005, $127,14062-14071$.

(5) Such an alcohol complex might be formed via either an inner or outer sphere mechanism.

(6) Casey, C. P.; Bikzhanova, G. A.; Bäckvall, J.-E.; Johansson, L.; Park, J.; Kim, Y. H. Organometallics 2002, 21, 1955-1959.

(7) Casey, C. P.; Strotman, N. A.; Beetner, S. E.; Johnson, J. B.; Priebe, D. C.; Guzei, I. A. Organometallics 2006, 25, 1236-1244. 
X-ray Crystal Structure of (trans-16) $)_{2} \bullet$ heptane. Data Collection. A red crystal with approximate dimensions $0.38 \times 0.18 \times 0.15 \mathrm{~mm}$ was selected under oil under ambient conditions and attached to the tip of a nylon loop. The crystal was mounted in a stream of cold nitrogen at 100(2) $\mathrm{K}$ and centered in the X-ray beam by using a video camera. The crystal evaluation and data collection were performed on a Bruker CCD-1000 diffractometer with Mo $\mathrm{K}_{\alpha}(\lambda=0.71073$ Å) radiation and the diffractometer to crystal distance of $4.9 \mathrm{~cm}$. The initial cell constants were obtained from three series of $\omega$ scans at different starting angles. Each series consisted of 20 frames collected at intervals of $0.3^{\circ}$ in a $6^{\circ}$ range about $\omega$ with the exposure time of 10 seconds per frame. A total of 85 reflections were obtained. The reflections were successfully indexed by an automated indexing routine built in the SMART program. The final cell constants were calculated from a set of 9207 strong reflections from the actual data collection. The data were collected by using the hemisphere data collection routine. The reciprocal space was surveyed to the extent of a full sphere to a resolution of $0.80 \AA$. A total of 60002 data were harvested by collecting three sets of frames with $0.36^{\circ}$ scans in $\omega$ with an exposure time 55 sec per frame and one set of frames with $0.45^{\circ}$ scans in $\varphi$ with an exposure time $55 \mathrm{sec}$ per frame. These highly redundant datasets were corrected for Lorentz and polarization effects. The absorption correction was based on fitting a function to the empirical transmission surface as sampled by multiple equivalent measurements. [1]

Structure Solution and Refinement. The systematic absences in the diffraction data were uniquely consistent for the space group $P 2_{1} / c$ that yielded chemically reasonable and computationally stable results of refinement [1]. A successful solution by the direct methods provided most non-hydrogen atoms from the $E$-map. The remaining non-hydrogen atoms were located in an alternating series of least-squares cycles and difference Fourier maps. All non- 
hydrogen atoms were refined with anisotropic displacement coefficients. All hydrogen atoms except those attached to the nitrogen atoms were included in the structure factor calculation at idealized positions and were allowed to ride on the neighboring atoms with relative isotropic displacement coefficients. The hydrogen atoms attached to the nitrogen atoms were allowed to refine with restraints. There is one-half a heptane solvent molecule per ruthenium complex. The heptane solvent molecule resides on an inversion center and is disordered in a 50:50 ratio about it. The final least-squares refinement of 581 parameters against 11461 data with restraints resulted in residuals $R$ (based on $F^{2}$ for $I \geq 2 \sigma$ ) and $w R$ (based on $F^{2}$ for all data) of 0.0486 and 0.1414 , respectively. The final difference Fourier map was featureless. The ORTEP diagram is drawn with $50 \%$ probability ellipsoids.

\section{References}

[1] Bruker-AXS. (2000-2003) SADABS V.2.05, SAINT V.6.22, SHELXTL V.6.10

\& SMART 5.622 Software Reference Manuals. Bruker-AXS, Madison, Wisconsin, USA. 


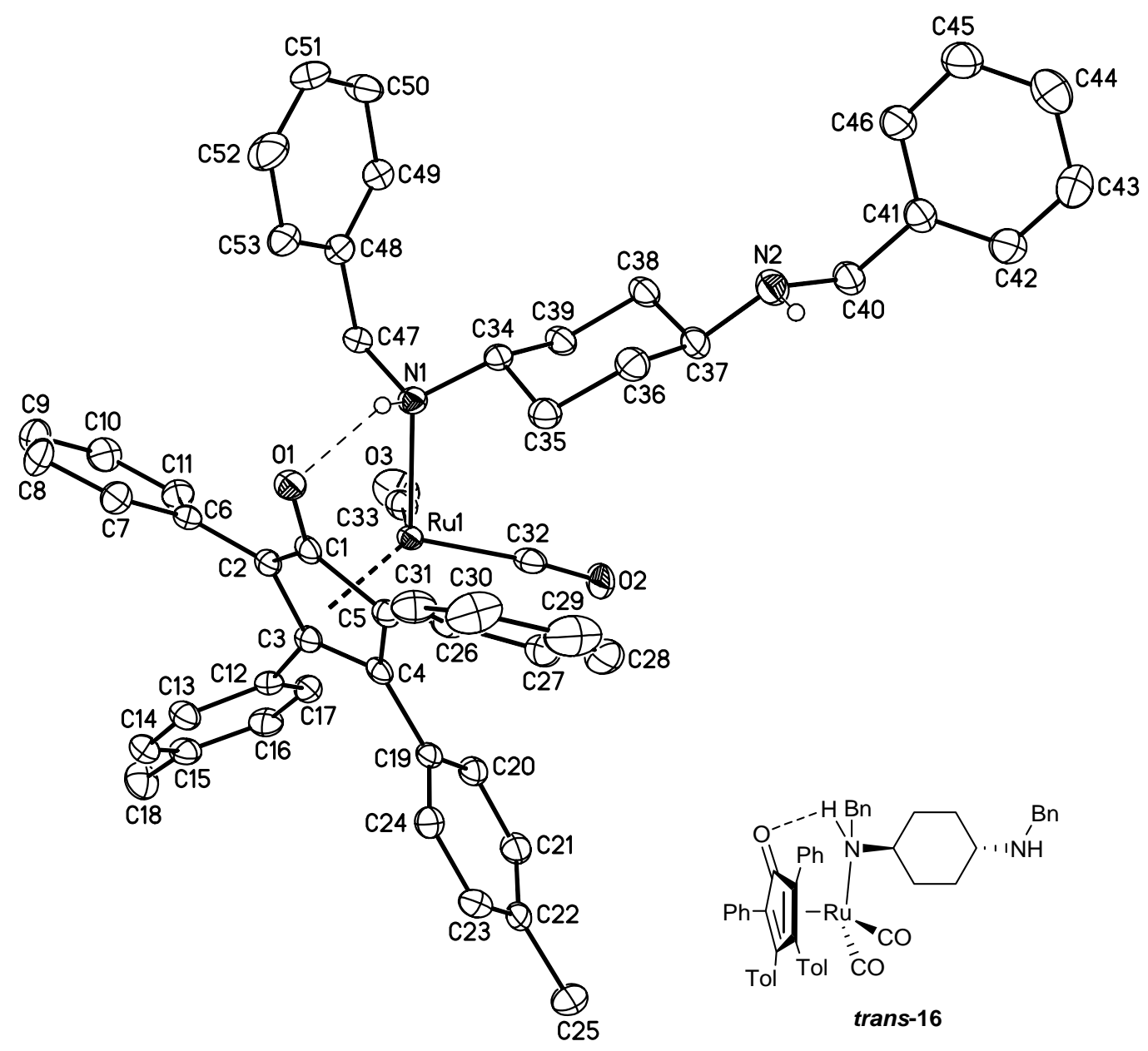

Figure S-2. ORTEP drawing of $\left[2,5-\mathrm{Ph}_{2}-3,4-\mathrm{Tol}_{2}\left(\eta^{4}-\mathrm{C}_{4} \mathrm{CO}\right)\right] \mathrm{Ru}(\mathrm{CO})_{2}\{$ trans-[1,4-(BnNH)-c$\left.\left.\mathrm{C}_{6} \mathrm{H}_{10}(\mathrm{NHBn})\right]\right\}$ [(trans-16) $)_{2} \bullet$ heptane] drawn with $50 \%$ probability ellipsoids. All hydrogen atoms except those attached to the nitrogen atoms and the solvent heptane were omitted for clarity. One intramolecular hydrogen bond is shown with a thin dashed line. 


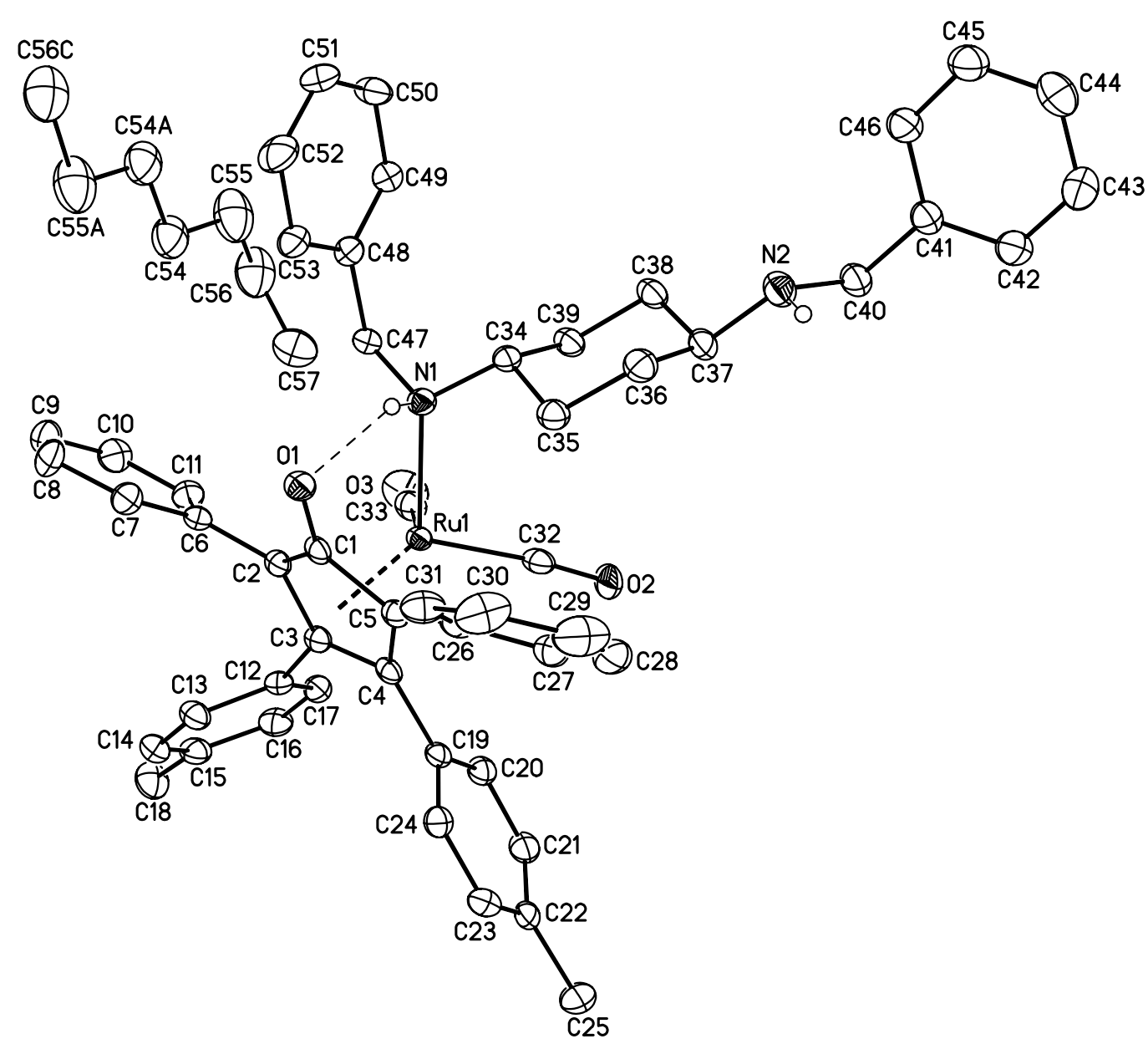

Figure S-3. ORTEP drawing of (trans-16) $)_{2} \bullet$ heptane. The ruthenium complex and the heptane solvent drawn with $50 \%$ probability ellipsoids. All hydrogen atoms except those attached to the nitrogen atoms were omitted for clarity. One intramolecular hydrogen bond is shown with a thin dashed line. The heptane solvent molecule resides on an inversion center and is equally disordered about it. Although the entire heptane solvent molecule is displayed, trans-16 crystallizes in the ratio of 2 ruthenium complexes to 1 solvent heptane molecule. Only one orientation of the heptane solvent is displayed. 
Table 1. Crystal data and structure refinement for (trans-16) $\bullet$

Empirical formula

Formula weight

Temperature

Wavelength

Crystal system

Space group

Unit cell dimensions

Volume

Z

Density (calculated)

Absorption coefficient

$\mathrm{F}(000)$

Crystal size

Theta range for data collection

Index ranges

Reflections collected

Independent reflections

Completeness to theta $=28.34^{\circ}$

Absorption correction

Max. and min. transmission

Refinement method

Data / restraints / parameters

Goodness-of-fit on $\mathrm{F}^{2}$

Final $R$ indices $[\mathrm{I}>2 \operatorname{sigma}(\mathrm{I})]$

$\mathrm{R}$ indices (all data)

Largest diff. peak and hole
$\mathrm{C}_{53} \mathrm{H}_{50} \mathrm{~N}_{2} \mathrm{O}_{3} \mathrm{Ru} \cdot 0.5 \mathrm{C}_{7} \mathrm{H}_{16}$

914.12

100(2) K

$0.71073 \AA$

Monoclinic

$\mathrm{P} 2{ }_{1} / \mathrm{c}$

$\mathrm{a}=12.0791(10) \AA \quad \alpha=90^{\circ}$.

$\mathrm{b}=13.6712(12) \AA \quad \beta=98.959(2)^{\circ}$.

$\mathrm{c}=28.205(2) \AA \quad \gamma=90^{\circ}$.

4600.9(7) $\AA^{3}$

4

$1.320 \mathrm{Mg} / \mathrm{m}^{3}$

$0.388 \mathrm{~mm}^{-1}$

1916

$0.38 \times 0.18 \times 0.15 \mathrm{~mm}^{3}$

1.46 to $28.34^{\circ}$.

$-16<=\mathrm{h}<=16,-18<=\mathrm{k}<=18,-37<=\mathrm{l}<=37$

60002

$11461[\mathrm{R}(\mathrm{int})=0.0727]$

$99.7 \%$

Multi-scan with SADABS

0.9441 and 0.8666

Full-matrix least-squares on $\mathrm{F}^{2}$

$11461 / 19 / 581$

1.058

$\mathrm{R} 1=0.0486, \mathrm{wR} 2=0.1112$

$\mathrm{R} 1=0.0887, \mathrm{wR} 2=0.1414$

0.791 and -0.766 e. $\AA^{-3}$ 
Table 2. Atomic coordinates $\left(\times 10^{4}\right)$ and equivalent isotropic displacement parameters $\left(\AA^{2} \times 10^{3}\right)$ for (trans-16) $)_{2} \bullet$ heptane. U(eq) is defined as one third of the trace of the orthogonalized $\mathrm{U}^{\mathrm{ij}}$ tensor.

\begin{tabular}{|c|c|c|c|c|}
\hline & $\mathrm{x}$ & $\mathrm{y}$ & $\mathrm{z}$ & $\mathrm{U}(\mathrm{eq})$ \\
\hline $\mathrm{Ru}(1)$ & $8692(1)$ & $3447(1)$ & $1946(1)$ & $16(1)$ \\
\hline $\mathrm{O}(1)$ & $9678(2)$ & $5445(2)$ & $1501(1)$ & $20(1)$ \\
\hline $\mathrm{O}(2)$ & $9843(2)$ & $1551(2)$ & $2288(1)$ & $31(1)$ \\
\hline $\mathrm{O}(3)$ & $6430(2)$ & $2437(2)$ & $1800(1)$ & $43(1)$ \\
\hline $\mathrm{N}(1)$ & $8910(2)$ & $3527(2)$ & 1179(1) & $19(1)$ \\
\hline $\mathrm{N}(2)$ & $12071(3)$ & $742(2)$ & $700(1)$ & $28(1)$ \\
\hline $\mathrm{C}(1)$ & 9391(3) & $5096(2)$ & $1875(1)$ & $18(1)$ \\
\hline$C(2)$ & $8253(3)$ & $5044(2)$ & 1987(1) & $17(1)$ \\
\hline$C(3)$ & $8287(3)$ & $4565(2)$ & $2448(1)$ & $18(1)$ \\
\hline C(4) & $9410(3)$ & $4228(2)$ & $2603(1)$ & $17(1)$ \\
\hline$C(5)$ & 10081(3) & $4488(2)$ & $2239(1)$ & $18(1)$ \\
\hline$C(6)$ & $7292(3)$ & $5591(2)$ & $1725(1)$ & $19(1)$ \\
\hline$C(7)$ & $7464(3)$ & $6524(2)$ & $1552(1)$ & $24(1)$ \\
\hline $\mathrm{C}(8)$ & $6575(3)$ & $7067(3)$ & $1316(1)$ & $31(1)$ \\
\hline $\mathrm{C}(9)$ & $5511(3)$ & $6684(3)$ & $1243(1)$ & $32(1)$ \\
\hline C(10) & $5322(3)$ & $5759(3)$ & $1407(1)$ & $28(1)$ \\
\hline $\mathrm{C}(11)$ & $6203(3)$ & $5220(3)$ & $1651(1)$ & $24(1)$ \\
\hline $\mathrm{C}(12)$ & $7360(3)$ & $4629(2)$ & $2736(1)$ & $19(1)$ \\
\hline C(13) & $7031(3)$ & $5565(2)$ & 2861(1) & $23(1)$ \\
\hline$C(14)$ & $6114(3)$ & $5690(3)$ & $3097(1)$ & $27(1)$ \\
\hline$C(15)$ & $5502(3)$ & 4891(3) & $3217(1)$ & $26(1)$ \\
\hline$C(16)$ & $5852(3)$ & $3963(3)$ & $3103(1)$ & $26(1)$ \\
\hline$C(17)$ & $6767(3)$ & $3831(3)$ & $2866(1)$ & $22(1)$ \\
\hline C(18) & $4468(3)$ & $5036(3)$ & $3447(1)$ & $35(1)$ \\
\hline C(19) & $9861(3)$ & $3832(2)$ & $3088(1)$ & $19(1)$ \\
\hline $\mathrm{C}(20)$ & $9415(3)$ & $3010(2)$ & $3279(1)$ & $22(1)$ \\
\hline $\mathrm{C}(21)$ & $9847(3)$ & $2675(3)$ & $3732(1)$ & $26(1)$ \\
\hline $\mathrm{C}(22)$ & $10742(3)$ & $3144(3)$ & $4013(1)$ & $26(1)$ \\
\hline$C(23)$ & 11191(3) & $3964(3)$ & $3820(1)$ & $26(1)$ \\
\hline$C(24)$ & $10752(3)$ & $4313(2)$ & $3364(1)$ & $23(1)$ \\
\hline$C(25)$ & $11238(4)$ & $2746(3)$ & 4499(1) & $37(1)$ \\
\hline$C(26)$ & $11304(3)$ & $4370(3)$ & $2259(1)$ & $22(1)$ \\
\hline $\mathrm{C}(27)$ & $11882(3)$ & $3526(3)$ & $2440(1)$ & $27(1)$ \\
\hline $\mathrm{C}(28)$ & $13021(3)$ & $3437(3)$ & $2427(1)$ & $36(1)$ \\
\hline C(29) & $13600(3)$ & $4181(4)$ & $2238(1)$ & $45(1)$ \\
\hline C(30) & $13043(3)$ & $5030(4)$ & $2080(1)$ & $40(1)$ \\
\hline C(31) & 11911(3) & $5120(3)$ & $2086(1)$ & $27(1)$ \\
\hline$C(32)$ & $9395(3)$ & $2245(2)$ & $2137(1)$ & $23(1)$ \\
\hline C(33) & $7285(3)$ & $2813(2)$ & $1840(1)$ & $26(1)$ \\
\hline C(34) & $9684(3)$ & $2848(2)$ & $974(1)$ & $19(1)$ \\
\hline$C(35)$ & $10862(3)$ & $2924(2)$ & $1256(1)$ & $23(1)$ \\
\hline C(36) & $11667(3)$ & 2297(3) & $1024(1)$ & $25(1)$ \\
\hline C(37) & $11290(3)$ & $1235(3)$ & $969(1)$ & $24(1)$ \\
\hline $\mathrm{C}(38)$ & $10098(3)$ & $1170(2)$ & $703(1)$ & $24(1)$ \\
\hline C(39) & $9285(3)$ & 1791(2) & $941(1)$ & $22(1)$ \\
\hline C(40) & $12036(3)$ & $-328(3)$ & $700(1)$ & $29(1)$ \\
\hline C(41) & $12872(3)$ & $-670(3)$ & 391(1) & $26(1)$ \\
\hline$C(42)$ & $13846(3)$ & $-1156(3)$ & $576(1)$ & $32(1)$ \\
\hline C(43) & $14643(3)$ & $-1395(3)$ & $290(1)$ & $37(1)$ \\
\hline
\end{tabular}


S-26

\begin{tabular}{lrrrr}
$\mathrm{C}(44)$ & $14462(3)$ & $-1154(3)$ & $-192(2)$ & $37(1)$ \\
$\mathrm{C}(45)$ & $13480(3)$ & $-691(3)$ & $-385(1)$ & $34(1)$ \\
$\mathrm{C}(46)$ & $12701(3)$ & $-440(3)$ & $-95(1)$ & $30(1)$ \\
$\mathrm{C}(47)$ & $7819(3)$ & $3658(2)$ & $850(1)$ & $22(1)$ \\
$\mathrm{C}(48)$ & $7952(3)$ & $3948(3)$ & $344(1)$ & $23(1)$ \\
$\mathrm{C}(49)$ & $7664(3)$ & $3300(3)$ & $-36(1)$ & $25(1)$ \\
$\mathrm{C}(50)$ & $7831(3)$ & $3560(3)$ & $-499(1)$ & $30(1)$ \\
$\mathrm{C}(51)$ & $8281(3)$ & $4455(3)$ & $-581(1)$ & $35(1)$ \\
$\mathrm{C}(52)$ & $8562(3)$ & $5108(3)$ & $-205(1)$ & $35(1)$ \\
$\mathrm{C}(53)$ & $8396(3)$ & $4856(3)$ & $258(1)$ & $29(1)$ \\
$\mathrm{C}(54)$ & $4811(4)$ & $4757(3)$ & $214(2)$ & $44(1)$ \\
$\mathrm{C}(55)$ & $4414(4)$ & $3741(4)$ & $128(2)$ & $58(1)$ \\
$\mathrm{C}(56)$ & $3841(10)$ & $3193(12)$ & $480(4)$ & $54(3)$ \\
$\mathrm{C}(57)$ & $4676(8)$ & $3059(7)$ & $936(3)$ & $44(2)$ \\
$\mathrm{C}(56 \mathrm{~A})$ & $4175(11)$ & $3341(12)$ & $620(4)$ & $54(3)$ \\
& & & & \\
\hline
\end{tabular}


Table 3. Bond lengths $[\AA]$ and angles $\left[{ }^{\circ}\right]$ for (trans-16) ${ }_{2} \bullet$ heptane.

\begin{tabular}{|c|c|c|c|}
\hline $\mathrm{Ru}(1)-\mathrm{C}(32)$ & $1.889(3)$ & $\bar{C}(20)-C(21)$ & $1.381(4)$ \\
\hline $\mathrm{Ru}(1)-\mathrm{C}(33)$ & $1.890(4)$ & $\mathrm{C}(20)-\mathrm{H}(20)$ & 0.9500 \\
\hline $\mathrm{Ru}(1)-\mathrm{C}(3)$ & $2.191(3)$ & $C(21)-C(22)$ & $1.393(5)$ \\
\hline $\mathrm{Ru}(1)-\mathrm{C}(4)$ & $2.198(3)$ & $\mathrm{C}(21)-\mathrm{H}(21)$ & 0.9500 \\
\hline $\mathrm{Ru}(1)-\mathrm{N}(1)$ & $2.223(3)$ & $\mathrm{C}(22)-\mathrm{C}(23)$ & $1.393(5)$ \\
\hline $\mathrm{Ru}(1)-\mathrm{C}(2)$ & $2.253(3)$ & $\mathrm{C}(22)-\mathrm{C}(25)$ & $1.507(5)$ \\
\hline $\mathrm{Ru}(1)-\mathrm{C}(5)$ & $2.257(3)$ & $C(23)-C(24)$ & $1.396(5)$ \\
\hline $\mathrm{Ru}(1)-\mathrm{C}(1)$ & $2.426(3)$ & $\mathrm{C}(23)-\mathrm{H}(23)$ & 0.9500 \\
\hline $\mathrm{Ru}(1)-\operatorname{cent}(\mathrm{C} 1-\mathrm{C} 5)$ & $1.900(3)$ & $\mathrm{C}(24)-\mathrm{H}(24)$ & 0.9500 \\
\hline $\mathrm{O}(1)-\mathrm{C}(1)$ & $1.255(4)$ & $\mathrm{C}(25)-\mathrm{H}(25 \mathrm{~A})$ & 0.9800 \\
\hline $\mathrm{O}(2)-\mathrm{C}(32)$ & $1.141(4)$ & $\mathrm{C}(25)-\mathrm{H}(25 \mathrm{~B})$ & 0.9800 \\
\hline $\mathrm{O}(3)-\mathrm{C}(33)$ & $1.143(4)$ & $\mathrm{C}(25)-\mathrm{H}(25 \mathrm{C})$ & 0.9800 \\
\hline $\mathrm{N}(1)-\mathrm{C}(34)$ & $1.496(4)$ & $C(26)-C(31)$ & $1.392(5)$ \\
\hline $\mathrm{N}(1)-\mathrm{C}(47)$ & $1.499(4)$ & $C(26)-C(27)$ & $1.403(5)$ \\
\hline $\mathrm{N}(1)-\mathrm{H}(1)$ & $0.88(3)$ & $\mathrm{C}(27)-\mathrm{C}(28)$ & $1.386(5)$ \\
\hline $\mathrm{N}(2)-\mathrm{C}(40)$ & $1.464(5)$ & $\mathrm{C}(27)-\mathrm{H}(27)$ & 0.9500 \\
\hline $\mathrm{N}(2)-\mathrm{C}(37)$ & $1.464(4)$ & $\mathrm{C}(28)-\mathrm{C}(29)$ & $1.388(6)$ \\
\hline $\mathrm{N}(2)-\mathrm{H}(2)$ & $0.90(3)$ & $\mathrm{C}(28)-\mathrm{H}(28)$ & 0.9500 \\
\hline$C(1)-C(2)$ & $1.459(5)$ & $C(29)-C(30)$ & $1.380(6)$ \\
\hline $\mathrm{C}(1)-\mathrm{C}(5)$ & $1.474(4)$ & $\mathrm{C}(29)-\mathrm{H}(29)$ & 0.9500 \\
\hline $\mathrm{C}(2)-\mathrm{C}(3)$ & $1.451(4)$ & $\mathrm{C}(30)-\mathrm{C}(31)$ & $1.377(5)$ \\
\hline $\mathrm{C}(2)-\mathrm{C}(6)$ & $1.477(4)$ & $\mathrm{C}(30)-\mathrm{H}(30)$ & 0.9500 \\
\hline$C(3)-C(4)$ & $1.435(4)$ & $\mathrm{C}(31)-\mathrm{H}(31)$ & 0.9500 \\
\hline $\mathrm{C}(3)-\mathrm{C}(12)$ & $1.484(4)$ & $\mathrm{C}(34)-\mathrm{C}(39)$ & $1.521(4)$ \\
\hline$C(4)-C(5)$ & $1.448(4)$ & $\mathrm{C}(34)-\mathrm{C}(35)$ & $1.522(4)$ \\
\hline$C(4)-C(19)$ & $1.491(4)$ & $\mathrm{C}(34)-\mathrm{H}(34)$ & 1.0000 \\
\hline$C(5)-C(26)$ & $1.478(5)$ & $C(35)-C(36)$ & $1.519(5)$ \\
\hline$C(6)-C(7)$ & $1.393(5)$ & $\mathrm{C}(35)-\mathrm{H}(35 \mathrm{~A})$ & 0.9900 \\
\hline$C(6)-C(11)$ & $1.395(5)$ & $\mathrm{C}(35)-\mathrm{H}(35 \mathrm{~B})$ & 0.9900 \\
\hline$C(7)-C(8)$ & $1.387(5)$ & $C(36)-C(37)$ & $1.522(5)$ \\
\hline $\mathrm{C}(7)-\mathrm{H}(7)$ & 0.9500 & $\mathrm{C}(36)-\mathrm{H}(36 \mathrm{~A})$ & 0.9900 \\
\hline $\mathrm{C}(8)-\mathrm{C}(9)$ & $1.373(5)$ & $\mathrm{C}(36)-\mathrm{H}(36 \mathrm{~B})$ & 0.9900 \\
\hline $\mathrm{C}(8)-\mathrm{H}(8)$ & 0.9500 & $\mathrm{C}(37)-\mathrm{C}(38)$ & $1.519(5)$ \\
\hline$C(9)-C(10)$ & $1.378(5)$ & $\mathrm{C}(37)-\mathrm{H}(37)$ & 1.0000 \\
\hline $\mathrm{C}(9)-\mathrm{H}(9)$ & 0.9500 & $\mathrm{C}(38)-\mathrm{C}(39)$ & $1.530(5)$ \\
\hline$C(10)-C(11)$ & $1.386(5)$ & $\mathrm{C}(38)-\mathrm{H}(38 \mathrm{~A})$ & 0.9900 \\
\hline $\mathrm{C}(10)-\mathrm{H}(10)$ & 0.9500 & $\mathrm{C}(38)-\mathrm{H}(38 \mathrm{~B})$ & 0.9900 \\
\hline $\mathrm{C}(11)-\mathrm{H}(11)$ & 0.9500 & $\mathrm{C}(39)-\mathrm{H}(39 \mathrm{~A})$ & 0.9900 \\
\hline $\mathrm{C}(12)-\mathrm{C}(17)$ & $1.386(5)$ & $\mathrm{C}(39)-\mathrm{H}(39 \mathrm{~B})$ & 0.9900 \\
\hline$C(12)-C(13)$ & $1.402(5)$ & $\mathrm{C}(40)-\mathrm{C}(41)$ & $1.507(5)$ \\
\hline$C(13)-C(14)$ & $1.388(5)$ & $\mathrm{C}(40)-\mathrm{H}(40 \mathrm{~A})$ & 0.9900 \\
\hline $\mathrm{C}(13)-\mathrm{H}(13)$ & 0.9500 & $\mathrm{C}(40)-\mathrm{H}(40 \mathrm{~B})$ & 0.9900 \\
\hline$C(14)-C(15)$ & $1.390(5)$ & $\mathrm{C}(41)-\mathrm{C}(42)$ & $1.381(5)$ \\
\hline $\mathrm{C}(14)-\mathrm{H}(14)$ & 0.9500 & $\mathrm{C}(41)-\mathrm{C}(46)$ & $1.391(5)$ \\
\hline$C(15)-C(16)$ & $1.391(5)$ & $\mathrm{C}(42)-\mathrm{C}(43)$ & $1.389(5)$ \\
\hline$C(15)-C(18)$ & $1.507(5)$ & $\mathrm{C}(42)-\mathrm{H}(42)$ & 0.9500 \\
\hline$C(16)-C(17)$ & $1.390(5)$ & $C(43)-C(44)$ & $1.383(6)$ \\
\hline $\mathrm{C}(16)-\mathrm{H}(16)$ & 0.9500 & $\mathrm{C}(43)-\mathrm{H}(43)$ & 0.9500 \\
\hline $\mathrm{C}(17)-\mathrm{H}(17)$ & 0.9500 & $\mathrm{C}(44)-\mathrm{C}(45)$ & $1.380(6)$ \\
\hline $\mathrm{C}(18)-\mathrm{H}(18 \mathrm{~A})$ & 0.9800 & $\mathrm{C}(44)-\mathrm{H}(44)$ & 0.9500 \\
\hline $\mathrm{C}(18)-\mathrm{H}(18 \mathrm{~B})$ & 0.9800 & $C(45)-C(46)$ & $1.381(5)$ \\
\hline C(18)-H(18C) & 0.9800 & $\mathrm{C}(45)-\mathrm{H}(45)$ & 0.9500 \\
\hline$C(19)-C(20)$ & $1.391(5)$ & $\mathrm{C}(46)-\mathrm{H}(46)$ & 0.9500 \\
\hline$C(19)-C(24)$ & $1.393(5)$ & $\mathrm{C}(47)-\mathrm{C}(48)$ & $1.512(4)$ \\
\hline
\end{tabular}




\begin{tabular}{|c|c|c|c|}
\hline $\mathrm{C}(47)-\mathrm{H}(47 \mathrm{~A})$ & 0.9900 & $\mathrm{C}(54)-\mathrm{H}(54 \mathrm{~A})$ & 0.9900 \\
\hline $\mathrm{C}(47)-\mathrm{H}(47 \mathrm{~B})$ & 0.9900 & $\mathrm{C}(54)-\mathrm{H}(54 \mathrm{~B})$ & 0.9900 \\
\hline $\mathrm{C}(48)-\mathrm{C}(53)$ & $1.389(5)$ & $C(55)-C(56)$ & $1.497(11)$ \\
\hline $\mathrm{C}(48)-\mathrm{C}(49)$ & $1.392(5)$ & $C(55)-C(56 A)$ & $1.560(11)$ \\
\hline$C(49)-C(50)$ & $1.400(5)$ & $\mathrm{C}(55)-\mathrm{H}(55 \mathrm{~A})$ & 0.9900 \\
\hline $\mathrm{C}(49)-\mathrm{H}(49)$ & 0.9500 & $\mathrm{C}(55)-\mathrm{H}(55 \mathrm{~B})$ & 0.9900 \\
\hline $\mathrm{C}(50)-\mathrm{C}(51)$ & $1.372(5)$ & $C(56)-C(57)$ & $1.517(11)$ \\
\hline $\mathrm{C}(50)-\mathrm{H}(50)$ & 0.9500 & $\mathrm{C}(56)-\mathrm{H}(56 \mathrm{~A})$ & 0.9900 \\
\hline $\mathrm{C}(51)-\mathrm{C}(52)$ & $1.387(5)$ & $\mathrm{C}(56)-\mathrm{H}(56 \mathrm{~B})$ & 0.9900 \\
\hline $\mathrm{C}(51)-\mathrm{H}(51)$ & 0.9500 & $\mathrm{C}(57)-\mathrm{H}(57 \mathrm{~A})$ & 0.9800 \\
\hline$C(52)-C(53)$ & $1.395(5)$ & $\mathrm{C}(57)-\mathrm{H}(57 \mathrm{~B})$ & 0.9800 \\
\hline $\mathrm{C}(52)-\mathrm{H}(52)$ & 0.9500 & $\mathrm{C}(57)-\mathrm{H}(57 \mathrm{C})$ & 0.9800 \\
\hline $\mathrm{C}(53)-\mathrm{H}(53)$ & 0.9500 & $\mathrm{C}(56 \mathrm{~A})-\mathrm{H}(56 \mathrm{C})$ & 0.9800 \\
\hline$C(54)-C(55)$ & $1.478(6)$ & $\mathrm{C}(56 \mathrm{~A})-\mathrm{H}(56 \mathrm{D})$ & 0.9800 \\
\hline $\mathrm{C}(54)-\mathrm{C}(54) \# 1$ & $1.509(8)$ & $\mathrm{C}(56 \mathrm{~A})-\mathrm{H}(56 \mathrm{E})$ & 0.9800 \\
\hline $\mathrm{C}(32)-\mathrm{Ru}(1)-\mathrm{C}(33)$ & $89.94(15)$ & $\mathrm{O}(1)-\mathrm{C}(1)-\mathrm{Ru}(1)$ & $124.6(2)$ \\
\hline $\mathrm{C}(32)-\mathrm{Ru}(1)-\mathrm{C}(3)$ & $123.90(12)$ & $\mathrm{C}(2)-\mathrm{C}(1)-\mathrm{Ru}(1)$ & $65.49(16)$ \\
\hline $\mathrm{C}(33)-\mathrm{Ru}(1)-\mathrm{C}(3)$ & $97.84(13)$ & $\mathrm{C}(5)-\mathrm{C}(1)-\mathrm{Ru}(1)$ & $65.52(16)$ \\
\hline$C(32)-R u(1)-C(4)$ & $94.60(12)$ & $\mathrm{C}(3)-\mathrm{C}(2)-\mathrm{C}(1)$ & $108.6(3)$ \\
\hline$C(33)-R u(1)-C(4)$ & $125.66(13)$ & $\mathrm{C}(3)-\mathrm{C}(2)-\mathrm{C}(6)$ & $125.9(3)$ \\
\hline $\mathrm{C}(3)-\mathrm{Ru}(1)-\mathrm{C}(4)$ & $38.18(11)$ & $\mathrm{C}(1)-\mathrm{C}(2)-\mathrm{C}(6)$ & $124.1(3)$ \\
\hline $\mathrm{C}(32)-\mathrm{Ru}(1)-\mathrm{N}(1)$ & $101.80(12)$ & $\mathrm{C}(3)-\mathrm{C}(2)-\mathrm{Ru}(1)$ & $68.62(17)$ \\
\hline $\mathrm{C}(33)-\mathrm{Ru}(1)-\mathrm{N}(1)$ & $96.27(12)$ & $\mathrm{C}(1)-\mathrm{C}(2)-\mathrm{Ru}(1)$ & $78.42(18)$ \\
\hline $\mathrm{C}(3)-\mathrm{Ru}(1)-\mathrm{N}(1)$ & $131.88(11)$ & $\mathrm{C}(6)-\mathrm{C}(2)-\mathrm{Ru}(1)$ & $129.6(2)$ \\
\hline $\mathrm{C}(4)-\mathrm{Ru}(1)-\mathrm{N}(1)$ & $134.95(11)$ & $\mathrm{C}(4)-\mathrm{C}(3)-\mathrm{C}(2)$ & $108.2(3)$ \\
\hline$C(32)-R u(1)-C(2)$ & 157.91(12) & $\mathrm{C}(4)-\mathrm{C}(3)-\mathrm{C}(12)$ & $127.6(3)$ \\
\hline $\mathrm{C}(33)-\mathrm{Ru}(1)-\mathrm{C}(2)$ & $103.86(14)$ & $\mathrm{C}(2)-\mathrm{C}(3)-\mathrm{C}(12)$ & $123.0(3)$ \\
\hline$C(3)-R u(1)-C(2)$ & $38.08(10)$ & $\mathrm{C}(4)-\mathrm{C}(3)-\mathrm{Ru}(1)$ & $71.17(17)$ \\
\hline $\mathrm{C}(4)-\mathrm{Ru}(1)-\mathrm{C}(2)$ & $63.36(11)$ & $\mathrm{C}(2)-\mathrm{C}(3)-\mathrm{Ru}(1)$ & $73.30(17)$ \\
\hline $\mathrm{N}(1)-\mathrm{Ru}(1)-\mathrm{C}(2)$ & $93.84(10)$ & $\mathrm{C}(12)-\mathrm{C}(3)-\mathrm{Ru}(1)$ & $131.1(2)$ \\
\hline$C(32)-R u(1)-C(5)$ & $99.68(13)$ & $C(3)-C(4)-C(5)$ & $108.1(3)$ \\
\hline$C(33)-R u(1)-C(5)$ & $161.12(13)$ & $C(3)-C(4)-C(19)$ & $126.1(3)$ \\
\hline $\mathrm{C}(3)-\mathrm{Ru}(1)-\mathrm{C}(5)$ & $63.31(12)$ & $\mathrm{C}(5)-\mathrm{C}(4)-\mathrm{C}(19)$ & $125.1(3)$ \\
\hline$C(4)-R u(1)-C(5)$ & $37.92(11)$ & $\mathrm{C}(3)-\mathrm{C}(4)-\mathrm{Ru}(1)$ & $70.65(17)$ \\
\hline $\mathrm{N}(1)-\mathrm{Ru}(1)-\mathrm{C}(5)$ & $97.57(11)$ & $\mathrm{C}(5)-\mathrm{C}(4)-\mathrm{Ru}(1)$ & $73.27(17)$ \\
\hline $\mathrm{C}(2)-\mathrm{Ru}(1)-\mathrm{C}(5)$ & $62.48(11)$ & $\mathrm{C}(19)-\mathrm{C}(4)-\mathrm{Ru}(1)$ & $129.6(2)$ \\
\hline$C(32)-R u(1)-C(1)$ & $133.21(13)$ & $C(4)-C(5)-C(1)$ & $108.3(3)$ \\
\hline $\mathrm{C}(33)-\mathrm{Ru}(1)-\mathrm{C}(1)$ & $136.84(13)$ & $\mathrm{C}(4)-\mathrm{C}(5)-\mathrm{C}(26)$ & $127.7(3)$ \\
\hline $\mathrm{C}(3)-\mathrm{Ru}(1)-\mathrm{C}(1)$ & $61.31(11)$ & $\mathrm{C}(1)-\mathrm{C}(5)-\mathrm{C}(26)$ & $122.8(3)$ \\
\hline $\mathrm{C}(4)-\mathrm{Ru}(1)-\mathrm{C}(1)$ & $61.39(10)$ & $\mathrm{C}(4)-\mathrm{C}(5)-\mathrm{Ru}(1)$ & $68.82(17)$ \\
\hline $\mathrm{N}(1)-\mathrm{Ru}(1)-\mathrm{C}(1)$ & $77.25(10)$ & $\mathrm{C}(1)-\mathrm{C}(5)-\mathrm{Ru}(1)$ & $78.03(18)$ \\
\hline $\mathrm{C}(2)-\mathrm{Ru}(1)-\mathrm{C}(1)$ & $36.09(11)$ & $C(26)-C(5)-R u(1)$ & $128.8(2)$ \\
\hline $\mathrm{C}(5)-\mathrm{Ru}(1)-\mathrm{C}(1)$ & $36.46(11)$ & $\mathrm{C}(7)-\mathrm{C}(6)-\mathrm{C}(11)$ & $117.9(3)$ \\
\hline $\mathrm{C}(34)-\mathrm{N}(1)-\mathrm{C}(47)$ & $112.1(2)$ & $C(7)-C(6)-C(2)$ & $119.4(3)$ \\
\hline $\mathrm{C}(34)-\mathrm{N}(1)-\mathrm{Ru}(1)$ & $121.43(19)$ & $\mathrm{C}(11)-\mathrm{C}(6)-\mathrm{C}(2)$ & $122.7(3)$ \\
\hline $\mathrm{C}(47)-\mathrm{N}(1)-\mathrm{Ru}(1)$ & 112.49(19) & $C(8)-C(7)-C(6)$ & $120.8(3)$ \\
\hline $\mathrm{C}(34)-\mathrm{N}(1)-\mathrm{H}(1)$ & $102(2)$ & $\mathrm{C}(8)-\mathrm{C}(7)-\mathrm{H}(7)$ & 119.6 \\
\hline $\mathrm{C}(47)-\mathrm{N}(1)-\mathrm{H}(1)$ & $105(2)$ & $\mathrm{C}(6)-\mathrm{C}(7)-\mathrm{H}(7)$ & 119.6 \\
\hline $\mathrm{Ru}(1)-\mathrm{N}(1)-\mathrm{H}(1)$ & $102(2)$ & $\mathrm{C}(9)-\mathrm{C}(8)-\mathrm{C}(7)$ & $120.3(3)$ \\
\hline $\mathrm{C}(40)-\mathrm{N}(2)-\mathrm{C}(37)$ & $116.1(3)$ & $\mathrm{C}(9)-\mathrm{C}(8)-\mathrm{H}(8)$ & 119.8 \\
\hline $\mathrm{C}(40)-\mathrm{N}(2)-\mathrm{H}(2)$ & $106(3)$ & $\mathrm{C}(7)-\mathrm{C}(8)-\mathrm{H}(8)$ & 119.8 \\
\hline $\mathrm{C}(37)-\mathrm{N}(2)-\mathrm{H}(2)$ & $110(3)$ & $\mathrm{C}(8)-\mathrm{C}(9)-\mathrm{C}(10)$ & $119.9(3)$ \\
\hline $\mathrm{O}(1)-\mathrm{C}(1)-\mathrm{C}(2)$ & $126.4(3)$ & $\mathrm{C}(8)-\mathrm{C}(9)-\mathrm{H}(9)$ & 120.0 \\
\hline $\mathrm{O}(1)-\mathrm{C}(1)-\mathrm{C}(5)$ & $126.8(3)$ & $\mathrm{C}(10)-\mathrm{C}(9)-\mathrm{H}(9)$ & 120.0 \\
\hline $\mathrm{C}(2)-\mathrm{C}(1)-\mathrm{C}(5)$ & $105.8(3)$ & $\mathrm{C}(9)-\mathrm{C}(10)-\mathrm{C}(11)$ & $120.0(3)$ \\
\hline
\end{tabular}




\begin{tabular}{|c|c|}
\hline $\mathrm{C}(9)-\mathrm{C}(10)-\mathrm{H}(10)$ & 120.0 \\
\hline $\mathrm{C}(11)-\mathrm{C}(10)-\mathrm{H}(10)$ & 120.0 \\
\hline$C(10)-C(11)-C(6)$ & $121.0(3)$ \\
\hline $\mathrm{C}(10)-\mathrm{C}(11)-\mathrm{H}(11)$ & 119.5 \\
\hline $\mathrm{C}(6)-\mathrm{C}(11)-\mathrm{H}(11)$ & 119.5 \\
\hline $\mathrm{C}(17)-\mathrm{C}(12)-\mathrm{C}(13)$ & $118.2(3)$ \\
\hline $\mathrm{C}(17)-\mathrm{C}(12)-\mathrm{C}(3)$ & $124.3(3)$ \\
\hline$C(13)-C(12)-C(3)$ & $117.4(3)$ \\
\hline $\mathrm{C}(14)-\mathrm{C}(13)-\mathrm{C}(12)$ & $120.8(3)$ \\
\hline $\mathrm{C}(14)-\mathrm{C}(13)-\mathrm{H}(13)$ & 119.6 \\
\hline $\mathrm{C}(12)-\mathrm{C}(13)-\mathrm{H}(13)$ & 119.6 \\
\hline $\mathrm{C}(13)-\mathrm{C}(14)-\mathrm{C}(15)$ & $121.0(3)$ \\
\hline $\mathrm{C}(13)-\mathrm{C}(14)-\mathrm{H}(14)$ & 119.5 \\
\hline $\mathrm{C}(15)-\mathrm{C}(14)-\mathrm{H}(14)$ & 119.5 \\
\hline $\mathrm{C}(14)-\mathrm{C}(15)-\mathrm{C}(16)$ & $117.9(3)$ \\
\hline $\mathrm{C}(14)-\mathrm{C}(15)-\mathrm{C}(18)$ & $120.7(3)$ \\
\hline $\mathrm{C}(16)-\mathrm{C}(15)-\mathrm{C}(18)$ & $121.4(3)$ \\
\hline $\mathrm{C}(17)-\mathrm{C}(16)-\mathrm{C}(15)$ & $121.6(3)$ \\
\hline $\mathrm{C}(17)-\mathrm{C}(16)-\mathrm{H}(16)$ & 119.2 \\
\hline $\mathrm{C}(15)-\mathrm{C}(16)-\mathrm{H}(16)$ & 119.2 \\
\hline $\mathrm{C}(12)-\mathrm{C}(17)-\mathrm{C}(16)$ & $120.5(3)$ \\
\hline $\mathrm{C}(12)-\mathrm{C}(17)-\mathrm{H}(17)$ & 119.7 \\
\hline $\mathrm{C}(16)-\mathrm{C}(17)-\mathrm{H}(17)$ & 119.7 \\
\hline $\mathrm{C}(15)-\mathrm{C}(18)-\mathrm{H}(18 \mathrm{~A})$ & 109.5 \\
\hline $\mathrm{C}(15)-\mathrm{C}(18)-\mathrm{H}(18 \mathrm{~B})$ & 109.5 \\
\hline $\mathrm{H}(18 \mathrm{~A})-\mathrm{C}(18)-\mathrm{H}(18 \mathrm{~B})$ & 109.5 \\
\hline $\mathrm{C}(15)-\mathrm{C}(18)-\mathrm{H}(18 \mathrm{C})$ & 109.5 \\
\hline $\mathrm{H}(18 \mathrm{~A})-\mathrm{C}(18)-\mathrm{H}(18 \mathrm{C})$ & 109.5 \\
\hline $\mathrm{H}(18 \mathrm{~B})-\mathrm{C}(18)-\mathrm{H}(18 \mathrm{C})$ & 109.5 \\
\hline $\mathrm{C}(20)-\mathrm{C}(19)-\mathrm{C}(24)$ & $118.4(3)$ \\
\hline$C(20)-C(19)-C(4)$ & $122.8(3)$ \\
\hline$C(24)-C(19)-C(4)$ & $118.8(3)$ \\
\hline $\mathrm{C}(21)-\mathrm{C}(20)-\mathrm{C}(19)$ & $120.9(3)$ \\
\hline $\mathrm{C}(21)-\mathrm{C}(20)-\mathrm{H}(20)$ & 119.5 \\
\hline $\mathrm{C}(19)-\mathrm{C}(20)-\mathrm{H}(20)$ & 119.5 \\
\hline $\mathrm{C}(20)-\mathrm{C}(21)-\mathrm{C}(22)$ & $121.5(3)$ \\
\hline $\mathrm{C}(20)-\mathrm{C}(21)-\mathrm{H}(21)$ & 119.3 \\
\hline $\mathrm{C}(22)-\mathrm{C}(21)-\mathrm{H}(21)$ & 119.3 \\
\hline $\mathrm{C}(23)-\mathrm{C}(22)-\mathrm{C}(21)$ & $117.5(3)$ \\
\hline $\mathrm{C}(23)-\mathrm{C}(22)-\mathrm{C}(25)$ & $121.5(3)$ \\
\hline $\mathrm{C}(21)-\mathrm{C}(22)-\mathrm{C}(25)$ & $120.9(3)$ \\
\hline $\mathrm{C}(22)-\mathrm{C}(23)-\mathrm{C}(24)$ & $121.4(3)$ \\
\hline $\mathrm{C}(22)-\mathrm{C}(23)-\mathrm{H}(23)$ & 119.3 \\
\hline $\mathrm{C}(24)-\mathrm{C}(23)-\mathrm{H}(23)$ & 119.3 \\
\hline $\mathrm{C}(19)-\mathrm{C}(24)-\mathrm{C}(23)$ & $120.3(3)$ \\
\hline $\mathrm{C}(19)-\mathrm{C}(24)-\mathrm{H}(24)$ & 119.9 \\
\hline $\mathrm{C}(23)-\mathrm{C}(24)-\mathrm{H}(24)$ & 119.9 \\
\hline $\mathrm{C}(22)-\mathrm{C}(25)-\mathrm{H}(25 \mathrm{~A})$ & 109.5 \\
\hline $\mathrm{C}(22)-\mathrm{C}(25)-\mathrm{H}(25 \mathrm{~B})$ & 109.5 \\
\hline $\mathrm{H}(25 \mathrm{~A})-\mathrm{C}(25)-\mathrm{H}(25 \mathrm{~B})$ & 109.5 \\
\hline $\mathrm{C}(22)-\mathrm{C}(25)-\mathrm{H}(25 \mathrm{C})$ & 109.5 \\
\hline $\mathrm{H}(25 \mathrm{~A})-\mathrm{C}(25)-\mathrm{H}(25 \mathrm{C})$ & 109.5 \\
\hline $\mathrm{H}(25 \mathrm{~B})-\mathrm{C}(25)-\mathrm{H}(25 \mathrm{C})$ & 109.5 \\
\hline $\mathrm{C}(31)-\mathrm{C}(26)-\mathrm{C}(27)$ & $118.3(3)$ \\
\hline$C(31)-C(26)-C(5)$ & $119.0(3)$ \\
\hline$C(27)-C(26)-C(5)$ & $122.8(3)$ \\
\hline
\end{tabular}

\begin{tabular}{|c|c|}
\hline$C(28)-C(27)-C(26)$ & $120.2(4)$ \\
\hline $\mathrm{C}(28)-\mathrm{C}(27)-\mathrm{H}(27)$ & 119.9 \\
\hline $\mathrm{C}(26)-\mathrm{C}(27)-\mathrm{H}(27)$ & 119.9 \\
\hline $\mathrm{C}(27)-\mathrm{C}(28)-\mathrm{C}(29)$ & $120.5(4)$ \\
\hline $\mathrm{C}(27)-\mathrm{C}(28)-\mathrm{H}(28)$ & 119.8 \\
\hline $\mathrm{C}(29)-\mathrm{C}(28)-\mathrm{H}(28)$ & 119.8 \\
\hline $\mathrm{C}(30)-\mathrm{C}(29)-\mathrm{C}(28)$ & $119.4(4)$ \\
\hline $\mathrm{C}(30)-\mathrm{C}(29)-\mathrm{H}(29)$ & 120.3 \\
\hline $\mathrm{C}(28)-\mathrm{C}(29)-\mathrm{H}(29)$ & 120.3 \\
\hline $\mathrm{C}(31)-\mathrm{C}(30)-\mathrm{C}(29)$ & $120.4(4)$ \\
\hline $\mathrm{C}(31)-\mathrm{C}(30)-\mathrm{H}(30)$ & 119.8 \\
\hline $\mathrm{C}(29)-\mathrm{C}(30)-\mathrm{H}(30)$ & 119.8 \\
\hline $\mathrm{C}(30)-\mathrm{C}(31)-\mathrm{C}(26)$ & 121.1(4) \\
\hline $\mathrm{C}(30)-\mathrm{C}(31)-\mathrm{H}(31)$ & 119.4 \\
\hline $\mathrm{C}(26)-\mathrm{C}(31)-\mathrm{H}(31)$ & 119.4 \\
\hline $\mathrm{O}(2)-\mathrm{C}(32)-\mathrm{Ru}(1)$ & $174.6(3)$ \\
\hline $\mathrm{O}(3)-\mathrm{C}(33)-\mathrm{Ru}(1)$ & $176.7(3)$ \\
\hline $\mathrm{N}(1)-\mathrm{C}(34)-\mathrm{C}(39)$ & $113.7(3)$ \\
\hline $\mathrm{N}(1)-\mathrm{C}(34)-\mathrm{C}(35)$ & $110.2(3)$ \\
\hline $\mathrm{C}(39)-\mathrm{C}(34)-\mathrm{C}(35)$ & $110.9(3)$ \\
\hline $\mathrm{N}(1)-\mathrm{C}(34)-\mathrm{H}(34)$ & 107.2 \\
\hline $\mathrm{C}(39)-\mathrm{C}(34)-\mathrm{H}(34)$ & 107.2 \\
\hline $\mathrm{C}(35)-\mathrm{C}(34)-\mathrm{H}(34)$ & 107.2 \\
\hline$C(36)-C(35)-C(34)$ & $110.2(3)$ \\
\hline $\mathrm{C}(36)-\mathrm{C}(35)-\mathrm{H}(35 \mathrm{~A})$ & 109.6 \\
\hline $\mathrm{C}(34)-\mathrm{C}(35)-\mathrm{H}(35 \mathrm{~A})$ & 109.6 \\
\hline $\mathrm{C}(36)-\mathrm{C}(35)-\mathrm{H}(35 \mathrm{~B})$ & 109.6 \\
\hline $\mathrm{C}(34)-\mathrm{C}(35)-\mathrm{H}(35 \mathrm{~B})$ & 109.6 \\
\hline $\mathrm{H}(35 \mathrm{~A})-\mathrm{C}(35)-\mathrm{H}(35 \mathrm{~B})$ & 108.1 \\
\hline $\mathrm{C}(35)-\mathrm{C}(36)-\mathrm{C}(37)$ & $112.3(3)$ \\
\hline $\mathrm{C}(35)-\mathrm{C}(36)-\mathrm{H}(36 \mathrm{~A})$ & 109.1 \\
\hline $\mathrm{C}(37)-\mathrm{C}(36)-\mathrm{H}(36 \mathrm{~A})$ & 109.1 \\
\hline $\mathrm{C}(35)-\mathrm{C}(36)-\mathrm{H}(36 \mathrm{~B})$ & 109.1 \\
\hline $\mathrm{C}(37)-\mathrm{C}(36)-\mathrm{H}(36 \mathrm{~B})$ & 109.1 \\
\hline $\mathrm{H}(36 \mathrm{~A})-\mathrm{C}(36)-\mathrm{H}(36 \mathrm{~B})$ & 107.9 \\
\hline $\mathrm{N}(2)-\mathrm{C}(37)-\mathrm{C}(38)$ & $110.7(3)$ \\
\hline $\mathrm{N}(2)-\mathrm{C}(37)-\mathrm{C}(36)$ & $106.5(3)$ \\
\hline $\mathrm{C}(38)-\mathrm{C}(37)-\mathrm{C}(36)$ & $110.5(3)$ \\
\hline $\mathrm{N}(2)-\mathrm{C}(37)-\mathrm{H}(37)$ & 109.7 \\
\hline $\mathrm{C}(38)-\mathrm{C}(37)-\mathrm{H}(37)$ & 109.7 \\
\hline $\mathrm{C}(36)-\mathrm{C}(37)-\mathrm{H}(37)$ & 109.7 \\
\hline $\mathrm{C}(37)-\mathrm{C}(38)-\mathrm{C}(39)$ & $112.0(3)$ \\
\hline $\mathrm{C}(37)-\mathrm{C}(38)-\mathrm{H}(38 \mathrm{~A})$ & 109.2 \\
\hline $\mathrm{C}(39)-\mathrm{C}(38)-\mathrm{H}(38 \mathrm{~A})$ & 109.2 \\
\hline $\mathrm{C}(37)-\mathrm{C}(38)-\mathrm{H}(38 \mathrm{~B})$ & 109.2 \\
\hline $\mathrm{C}(39)-\mathrm{C}(38)-\mathrm{H}(38 \mathrm{~B})$ & 109.2 \\
\hline $\mathrm{H}(38 \mathrm{~A})-\mathrm{C}(38)-\mathrm{H}(38 \mathrm{~B})$ & 107.9 \\
\hline $\mathrm{C}(34)-\mathrm{C}(39)-\mathrm{C}(38)$ & $109.5(3)$ \\
\hline $\mathrm{C}(34)-\mathrm{C}(39)-\mathrm{H}(39 \mathrm{~A})$ & 109.8 \\
\hline $\mathrm{C}(38)-\mathrm{C}(39)-\mathrm{H}(39 \mathrm{~A})$ & 109.8 \\
\hline $\mathrm{C}(34)-\mathrm{C}(39)-\mathrm{H}(39 \mathrm{~B})$ & 109.8 \\
\hline $\mathrm{C}(38)-\mathrm{C}(39)-\mathrm{H}(39 \mathrm{~B})$ & 109.8 \\
\hline $\mathrm{H}(39 \mathrm{~A})-\mathrm{C}(39)-\mathrm{H}(39 \mathrm{~B})$ & 108.2 \\
\hline $\mathrm{N}(2)-\mathrm{C}(40)-\mathrm{C}(41)$ & $106.8(3)$ \\
\hline $\mathrm{N}(2)-\mathrm{C}(40)-\mathrm{H}(40 \mathrm{~A})$ & 110.4 \\
\hline $\mathrm{C}(41)-\mathrm{C}(40)-\mathrm{H}(40 \mathrm{~A})$ & 110.4 \\
\hline
\end{tabular}




$\begin{array}{ll} & \\ \text { N(2)-C(40)-H(40B) } & 110.4 \\ \text { C(41)-C(40)-H(40B) } & 110.4 \\ \text { H(40A)-C(40)-H(40B) } & 108.6 \\ \text { C(42)-C(41)-C(46) } & 118.0(3) \\ \text { C(42)-C(41)-C(40) } & 122.5(3) \\ \text { C(46)-C(41)-C(40) } & 119.4(3) \\ \text { C(41)-C(42)-C(43) } & 121.4(3) \\ \text { C(41)-C(42)-H(42) } & 119.3 \\ \text { C(43)-C(42)-H(42) } & 119.3 \\ \text { C(44)-C(43)-C(42) } & 119.8(4) \\ \text { C(44)-C(43)-H(43) } & 120.1 \\ \text { C(42)-C(43)-H(43) } & 120.1 \\ \text { C(45)-C(44)-C(43) } & 119.4(4) \\ \text { C(45)-C(44)-H(44) } & 120.3 \\ \text { C(43)-C(44)-H(44) } & 120.3 \\ \text { C(44)-C(45)-C(46) } & 120.3(4) \\ \text { C(44)-C(45)-H(45) } & 119.9 \\ \text { C(46)-C(45)-H(45) } & 119.9 \\ \text { C(45)-C(46)-C(41) } & 121.1(4) \\ \text { C(45)-C(46)-H(46) } & 119.5 \\ \text { C(41)-C(46)-H(46) } & 119.5 \\ \text { N(1)-C(47)-C(48) } & 113.8(3) \\ \text { N(1)-C(47)-H(47A) } & 108.8 \\ \text { C(48)-C(47)-H(47A) } & 108.8 \\ \text { N(1)-C(47)-H(47B) } & 108.8 \\ \text { C(48)-C(47)-H(47B) } & 108.8 \\ \text { H(47A)-C(47)-H(47B) } & 107.7 \\ \text { C(53)-C(48)-C(49) } & 119.4(3) \\ \text { C(53)-C(48)-C(47) } & 120.0(3) \\ \text { C(49)-C(48)-C(47) } & 120.5(3) \\ \text { C(48)-C(49)-C(50) } & 120.1(3) \\ \text { C(48)-C(49)-H(49) } & 119.9 \\ \text { C(50)-C(49)-H(49) } & 119.9 \\ \text { C(51)-C(50)-C(49) } & 120.2(3) \\ \text { C(51)-C(50)-H(50) } & 119.9 \\ \text { C(49)-C(50)-H(50) } & 119.9 \\ \text { C(50)-C(51)-C(52) } & 120.0(3) \\ \text { C(50)-C(51)-H(51) } & 120.0 \\ \text { C(52)-C(51)-H(51) } & 120.0 \\ \text { C(51)-C(52)-C(53) } & 120.2(4) \\ \text { C(51)-C(52)-H(52) } & 119.9 \\ \text { C(53)-C(52)-H(52) } & 119.9 \\ \text { C(48)-C(53)-C(52) } & 120.0(3) \\ \text { C(48)-C(53)-H(53) } & 120.0 \\ \text { C(52)-C(53)-H(53) } & 120.0 \\ \text { C(55)-C(54)-C(54)\#1 } & 114.4(5) \\ \text { C(55)-C(54)-H(54A) } & 108.7 \\ \text { C(54)\#1-C(54)-H(54A) } & 108.7 \\ \text { C(55)-C(54)-H(54B) } & 108.7 \\ \text { C(54)\#1-C(54)-H(54B) } & 108.7 \\ \text { H(54A)-C(54)-H(54B) } & 107.6 \\ \text { C(54)-C(55)-C(56) } & 122.2(7) \\ \text { C(54)-C(55)-C(56A) } & 106.5(7) \\ \text { C(56)-C(55)-C(56A) } & 20.8(7) \\ \text { C(54)-C(55)-H(55A) } & 106.8 \\ \text { C(56)-C(55)-H(55A) } & 106.8 \\ & \end{array}$

$\mathrm{C}(56 \mathrm{~A})-\mathrm{C}(55)-\mathrm{H}(55 \mathrm{~A}) \quad 101.7$

$\mathrm{C}(54)-\mathrm{C}(55)-\mathrm{H}(55 \mathrm{~B}) \quad 106.8$

$\mathrm{C}(56)-\mathrm{C}(55)-\mathrm{H}(55 \mathrm{~B}) \quad 106.8$

$\mathrm{C}(56 \mathrm{~A})-\mathrm{C}(55)-\mathrm{H}(55 \mathrm{~B}) \quad 127.0$

$\mathrm{H}(55 \mathrm{~A})-\mathrm{C}(55)-\mathrm{H}(55 \mathrm{~B}) \quad 106.6$

$\mathrm{C}(57)-\mathrm{C}(56)-\mathrm{C}(55) \quad 108.0(8)$

$\mathrm{C}(57)-\mathrm{C}(56)-\mathrm{H}(56 \mathrm{~A}) \quad 110.1$

$\mathrm{C}(55)-\mathrm{C}(56)-\mathrm{H}(56 \mathrm{~A}) \quad 110.1$

$\mathrm{C}(57)-\mathrm{C}(56)-\mathrm{H}(56 \mathrm{~B}) \quad 110.1$

$\mathrm{C}(55)-\mathrm{C}(56)-\mathrm{H}(56 \mathrm{~B}) \quad 110.1$

$\mathrm{H}(56 \mathrm{~A})-\mathrm{C}(56)-\mathrm{H}(56 \mathrm{~B}) \quad 108.4$

$\mathrm{C}(55)-\mathrm{C}(56 \mathrm{~A})-\mathrm{H}(56 \mathrm{C}) \quad 109.5$

$\mathrm{C}(55)-\mathrm{C}(56 \mathrm{~A})-\mathrm{H}(56 \mathrm{D}) \quad 109.5$

$\mathrm{H}(56 \mathrm{C})-\mathrm{C}(56 \mathrm{~A})-\mathrm{H}(56 \mathrm{D}) \quad 109.5$

$\mathrm{C}(55)-\mathrm{C}(56 \mathrm{~A})-\mathrm{H}(56 \mathrm{E}) \quad 109.5$

$\mathrm{H}(56 \mathrm{C})-\mathrm{C}(56 \mathrm{~A})-\mathrm{H}(56 \mathrm{E}) \quad 109.5$

$\mathrm{H}(56 \mathrm{D})-\mathrm{C}(56 \mathrm{~A})-\mathrm{H}(56 \mathrm{E}) \quad 109.5$

Symmetry transformations used to generate

equivalent atoms: $\quad \# 1-\mathrm{x}+1,-\mathrm{y}+1,-\mathrm{z}$ 
Table 4. Anisotropic displacement parameters $\left(\AA^{2} \times 10^{3}\right)$ for (trans-16) ${ }_{2} \bullet$ heptane. The anisotropic displacement factor exponent takes the form: $-2 \pi^{2}\left[\mathrm{~h}^{2} \mathrm{a}^{* 2} \mathrm{U}^{11}+\ldots+2 \mathrm{hk} \mathrm{a} \mathrm{a}^{*} \mathrm{~b}^{12}\right]$

\begin{tabular}{|c|c|c|c|c|c|c|}
\hline & $\mathrm{U}^{11}$ & $\mathrm{U}^{22}$ & $\mathrm{U}^{33}$ & $\mathrm{U}^{23}$ & $\mathrm{U}^{13}$ & $\mathrm{U}^{12}$ \\
\hline $\mathrm{Ru}(1)$ & $22(1)$ & $12(1)$ & $13(1)$ & $1(1)$ & $5(1)$ & $-1(1)$ \\
\hline $\mathrm{O}(1)$ & $28(1)$ & $17(1)$ & $18(1)$ & $2(1)$ & $8(1)$ & $-2(1)$ \\
\hline $\mathrm{O}(2)$ & $48(2)$ & $16(1)$ & $29(1)$ & $4(1)$ & $8(1)$ & $8(1)$ \\
\hline $\mathrm{O}(3)$ & $38(2)$ & $42(2)$ & $50(2)$ & $-7(1)$ & 11(1) & $-20(1)$ \\
\hline $\mathrm{N}(1)$ & 24(1) & $17(1)$ & $16(1)$ & 1(1) & $4(1)$ & $-1(1)$ \\
\hline $\mathrm{N}(2)$ & $32(2)$ & $25(2)$ & $28(2)$ & 1(1) & 11(1) & $4(1)$ \\
\hline$C(1)$ & $26(2)$ & 11(1) & $18(1)$ & $-2(1)$ & $7(1)$ & $-1(1)$ \\
\hline$C(2)$ & $24(2)$ & 13(1) & $16(1)$ & $0(1)$ & $6(1)$ & $-2(1)$ \\
\hline$C(3)$ & $24(2)$ & $12(1)$ & $16(1)$ & $0(1)$ & $5(1)$ & $-3(1)$ \\
\hline$C(4)$ & $24(2)$ & 11(1) & $16(1)$ & $-2(1)$ & $7(1)$ & $-2(1)$ \\
\hline$C(5)$ & $23(2)$ & $14(2)$ & $17(1)$ & $-1(1)$ & $4(1)$ & $-2(1)$ \\
\hline C(6) & $25(2)$ & 21(2) & $14(1)$ & $-1(1)$ & $6(1)$ & $3(1)$ \\
\hline$C(7)$ & $29(2)$ & $22(2)$ & $23(2)$ & $2(1)$ & $5(1)$ & $0(2)$ \\
\hline$C(8)$ & $40(2)$ & $23(2)$ & $30(2)$ & $9(2)$ & $5(2)$ & $8(2)$ \\
\hline$C(9)$ & $33(2)$ & $34(2)$ & $27(2)$ & $3(2)$ & $4(2)$ & $14(2)$ \\
\hline$C(10)$ & 21(2) & $37(2)$ & $29(2)$ & $-1(2)$ & $8(1)$ & $5(2)$ \\
\hline$C(11)$ & $29(2)$ & $25(2)$ & $21(2)$ & $4(1)$ & $9(1)$ & $0(2)$ \\
\hline$C(12)$ & $22(2)$ & $22(2)$ & $13(1)$ & $0(1)$ & $3(1)$ & 1(1) \\
\hline$C(13)$ & $29(2)$ & $21(2)$ & $20(2)$ & $-2(1)$ & $8(1)$ & $-2(1)$ \\
\hline$C(14)$ & $33(2)$ & $27(2)$ & $21(2)$ & $-3(1)$ & $6(1)$ & $6(2)$ \\
\hline$C(15)$ & $27(2)$ & $33(2)$ & $18(2)$ & $-2(1)$ & $6(1)$ & $4(2)$ \\
\hline$C(16)$ & $29(2)$ & $29(2)$ & $20(2)$ & $2(1)$ & $7(1)$ & $-5(2)$ \\
\hline$C(17)$ & $27(2)$ & $19(2)$ & $19(2)$ & 1(1) & $4(1)$ & $0(1)$ \\
\hline$C(18)$ & $36(2)$ & $36(2)$ & $36(2)$ & $0(2)$ & $19(2)$ & $5(2)$ \\
\hline$C(19)$ & $27(2)$ & $16(2)$ & $14(1)$ & $0(1)$ & $7(1)$ & $3(1)$ \\
\hline$C(20)$ & $27(2)$ & $20(2)$ & $19(2)$ & 1(1) & $7(1)$ & $2(1)$ \\
\hline$C(21)$ & $37(2)$ & $22(2)$ & $20(2)$ & $3(1)$ & 11(1) & $4(2)$ \\
\hline$C(22)$ & $40(2)$ & $22(2)$ & $16(2)$ & 1(1) & $7(1)$ & $13(2)$ \\
\hline$C(23)$ & $33(2)$ & $24(2)$ & $20(2)$ & $-5(1)$ & 1(1) & $1(2)$ \\
\hline$C(24)$ & $31(2)$ & $16(2)$ & $22(2)$ & $0(1)$ & $2(1)$ & 1(1) \\
\hline$C(25)$ & $58(3)$ & $34(2)$ & $19(2)$ & $5(2)$ & $2(2)$ & $11(2)$ \\
\hline$C(26)$ & $23(2)$ & $26(2)$ & $16(1)$ & $-6(1)$ & $3(1)$ & $-1(1)$ \\
\hline$C(27)$ & $28(2)$ & $29(2)$ & $24(2)$ & $-6(1)$ & 1(1) & $2(2)$ \\
\hline$C(28)$ & $28(2)$ & 51(3) & $27(2)$ & $-7(2)$ & $-4(2)$ & $13(2)$ \\
\hline$C(29)$ & $22(2)$ & $82(4)$ & $32(2)$ & $-5(2)$ & $6(2)$ & $-1(2)$ \\
\hline$C(30)$ & $28(2)$ & 61(3) & $31(2)$ & $6(2)$ & $5(2)$ & $-10(2)$ \\
\hline$C(31)$ & $25(2)$ & $37(2)$ & $20(2)$ & $0(1)$ & $7(1)$ & $-7(2)$ \\
\hline$C(32)$ & $34(2)$ & $20(2)$ & $15(1)$ & $-1(1)$ & $7(1)$ & $-4(2)$ \\
\hline$C(33)$ & $37(2)$ & $19(2)$ & $24(2)$ & $-2(1)$ & $6(2)$ & $-3(2)$ \\
\hline$C(34)$ & $26(2)$ & $18(2)$ & $15(1)$ & $2(1)$ & $6(1)$ & $2(1)$ \\
\hline$C(35)$ & $26(2)$ & $22(2)$ & $20(2)$ & $0(1)$ & $5(1)$ & $-1(1)$ \\
\hline$C(36)$ & $24(2)$ & $28(2)$ & $24(2)$ & $0(1)$ & $6(1)$ & $1(2)$ \\
\hline C(37) & $31(2)$ & $23(2)$ & $22(2)$ & 1(1) & $10(1)$ & $7(2)$ \\
\hline$C(38)$ & $34(2)$ & $17(2)$ & $21(2)$ & $-2(1)$ & $9(1)$ & $0(1)$ \\
\hline C(39) & $28(2)$ & 19(2) & $21(2)$ & $-3(1)$ & $6(1)$ & $-1(1)$ \\
\hline$C(40)$ & $41(2)$ & $23(2)$ & $25(2)$ & $2(1)$ & $11(2)$ & $7(2)$ \\
\hline$C(41)$ & $30(2)$ & $23(2)$ & $25(2)$ & $-2(1)$ & $6(1)$ & $2(2)$ \\
\hline$C(42)$ & $40(2)$ & $28(2)$ & $26(2)$ & $-1(2)$ & $5(2)$ & $4(2)$ \\
\hline$C(43)$ & $35(2)$ & $37(2)$ & $38(2)$ & $2(2)$ & $1(2)$ & $9(2)$ \\
\hline
\end{tabular}




\section{S-32}

\begin{tabular}{llllccc}
$\mathrm{C}(44)$ & $34(2)$ & $40(2)$ & $41(2)$ & $-4(2)$ & $16(2)$ & $1(2)$ \\
$\mathrm{C}(45)$ & $44(2)$ & $31(2)$ & $27(2)$ & $1(2)$ & $9(2)$ & $1(2)$ \\
$\mathrm{C}(46)$ & $38(2)$ & $26(2)$ & $26(2)$ & $-1(2)$ & $6(2)$ & $2(2)$ \\
$\mathrm{C}(47)$ & $28(2)$ & $22(2)$ & $16(1)$ & $-1(1)$ & $4(1)$ & $4(1)$ \\
$\mathrm{C}(48)$ & $25(2)$ & $24(2)$ & $20(2)$ & $2(1)$ & $4(1)$ & $5(1)$ \\
$\mathrm{C}(49)$ & $30(2)$ & $24(2)$ & $22(2)$ & $1(1)$ & $4(1)$ & $3(1)$ \\
$\mathrm{C}(50)$ & $35(2)$ & $38(2)$ & $18(2)$ & $-4(2)$ & $5(1)$ & $3(2)$ \\
$\mathrm{C}(51)$ & $49(2)$ & $39(2)$ & $17(2)$ & $8(2)$ & $7(2)$ & $8(2)$ \\
$\mathrm{C}(52)$ & $46(2)$ & $30(2)$ & $29(2)$ & $11(2)$ & $6(2)$ & $-3(2)$ \\
$\mathrm{C}(53)$ & $42(2)$ & $22(2)$ & $22(2)$ & $3(1)$ & $2(2)$ & $3(2)$ \\
$\mathrm{C}(54)$ & $42(3)$ & $44(3)$ & $48(3)$ & $6(2)$ & $18(2)$ & $11(2)$ \\
$\mathrm{C}(55)$ & $53(3)$ & $48(3)$ & $80(4)$ & $5(3)$ & $31(3)$ & $8(2)$ \\
$\mathrm{C}(56)$ & $44(6)$ & $50(4)$ & $73(6)$ & $7(4)$ & $22(4)$ & $3(4)$ \\
$\mathrm{C}(57)$ & $42(5)$ & $48(5)$ & $43(5)$ & $-8(4)$ & $8(4)$ & $-11(4)$ \\
$\mathrm{C}(56 \mathrm{~A})$ & $44(6)$ & $50(4)$ & $73(6)$ & $7(4)$ & $22(4)$ & $3(4)$ \\
& & & & & & \\
\hline
\end{tabular}


Table 5. Hydrogen coordinates ( $\left.\times 10^{4}\right)$ and isotropic displacement parameters $\left(\AA^{2} \times 10^{3}\right)$ for (trans-16) $)_{2} \bullet$ heptane.

\begin{tabular}{|c|c|c|c|c|}
\hline & $\mathrm{x}$ & $\mathrm{y}$ & $\mathrm{z}$ & $\mathrm{U}(\mathrm{eq})$ \\
\hline $\mathrm{H}(1)$ & $9260(30)$ & $4090(20)$ & $1168(13)$ & 23 \\
\hline $\mathrm{H}(2)$ & $12780(30)$ & $910(30)$ & $821(14)$ & 33 \\
\hline $\mathrm{H}(7)$ & 8199 & 6792 & 1596 & 29 \\
\hline $\mathrm{H}(8)$ & 6703 & 7706 & 1204 & 37 \\
\hline $\mathrm{H}(9)$ & 4905 & 7058 & 1080 & 38 \\
\hline $\mathrm{H}(10)$ & 4588 & 5490 & 1352 & 34 \\
\hline $\mathrm{H}(11)$ & 6063 & 4589 & 1770 & 29 \\
\hline $\mathrm{H}(13)$ & 7441 & 6121 & 2784 & 28 \\
\hline $\mathrm{H}(14)$ & 5901 & 6330 & 3176 & 32 \\
\hline $\mathrm{H}(16)$ & 5455 & 3407 & 3188 & 31 \\
\hline $\mathrm{H}(17)$ & 6988 & 3188 & 2793 & 26 \\
\hline $\mathrm{H}(18 \mathrm{~A})$ & 3801 & 5000 & 3201 & 52 \\
\hline $\mathrm{H}(18 \mathrm{~B})$ & 4500 & 5678 & 3603 & 52 \\
\hline $\mathrm{H}(18 \mathrm{C})$ & 4431 & 4523 & 3687 & 52 \\
\hline $\mathrm{H}(20)$ & 8805 & 2674 & 3096 & 26 \\
\hline $\mathrm{H}(21)$ & 9528 & 2112 & 3855 & 31 \\
\hline $\mathrm{H}(23)$ & 11808 & 4293 & 4002 & 32 \\
\hline $\mathrm{H}(24)$ & 11063 & 4881 & 3242 & 28 \\
\hline $\mathrm{H}(25 \mathrm{~A})$ & 10658 & 2395 & 4638 & 56 \\
\hline $\mathrm{H}(25 \mathrm{~B})$ & 11527 & 3287 & 4710 & 56 \\
\hline $\mathrm{H}(25 \mathrm{C})$ & 11851 & 2296 & 4463 & 56 \\
\hline $\mathrm{H}(27)$ & 11493 & 3015 & 2572 & 33 \\
\hline $\mathrm{H}(28)$ & 13407 & 2862 & 2549 & 44 \\
\hline $\mathrm{H}(29)$ & 14374 & 4107 & 2218 & 54 \\
\hline $\mathrm{H}(30)$ & 13444 & 5555 & 1966 & 48 \\
\hline $\mathrm{H}(31)$ & 11537 & 5704 & 1970 & 33 \\
\hline $\mathrm{H}(34)$ & 9721 & 3073 & 639 & 23 \\
\hline $\mathrm{H}(35 \mathrm{~A})$ & 11112 & 3614 & 1265 & 27 \\
\hline $\mathrm{H}(35 \mathrm{~B})$ & 10859 & 2703 & 1590 & 27 \\
\hline $\mathrm{H}(36 \mathrm{~A})$ & 12419 & 2325 & 1221 & 30 \\
\hline $\mathrm{H}(36 \mathrm{~B})$ & 11729 & 2567 & 703 & 30 \\
\hline $\mathrm{H}(37)$ & 11332 & 928 & 1293 & 29 \\
\hline $\mathrm{H}(38 \mathrm{~A})$ & 9850 & 479 & 693 & 28 \\
\hline $\mathrm{H}(38 \mathrm{~B})$ & 10083 & 1394 & 368 & 28 \\
\hline H(39A) & 8525 & 1755 & 751 & 27 \\
\hline H(39B) & 9248 & 1537 & 1267 & 27 \\
\hline $\mathrm{H}(40 \mathrm{~A})$ & 11275 & -562 & 568 & 35 \\
\hline $\mathrm{H}(40 \mathrm{~B})$ & 12238 & -582 & 1031 & 35 \\
\hline $\mathrm{H}(42)$ & 13973 & -1329 & 906 & 38 \\
\hline $\mathrm{H}(43)$ & 15311 & -1725 & 425 & 45 \\
\hline $\mathrm{H}(44)$ & 15010 & -1306 & -389 & 45 \\
\hline $\mathrm{H}(45)$ & 13338 & -543 & -718 & 41 \\
\hline H(46) & 12037 & -105 & -231 & 36 \\
\hline $\mathrm{H}(47 \mathrm{~A})$ & 7375 & 4166 & 985 & 26 \\
\hline $\mathrm{H}(47 \mathrm{~B})$ & 7392 & 3038 & 837 & 26 \\
\hline $\mathrm{H}(49)$ & 7354 & 2679 & 20 & 30 \\
\hline $\mathrm{H}(50)$ & 7632 & 3117 & -758 & 36 \\
\hline $\mathrm{H}(51)$ & 8400 & 4627 & -896 & 42 \\
\hline $\mathrm{H}(52)$ & 8868 & 5728 & -263 & 42 \\
\hline
\end{tabular}


S-34

\begin{tabular}{llrrl}
$\mathrm{H}(53)$ & 8587 & 5306 & 515 & 35 \\
$\mathrm{H}(54 \mathrm{~A})$ & 5442 & 4759 & 484 & 53 \\
$\mathrm{H}(54 \mathrm{~B})$ & 4198 & 5151 & 313 & 53 \\
$\mathrm{H}(55 \mathrm{~A})$ & 5073 & 3346 & 77 & 70 \\
$\mathrm{H}(55 \mathrm{~B})$ & 3895 & 3741 & -181 & 70 \\
$\mathrm{H}(56 \mathrm{~A})$ & 3181 & 3564 & 549 & 65 \\
$\mathrm{H}(56 \mathrm{~B})$ & 3583 & 2548 & 346 & 65 \\
$\mathrm{H}(57 \mathrm{~A})$ & 5291 & 2638 & 871 & 66 \\
$\mathrm{H}(57 \mathrm{~B})$ & 4300 & 2755 & 1183 & 66 \\
$\mathrm{H}(57 \mathrm{C})$ & 4976 & 3698 & 1050 & 66 \\
$\mathrm{H}(56 \mathrm{C})$ & 4777 & 3546 & 875 & 81 \\
$\mathrm{H}(56 \mathrm{D})$ & 4140 & 2625 & 608 & 81 \\
$\mathrm{H}(56 \mathrm{E})$ & 3458 & 3601 & 686 & 81 \\
& & & & \\
\hline
\end{tabular}


Table 6. Torsion angles $\left[^{\circ}\right]$ for (trans-16) $)_{2} \bullet$ heptane.

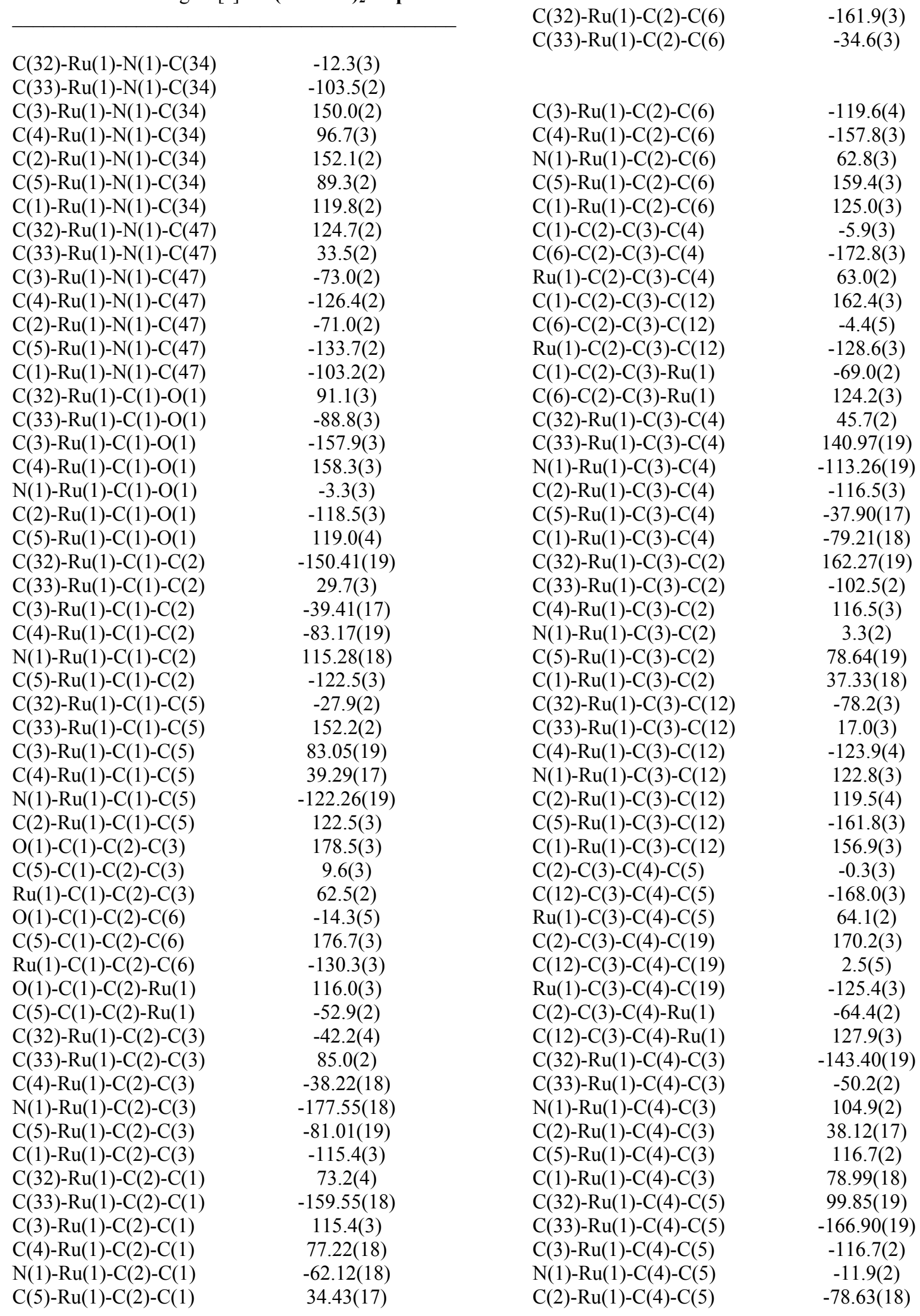




\begin{tabular}{|c|c|}
\hline$C(1)-R u(1)-C(4)-C(5)$ & $-37.75(17)$ \\
\hline$C(32)-R u(1)-C(4)-C(19)$ & $-22.1(3)$ \\
\hline $\mathrm{C}(33)-\mathrm{Ru}(1)-\mathrm{C}(4)-\mathrm{C}(19)$ & $71.1(3)$ \\
\hline$C(3)-R u(1)-C(4)-C(19)$ & $121.3(4)$ \\
\hline $\mathrm{N}(1)-\mathrm{Ru}(1)-\mathrm{C}(4)-\mathrm{C}(19)$ & $-133.9(3)$ \\
\hline$C(2)-R u(1)-C(4)-C(19)$ & $159.4(3)$ \\
\hline $\mathrm{C}(5)-\mathrm{Ru}(1)-\mathrm{C}(4)-\mathrm{C}(19)$ & $-122.0(4)$ \\
\hline $\mathrm{C}(1)-\mathrm{Ru}(1)-\mathrm{C}(4)-\mathrm{C}(19)$ & $-159.7(3)$ \\
\hline $\mathrm{C}(3)-\mathrm{C}(4)-\mathrm{C}(5)-\mathrm{C}(1)$ & $6.3(3)$ \\
\hline $\mathrm{C}(19)-\mathrm{C}(4)-\mathrm{C}(5)-\mathrm{C}(1)$ & $-164.3(3)$ \\
\hline $\mathrm{Ru}(1)-\mathrm{C}(4)-\mathrm{C}(5)-\mathrm{C}(1)$ & $68.7(2)$ \\
\hline$C(3)-C(4)-C(5)-C(26)$ & $174.2(3)$ \\
\hline$C(19)-C(4)-C(5)-C(26)$ & $3.6(5)$ \\
\hline $\mathrm{Ru}(1)-\mathrm{C}(4)-\mathrm{C}(5)-\mathrm{C}(26)$ & $-123.4(3)$ \\
\hline $\mathrm{C}(3)-\mathrm{C}(4)-\mathrm{C}(5)-\mathrm{Ru}(1)$ & $-62.4(2)$ \\
\hline $\mathrm{C}(19)-\mathrm{C}(4)-\mathrm{C}(5)-\mathrm{Ru}(1)$ & $127.0(3)$ \\
\hline $\mathrm{O}(1)-\mathrm{C}(1)-\mathrm{C}(5)-\mathrm{C}(4)$ & $-178.6(3)$ \\
\hline $\mathrm{C}(2)-\mathrm{C}(1)-\mathrm{C}(5)-\mathrm{C}(4)$ & $-9.7(3)$ \\
\hline $\mathrm{Ru}(1)-\mathrm{C}(1)-\mathrm{C}(5)-\mathrm{C}(4)$ & $-62.7(2)$ \\
\hline $\mathrm{O}(1)-\mathrm{C}(1)-\mathrm{C}(5)-\mathrm{C}(26)$ & $12.8(5)$ \\
\hline$C(2)-C(1)-C(5)-C(26)$ & $-178.3(3)$ \\
\hline $\mathrm{Ru}(1)-\mathrm{C}(1)-\mathrm{C}(5)-\mathrm{C}(26)$ & $128.7(3)$ \\
\hline $\mathrm{O}(1)-\mathrm{C}(1)-\mathrm{C}(5)-\mathrm{Ru}(1)$ & $-116.0(3)$ \\
\hline $\mathrm{C}(2)-\mathrm{C}(1)-\mathrm{C}(5)-\mathrm{Ru}(1)$ & $52.9(2)$ \\
\hline$C(32)-R u(1)-C(5)-C(4)$ & $-85.04(19)$ \\
\hline C(33)-Ru(1)-C(5)-C(4) & $34.7(5)$ \\
\hline$C(3)-R u(1)-C(5)-C(4)$ & $38.16(17)$ \\
\hline $\mathrm{N}(1)-\mathrm{Ru}(1)-\mathrm{C}(5)-\mathrm{C}(4)$ & $171.55(17)$ \\
\hline$C(2)-R u(1)-C(5)-C(4)$ & $81.15(19)$ \\
\hline $\mathrm{C}(1)-\mathrm{Ru}(1)-\mathrm{C}(5)-\mathrm{C}(4)$ & $115.2(3)$ \\
\hline $\mathrm{C}(32)-\mathrm{Ru}(1)-\mathrm{C}(5)-\mathrm{C}(1)$ & $159.73(18)$ \\
\hline $\mathrm{C}(33)-\mathrm{Ru}(1)-\mathrm{C}(5)-\mathrm{C}(1)$ & $-80.5(4)$ \\
\hline$C(3)-R u(1)-C(5)-C(1)$ & $-77.08(18)$ \\
\hline$C(4)-R u(1)-C(5)-C(1)$ & $-115.2(3)$ \\
\hline $\mathrm{N}(1)-\mathrm{Ru}(1)-\mathrm{C}(5)-\mathrm{C}(1)$ & $56.31(18)$ \\
\hline$C(2)-R u(1)-C(5)-C(1)$ & $-34.09(17)$ \\
\hline$C(32)-R u(1)-C(5)-C(26)$ & $37.0(3)$ \\
\hline $\mathrm{C}(33)-\mathrm{Ru}(1)-\mathrm{C}(5)-\mathrm{C}(26)$ & $156.8(4)$ \\
\hline$C(3)-R u(1)-C(5)-C(26)$ & $160.2(3)$ \\
\hline $\mathrm{C}(4)-\mathrm{Ru}(1)-\mathrm{C}(5)-\mathrm{C}(26)$ & 122.1(3) \\
\hline $\mathrm{N}(1)-\mathrm{Ru}(1)-\mathrm{C}(5)-\mathrm{C}(26)$ & $-66.4(3)$ \\
\hline $\mathrm{C}(2)-\mathrm{Ru}(1)-\mathrm{C}(5)-\mathrm{C}(26)$ & $-156.8(3)$ \\
\hline $\mathrm{C}(1)-\mathrm{Ru}(1)-\mathrm{C}(5)-\mathrm{C}(26)$ & $-122.7(3)$ \\
\hline$C(3)-C(2)-C(6)-C(7)$ & 130.1(3) \\
\hline$C(1)-C(2)-C(6)-C(7)$ & $-34.9(4)$ \\
\hline $\mathrm{Ru}(1)-\mathrm{C}(2)-\mathrm{C}(6)-\mathrm{C}(7)$ & $-139.1(3)$ \\
\hline$C(3)-C(2)-C(6)-C(11)$ & $-48.6(5)$ \\
\hline$C(1)-C(2)-C(6)-C(11)$ & $146.5(3)$ \\
\hline $\mathrm{Ru}(1)-\mathrm{C}(2)-\mathrm{C}(6)-\mathrm{C}(11)$ & $42.3(4)$ \\
\hline $\mathrm{C}(11)-\mathrm{C}(6)-\mathrm{C}(7)-\mathrm{C}(8)$ & $0.4(5)$ \\
\hline$C(2)-C(6)-C(7)-C(8)$ & $-178.3(3)$ \\
\hline $\mathrm{C}(6)-\mathrm{C}(7)-\mathrm{C}(8)-\mathrm{C}(9)$ & $-1.0(5)$ \\
\hline $\mathrm{C}(7)-\mathrm{C}(8)-\mathrm{C}(9)-\mathrm{C}(10)$ & $0.3(6)$ \\
\hline $\mathrm{C}(8)-\mathrm{C}(9)-\mathrm{C}(10)-\mathrm{C}(11)$ & $1.0(5)$ \\
\hline C(9)-C(10)-C(11)-C(6) & $-1.5(5)$ \\
\hline $\mathrm{C}(7)-\mathrm{C}(6)-\mathrm{C}(11)-\mathrm{C}(10)$ & $0.9(5)$ \\
\hline
\end{tabular}

\begin{tabular}{|c|c|}
\hline$C(2)-C(6)-C(11)-C(10)$ & $179.5(3)$ \\
\hline $\mathrm{C}(4)-\mathrm{C}(3)-\mathrm{C}(12)-\mathrm{C}(17)$ & $-74.5(4)$ \\
\hline$C(2)-C(3)-C(12)-C(17)$ & $119.5(4)$ \\
\hline $\mathrm{Ru}(1)-\mathrm{C}(3)-\mathrm{C}(12)-\mathrm{C}(17)$ & $23.1(5)$ \\
\hline $\mathrm{C}(4)-\mathrm{C}(3)-\mathrm{C}(12)-\mathrm{C}(13)$ & $108.9(4)$ \\
\hline $\mathrm{C}(2)-\mathrm{C}(3)-\mathrm{C}(12)-\mathrm{C}(13)$ & $-57.1(4)$ \\
\hline $\mathrm{Ru}(1)-\mathrm{C}(3)-\mathrm{C}(12)-\mathrm{C}(13)$ & $-153.5(2)$ \\
\hline $\mathrm{C}(17)-\mathrm{C}(12)-\mathrm{C}(13)-\mathrm{C}(14)$ & $-2.0(5)$ \\
\hline $\mathrm{C}(3)-\mathrm{C}(12)-\mathrm{C}(13)-\mathrm{C}(14)$ & $174.8(3)$ \\
\hline$C(12)-C(13)-C(14)-C(15)$ & $0.5(5)$ \\
\hline$C(13)-C(14)-C(15)-C(16)$ & $1.2(5)$ \\
\hline $\mathrm{C}(13)-\mathrm{C}(14)-\mathrm{C}(15)-\mathrm{C}(18)$ & $-176.5(3)$ \\
\hline $\mathrm{C}(14)-\mathrm{C}(15)-\mathrm{C}(16)-\mathrm{C}(17)$ & $-1.4(5)$ \\
\hline $\mathrm{C}(18)-\mathrm{C}(15)-\mathrm{C}(16)-\mathrm{C}(17)$ & $176.3(3)$ \\
\hline $\mathrm{C}(13)-\mathrm{C}(12)-\mathrm{C}(17)-\mathrm{C}(16)$ & $1.8(5)$ \\
\hline $\mathrm{C}(3)-\mathrm{C}(12)-\mathrm{C}(17)-\mathrm{C}(16)$ & $-174.8(3)$ \\
\hline$C(15)-C(16)-C(17)-C(12)$ & $-0.1(5)$ \\
\hline$C(3)-C(4)-C(19)-C(20)$ & $59.6(4)$ \\
\hline$C(5)-C(4)-C(19)-C(20)$ & $-131.5(3)$ \\
\hline $\mathrm{Ru}(1)-\mathrm{C}(4)-\mathrm{C}(19)-\mathrm{C}(20)$ & $-34.5(5)$ \\
\hline$C(3)-C(4)-C(19)-C(24)$ & $-119.3(4)$ \\
\hline$C(5)-C(4)-C(19)-C(24)$ & $49.6(4)$ \\
\hline $\mathrm{Ru}(1)-\mathrm{C}(4)-\mathrm{C}(19)-\mathrm{C}(24)$ & $146.7(3)$ \\
\hline $\mathrm{C}(24)-\mathrm{C}(19)-\mathrm{C}(20)-\mathrm{C}(21)$ & $-0.2(5)$ \\
\hline$C(4)-C(19)-C(20)-C(21)$ & $-179.1(3)$ \\
\hline$C(19)-C(20)-C(21)-C(22)$ & $-0.1(5)$ \\
\hline $\mathrm{C}(20)-\mathrm{C}(21)-\mathrm{C}(22)-\mathrm{C}(23)$ & $-0.3(5)$ \\
\hline $\mathrm{C}(20)-\mathrm{C}(21)-\mathrm{C}(22)-\mathrm{C}(25)$ & $-177.3(3)$ \\
\hline $\mathrm{C}(21)-\mathrm{C}(22)-\mathrm{C}(23)-\mathrm{C}(24)$ & $0.9(5)$ \\
\hline $\mathrm{C}(25)-\mathrm{C}(22)-\mathrm{C}(23)-\mathrm{C}(24)$ & $177.9(3)$ \\
\hline $\mathrm{C}(20)-\mathrm{C}(19)-\mathrm{C}(24)-\mathrm{C}(23)$ & $0.8(5)$ \\
\hline $\mathrm{C}(4)-\mathrm{C}(19)-\mathrm{C}(24)-\mathrm{C}(23)$ & $179.7(3)$ \\
\hline$C(22)-C(23)-C(24)-C(19)$ & $-1.2(5)$ \\
\hline$C(4)-C(5)-C(26)-C(31)$ & $-138.3(3)$ \\
\hline $\mathrm{C}(1)-\mathrm{C}(5)-\mathrm{C}(26)-\mathrm{C}(31)$ & $27.9(4)$ \\
\hline $\mathrm{Ru}(1)-\mathrm{C}(5)-\mathrm{C}(26)-\mathrm{C}(31)$ & $129.6(3)$ \\
\hline$C(4)-C(5)-C(26)-C(27)$ & $42.5(5)$ \\
\hline $\mathrm{C}(1)-\mathrm{C}(5)-\mathrm{C}(26)-\mathrm{C}(27)$ & $-151.2(3)$ \\
\hline $\mathrm{Ru}(1)-\mathrm{C}(5)-\mathrm{C}(26)-\mathrm{C}(27)$ & $-49.6(4)$ \\
\hline $\mathrm{C}(31)-\mathrm{C}(26)-\mathrm{C}(27)-\mathrm{C}(28)$ & $-2.4(5)$ \\
\hline $\mathrm{C}(5)-\mathrm{C}(26)-\mathrm{C}(27)-\mathrm{C}(28)$ & $176.7(3)$ \\
\hline $\mathrm{C}(26)-\mathrm{C}(27)-\mathrm{C}(28)-\mathrm{C}(29)$ & $0.3(5)$ \\
\hline $\mathrm{C}(27)-\mathrm{C}(28)-\mathrm{C}(29)-\mathrm{C}(30)$ & $2.5(6)$ \\
\hline $\mathrm{C}(28)-\mathrm{C}(29)-\mathrm{C}(30)-\mathrm{C}(31)$ & $-3.3(6)$ \\
\hline$C(29)-C(30)-C(31)-C(26)$ & $1.2(6)$ \\
\hline $\mathrm{C}(27)-\mathrm{C}(26)-\mathrm{C}(31)-\mathrm{C}(30)$ & $1.7(5)$ \\
\hline$C(5)-C(26)-C(31)-C(30)$ & $-177.5(3)$ \\
\hline $\mathrm{C}(33)-\mathrm{Ru}(1)-\mathrm{C}(32)-\mathrm{O}(2)$ & $-115(3)$ \\
\hline $\mathrm{C}(3)-\mathrm{Ru}(1)-\mathrm{C}(32)-\mathrm{O}(2)$ & $-16(3)$ \\
\hline $\mathrm{C}(4)-\mathrm{Ru}(1)-\mathrm{C}(32)-\mathrm{O}(2)$ & $10(3)$ \\
\hline $\mathrm{N}(1)-\mathrm{Ru}(1)-\mathrm{C}(32)-\mathrm{O}(2)$ & $148(3)$ \\
\hline $\mathrm{C}(2)-\mathrm{Ru}(1)-\mathrm{C}(32)-\mathrm{O}(2)$ & $14(4)$ \\
\hline $\mathrm{C}(5)-\mathrm{Ru}(1)-\mathrm{C}(32)-\mathrm{O}(2)$ & $48(3)$ \\
\hline $\mathrm{C}(1)-\mathrm{Ru}(1)-\mathrm{C}(32)-\mathrm{O}(2)$ & $65(3)$ \\
\hline $\mathrm{C}(32)-\mathrm{Ru}(1)-\mathrm{C}(33)-\mathrm{O}(3)$ & $88(6)$ \\
\hline $\mathrm{C}(3)-\mathrm{Ru}(1)-\mathrm{C}(33)-\mathrm{O}(3)$ & $-36(6)$ \\
\hline
\end{tabular}




$\begin{array}{lclc}\mathrm{C}(4)-\mathrm{Ru}(1)-\mathrm{C}(33)-\mathrm{O}(3) & -8(6) & \mathrm{C}(46)-\mathrm{C}(41)-\mathrm{C}(42)-\mathrm{C}(43) & 1.0(6) \\ \mathrm{N}(1)-\mathrm{Ru}(1)-\mathrm{C}(33)-\mathrm{O}(3) & -170(6) & \mathrm{C}(40)-\mathrm{C}(41)-\mathrm{C}(42)-\mathrm{C}(43) & -174.9(4) \\ \mathrm{C}(2)-\mathrm{Ru}(1)-\mathrm{C}(33)-\mathrm{O}(3) & -75(6) & \mathrm{C}(41)-\mathrm{C}(42)-\mathrm{C}(43)-\mathrm{C}(44) & -0.5(6) \\ \mathrm{C}(5)-\mathrm{Ru}(1)-\mathrm{C}(33)-\mathrm{O}(3) & -33(6) & \mathrm{C}(42)-\mathrm{C}(43)-\mathrm{C}(44)-\mathrm{C}(45) & -1.1(6) \\ \mathrm{C}(1)-\mathrm{Ru}(1)-\mathrm{C}(33)-\mathrm{O}(3) & -92(6) & \mathrm{C}(43)-\mathrm{C}(44)-\mathrm{C}(45)-\mathrm{C}(46) & 2.2(6) \\ \mathrm{C}(47)-\mathrm{N}(1)-\mathrm{C}(34)-\mathrm{C}(39) & -68.3(3) & \mathrm{C}(44)-\mathrm{C}(45)-\mathrm{C}(46)-\mathrm{C}(41) & -1.7(6) \\ \mathrm{Ru}(1)-\mathrm{N}(1)-\mathrm{C}(34)-\mathrm{C}(39) & 68.9(3) & \mathrm{C}(42)-\mathrm{C}(41)-\mathrm{C}(46)-\mathrm{C}(45) & 0.1(6) \\ \mathrm{C}(47)-\mathrm{N}(1)-\mathrm{C}(34)-\mathrm{C}(35) & 166.5(3) & \mathrm{C}(40)-\mathrm{C}(41)-\mathrm{C}(46)-\mathrm{C}(45) & 176.2(3) \\ \mathrm{Ru}(1)-\mathrm{N}(1)-\mathrm{C}(34)-\mathrm{C}(35) & -56.4(3) & \mathrm{C}(34)-\mathrm{N}(1)-\mathrm{C}(47)-\mathrm{C}(48) & -52.4(4) \\ \mathrm{N}(1)-\mathrm{C}(34)-\mathrm{C}(35)-\mathrm{C}(36) & -175.4(3) & \mathrm{Ru}(1)-\mathrm{N}(1)-\mathrm{C}(47)-\mathrm{C}(48) & 166.6(2) \\ \mathrm{C}(39)-\mathrm{C}(34)-\mathrm{C}(35)-\mathrm{C}(36) & 57.8(3) & \mathrm{N}(1)-\mathrm{C}(47)-\mathrm{C}(48)-\mathrm{C}(53) & -66.8(4) \\ \mathrm{C}(34)-\mathrm{C}(35)-\mathrm{C}(36)-\mathrm{C}(37) & -55.7(4) & \mathrm{N}(1)-\mathrm{C}(47)-\mathrm{C}(48)-\mathrm{C}(49) & 111.2(4) \\ \mathrm{C}(40)-\mathrm{N}(2)-\mathrm{C}(37)-\mathrm{C}(38) & -73.5(4) & \mathrm{C}(53)-\mathrm{C}(48)-\mathrm{C}(49)-\mathrm{C}(50) & 0.5(5) \\ \mathrm{C}(40)-\mathrm{N}(2)-\mathrm{C}(37)-\mathrm{C}(36) & 166.2(3) & \mathrm{C}(47)-\mathrm{C}(48)-\mathrm{C}(49)-\mathrm{C}(50) & -177.5(3) \\ \mathrm{C}(35)-\mathrm{C}(36)-\mathrm{C}(37)-\mathrm{N}(2) & 174.4(3) & \mathrm{C}(48)-\mathrm{C}(49)-\mathrm{C}(50)-\mathrm{C}(51) & 0.2(6) \\ \mathrm{C}(35)-\mathrm{C}(36)-\mathrm{C}(37)-\mathrm{C}(38) & 54.1(4) & \mathrm{C}(49)-\mathrm{C}(50)-\mathrm{C}(51)-\mathrm{C}(52) & -0.7(6) \\ \mathrm{N}(2)-\mathrm{C}(37)-\mathrm{C}(38)-\mathrm{C}(39) & -172.5(3) & \mathrm{C}(50)-\mathrm{C}(51)-\mathrm{C}(52)-\mathrm{C}(53) & 0.5(6) \\ \mathrm{C}(36)-\mathrm{C}(37)-\mathrm{C}(38)-\mathrm{C}(39) & -54.7(4) & \mathrm{C}(49)-\mathrm{C}(48)-\mathrm{C}(53)-\mathrm{C}(52) & -0.7(5) \\ \mathrm{N}(1)-\mathrm{C}(34)-\mathrm{C}(39)-\mathrm{C}(38) & 176.9(3) & \mathrm{C}(47)-\mathrm{C}(48)-\mathrm{C}(53)-\mathrm{C}(52) & 177.3(3) \\ \mathrm{C}(35)-\mathrm{C}(34)-\mathrm{C}(39)-\mathrm{C}(38) & -58.2(3) & \mathrm{C}(51)-\mathrm{C}(52)-\mathrm{C}(53)-\mathrm{C}(48) & 0.2(6) \\ \mathrm{C}(37)-\mathrm{C}(38)-\mathrm{C}(39)-\mathrm{C}(34) & 57.0(4) & \mathrm{C}(54) \# 1-\mathrm{C}(54)-\mathrm{C}(55)-\mathrm{C}(56) & -170.3(7) \\ \mathrm{C}(37)-\mathrm{N}(2)-\mathrm{C}(40)-\mathrm{C}(41) & 178.1(3) & \mathrm{C}(54) \# 1-\mathrm{C}(54)-\mathrm{C}(55)-\mathrm{C}(56 \mathrm{~A}) & 174.6(7) \\ \mathrm{N}(2)-\mathrm{C}(40)-\mathrm{C}(41)-\mathrm{C}(42) & 112.0(4) & \mathrm{C}(54)-\mathrm{C}(55)-\mathrm{C}(56)-\mathrm{C}(57) & -64.6(13) \\ \mathrm{N}(2)-\mathrm{C}(40)-\mathrm{C}(41)-\mathrm{C}(46) & -63.8(4) & \mathrm{C}(56 \mathrm{~A})-\mathrm{C}(55)-\mathrm{C}(56)-\mathrm{C}(57) & -20(3) \\ \end{array}$

Symmetry transformations used to generate equivalent atoms:

$\# 1-\mathrm{x}+1,-\mathrm{y}+1,-\mathrm{z}$

Table 7. Hydrogen bonds for (trans-16) $)_{2} \bullet$ heptane $\left[\AA\right.$ and $\left.{ }^{\circ}\right]$.

\begin{tabular}{lcccc}
\hline $\mathrm{D}-\mathrm{H} \ldots \mathrm{A}$ & $\mathrm{d}(\mathrm{D}-\mathrm{H})$ & $\mathrm{d}(\mathrm{H} \ldots \mathrm{A})$ & $\mathrm{d}(\mathrm{D} \ldots \mathrm{A})$ & $<(\mathrm{DHA})$ \\
\hline $\mathrm{N}(1)-\mathrm{H}(1) \ldots \mathrm{O}(1)$ & $0.88(3)$ & $2.10(3)$ & $2.882(4)$ & $147(3)$
\end{tabular}

Symmetry transformations used to generate equivalent atoms: $\# 1-\mathrm{x}+1,-\mathrm{y}+1,-\mathrm{z}$ 
X-ray Crystal Structure of $23 \bullet\left(\mathrm{CH}_{2} \mathbf{C l}_{2}\right)_{2}$. Data Collection. A pale yellow crystal with approximate dimensions $0.46 \times 0.20 \times 0.04 \mathrm{~mm}$ was selected under oil under ambient conditions and attached to the tip of a nylon loop. The crystal was mounted in a stream of cold nitrogen at 100(2) $\mathrm{K}$ and centered in the X-ray beam by using a video camera. The crystal evaluation and data collection were performed on a Bruker CCD-1000 diffractometer with $\mathrm{Mo} \mathrm{K}_{\alpha}(\lambda=0.71073$ Å) radiation and the diffractometer to crystal distance of $4.9 \mathrm{~cm}$. The initial cell constants were obtained from three series of $\omega$ scans at different starting angles. Each series consisted of 20 frames collected at intervals of $0.3^{\circ}$ in a $6^{\circ}$ range about $\omega$ with the exposure time of 10 seconds per frame. A total of 73 reflections were obtained. The reflections were successfully indexed by an automated indexing routine built in the SMART program. The final cell constants were calculated from a set of 6267 strong reflections from the actual data collection. The data were collected by using the full sphere data collection routine to survey the reciprocal space to the extent of a full sphere to a resolution of $0.80 \AA$. A total of 37090 data were harvested by collecting four sets of frames with $0.36^{\circ}$ scans in $\omega$ with an exposure time 44 sec per frame and one set of frames with $0.45^{\circ}$ scans in $\varphi$ with an exposure time $44 \mathrm{sec}$ per frame. These highly redundant datasets were corrected for Lorentz and polarization effects. The absorption correction was based on fitting a function to the empirical transmission surface as sampled by multiple equivalent measurements. [1]

Structure Solution and Refinement. The systematic absences in the diffraction data were consistent for the space groups $P \overline{1}$ and $P 1$. The $E$-statistics strongly suggested the centrosymmetric space group $P \overline{1}$ that yielded chemically reasonable and computationally stable results of refinement [1]. A successful solution by the direct methods provided most nonhydrogen atoms from the E-map. The remaining non-hydrogen atoms were located in an 
alternating series of least-squares cycles and difference Fourier maps. All non-hydrogen atoms were refined with anisotropic displacement coefficients. All hydrogen atoms were included in the structure factor calculation at idealized positions and were allowed to ride on the neighboring atoms with relative isotropic displacement coefficients. The dimer resides on the inversion center. There are 4 molecules of solvate dichloromethane and two molecules of either toluene or benzene solvate per dimer. Therefore there are two molecules of solvate dichloromethane and one molecule of either toluene or benzene in the asymmetric unit. One of these dichloromethane molecules is well behaved and was refined. One solvate dichloromethane molecule and one solvate toluene or benzene molecule were disordered over several positions. A significant amount of time was invested in identifying and refining the disordered molecules. Bond length restraints were applied to model the molecules but the resulting isotropic displacement coefficients suggested the molecules were mobile. In addition, the refinement was computationally unstable. Option SQUEEZE of program PLATON [2] was used to correct the diffraction data for diffuse scattering effects and to identify the solvate molecule. PLATON calculated the upper limit of volume that can be occupied by the solvent to be $462.9 \AA^{3}$, or $21.1 \%$ of the unit cell volume. The program calculated 91 electrons in the unit cell for the diffuse species. This approximately corresponds to two molecules of dichloromethane and two molecules of toluene or benzene per dimer (100 or 84 electrons). It was not possible to identify whether the solvent was toluene or benzene but since the crystallization was performed in toluene, the composition of the complex reflects two dichloromethane molecules and one toluene molecule in the asymmetric unit. There is one intramolecular hydrogen bond in the asymmetric unit between $\mathrm{N} 1$ and $\mathrm{O} 1$. 
The final least-squares refinement of 453 parameters against 10795 data resulted in residuals $R$ (based on $F^{2}$ for $I \geq 2 \sigma$ ) and $w R$ (based on $F^{2}$ for all data) of 0.0358 and 0.0897 , respectively. There was a peak of $1.147 \mathrm{e} . \AA^{-3}$ near the ruthenium atom which was determined to be noise. The molecular diagram is drawn with $30 \%$ probability ellipsoids.

\section{References}

[1] Bruker-AXS. (2000-2003) SADABS V.2.05, SAINT V.6.22, SHELXTL V.6.10

\& SMART 5.622 Software Reference Manuals. Bruker-AXS, Madison, Wisconsin, USA.

[2] A.L. Spek (1990) Acta Cryst. A46, C34. 

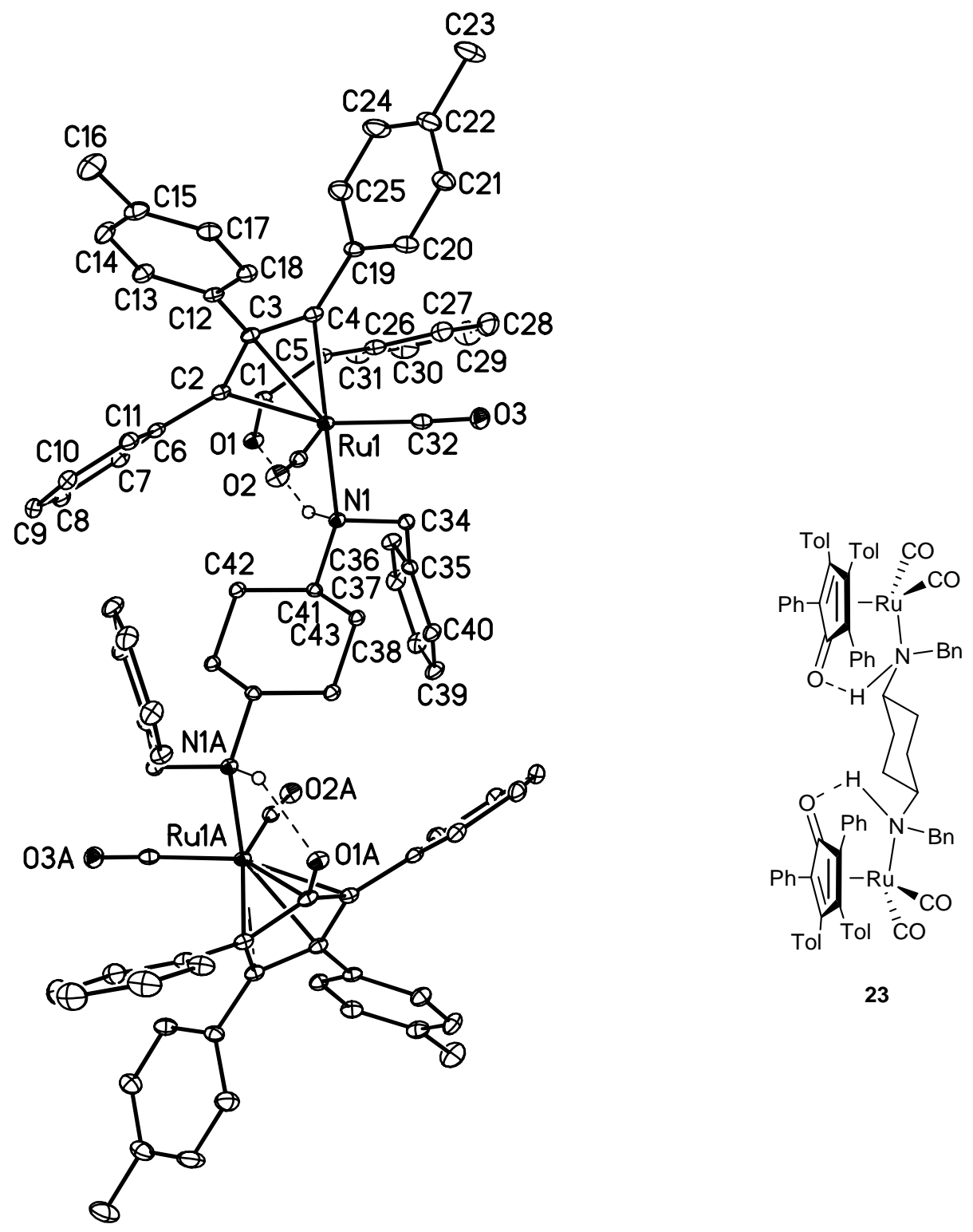

Figure S-4. The structure of $\mathbf{2 3} \bullet\left(\mathbf{C H}_{\mathbf{2}} \mathbf{C l}_{\mathbf{2}}\right)_{2}$ drawn with $30 \%$ probability ellipsoids. All hydrogen atoms except the amine hydrogen atoms were omitted for clarity. The intramolecular hydrogen bonds are shown with dashed lines. 


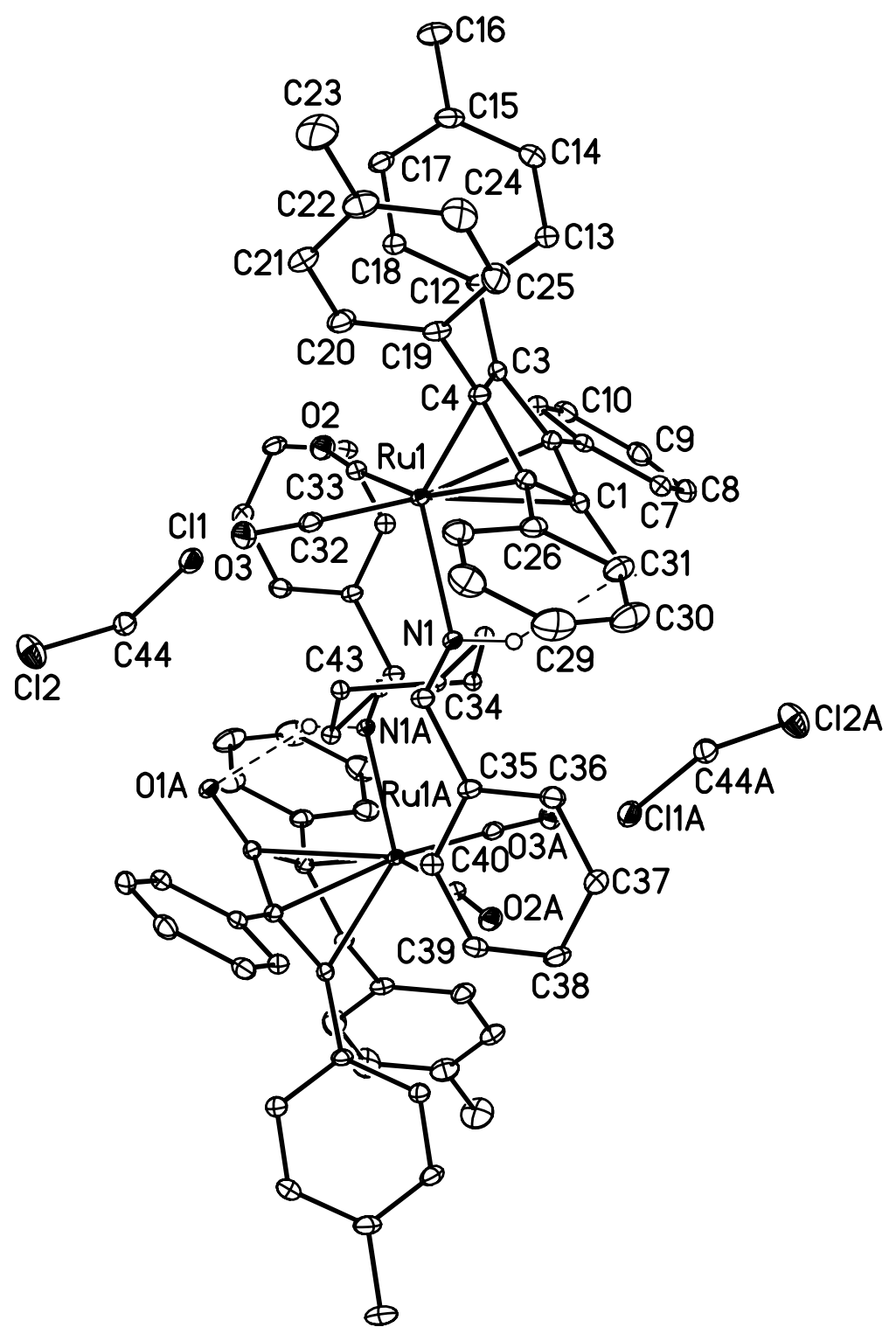

Figure S-5. The structure of the $\mathbf{2 3} \bullet\left(\mathbf{C H}_{\mathbf{2}} \mathbf{C} \mathbf{C l}_{2}\right)_{2}$ and the two ordered dichloromethane solvent molecules drawn with $30 \%$ probability ellipsoids. All hydrogen atoms except the amine hydrogen atoms were omitted for clarity. The intramolecular hydrogen bonds are shown with dashed lines. 
Table 1. Crystal data and structure refinement for $\mathbf{2 3} \bullet\left(\mathbf{C H}_{\mathbf{2}} \mathbf{C l}_{\mathbf{2}}\right)_{\mathbf{2}}$.

Empirical formula

Formula weight

Temperature

Wavelength

Crystal system

Space group

Unit cell dimensions

Volume

Z

Density (calculated)

Absorption coefficient

$\mathrm{F}(000)$

Crystal size

Theta range for data collection

Index ranges

Reflections collected

Independent reflections

Completeness to theta $=28.34^{\circ}$

Absorption correction

Max. and min. transmission

Refinement method

Data / restraints / parameters

Goodness-of-fit on $\mathrm{F}^{2}$

Final $\mathrm{R}$ indices $[\mathrm{I}>2 \operatorname{sigma}(\mathrm{I})]$

$\mathrm{R}$ indices (all data)

Largest diff. peak and hole
$\mathrm{C}_{86} \mathrm{H}_{74} \mathrm{~N}_{2} \mathrm{O}_{6} \mathrm{Ru}_{2} \cdot 4 \mathrm{CH}_{2} \mathrm{Cl}_{2} \cdot 2 \mathrm{C}_{7} \mathrm{H}_{8}$

1955.57

100(2) K

$0.71073 \AA$

Triclinic

$\mathrm{P} 1$

$\mathrm{a}=9.898(2) \AA \quad \alpha=80.245(3)^{\circ}$.

$\mathrm{b}=10.507(2) \AA \quad \beta=87.922(4)^{\circ}$.

$\mathrm{c}=21.638(4) \AA \quad \gamma=81.829(3)^{\circ}$.

2195.1(8) $\AA^{3}$

1

$1.479 \mathrm{Mg} / \mathrm{m}^{3}$

$0.647 \mathrm{~mm}^{-1}$

1006

$0.46 \times 0.20 \times 0.04 \mathrm{~mm}^{3}$

0.95 to $28.34^{\circ}$.

$-13<=\mathrm{h}<=13,-13<=\mathrm{k}<=14,-28<=\mathrm{l}<=28$

37090

$10795[\mathrm{R}(\mathrm{int})=0.0391]$

$98.7 \%$

Multi-scan with SADABS

0.9746 and 0.7552

Full-matrix least-squares on $\mathrm{F}^{2}$

10795 / 0 / 453

0.854

$\mathrm{R} 1=0.0358, \mathrm{wR} 2=0.0858$

$\mathrm{R} 1=0.0445, \mathrm{wR} 2=0.0897$

1.147 and -0.338 e. $\AA^{-3}$ 
Table 2. Atomic coordinates $\left(\times 10^{4}\right)$ and equivalent isotropic displacement parameters $\left(\AA^{2} \times 10^{3}\right)$

for $\mathbf{2 3} \bullet\left(\mathbf{C H}_{2} \mathbf{C l}_{2}\right)_{2}$. U(eq) is defined as one third of the trace of the orthogonalized $\mathrm{U}^{\mathrm{ij}}$ tensor.

\begin{tabular}{|c|c|c|c|c|}
\hline & $\mathrm{x}$ & $\mathrm{y}$ & $\mathrm{z}$ & $\mathrm{U}(\mathrm{eq})$ \\
\hline $\mathrm{Ru}(1)$ & 283(1) & $9355(1)$ & $8117(1)$ & $14(1)$ \\
\hline $\mathrm{O}(1)$ & $1893(2)$ & $11944(1)$ & 7903(1) & $20(1)$ \\
\hline $\mathrm{O}(2)$ & $677(2)$ & $6935(1)$ & 9112(1) & $25(1)$ \\
\hline $\mathrm{O}(3)$ & $-2640(2)$ & $8968(2)$ & 7899(1) & $28(1)$ \\
\hline $\mathrm{N}(1)$ & $-301(2)$ & $10965(2)$ & $8669(1)$ & $14(1)$ \\
\hline$C(1)$ & $1826(2)$ & $10902(2)$ & 7703(1) & $17(1)$ \\
\hline$C(2)$ & $2507(2)$ & $9586(2)$ & $7968(1)$ & $17(1)$ \\
\hline$C(3)$ & $2130(2)$ & $8679(2)$ & $7588(1)$ & $17(1)$ \\
\hline C(4) & 1099(2) & 9361(2) & 7158(1) & $18(1)$ \\
\hline$C(5)$ & $839(2)$ & $10705(2)$ & $7247(1)$ & $17(1)$ \\
\hline$C(6)$ & $3522(2)$ & $9307(2)$ & 8471(1) & $18(1)$ \\
\hline$C(7)$ & $4224(2)$ & $10299(2)$ & $8603(1)$ & $21(1)$ \\
\hline $\mathrm{C}(8)$ & $5139(2)$ & $10053(2)$ & 9096(1) & $24(1)$ \\
\hline$C(9)$ & $5403(2)$ & $8830(2)$ & $9458(1)$ & $24(1)$ \\
\hline$C(10)$ & $4725(2)$ & $7837(2)$ & $9327(1)$ & $22(1)$ \\
\hline $\mathrm{C}(11)$ & $3799(2)$ & 8071(2) & $8842(1)$ & $20(1)$ \\
\hline$C(12)$ & $2867(2)$ & $7355(2)$ & 7559(1) & $18(1)$ \\
\hline$C(13)$ & $4296(2)$ & $7186(2)$ & $7516(1)$ & $23(1)$ \\
\hline$C(14)$ & $5024(2)$ & $5972(2)$ & 7476(1) & $28(1)$ \\
\hline$C(15)$ & 4359(2) & $4890(2)$ & 7475(1) & $26(1)$ \\
\hline$C(16)$ & $5167(3)$ & $3568(2)$ & $7460(1)$ & $39(1)$ \\
\hline$C(17)$ & 2943(2) & $5061(2)$ & $7506(1)$ & $23(1)$ \\
\hline$C(18)$ & $2202(2)$ & $6275(2)$ & 7550(1) & $20(1)$ \\
\hline $\mathrm{C}(19)$ & 610(2) & $8841(2)$ & 6616(1) & $19(1)$ \\
\hline$C(20)$ & $-433(2)$ & $8066(2)$ & $6663(1)$ & $25(1)$ \\
\hline$C(21)$ & $-850(2)$ & 7631(2) & $6140(1)$ & $26(1)$ \\
\hline$C(22)$ & $-241(3)$ & $7935(2)$ & 5559(1) & $31(1)$ \\
\hline$C(23)$ & $-694(3)$ & 7438(3) & 4995(1) & $46(1)$ \\
\hline$C(24)$ & $802(3)$ & 8709(3) & $5515(1)$ & $38(1)$ \\
\hline$C(25)$ & $1222(3)$ & $9156(2)$ & $6035(1)$ & $30(1)$ \\
\hline$C(26)$ & $-47(2)$ & $11776(2)$ & 6862(1) & $21(1)$ \\
\hline$C(27)$ & $-1307(2)$ & $11605(2)$ & $6639(1)$ & $27(1)$ \\
\hline$C(28)$ & $-2116(3)$ & $12633(3)$ & $6278(1)$ & $37(1)$ \\
\hline$C(29)$ & $-1689(3)$ & $13848(3)$ & $6140(1)$ & $42(1)$ \\
\hline $\mathrm{C}(30)$ & $-443(3)$ & $14028(2)$ & 6351(1) & $39(1)$ \\
\hline$C(31)$ & $376(3)$ & $13007(2)$ & 6709(1) & $27(1)$ \\
\hline$C(32)$ & $-1552(2)$ & $9124(2)$ & 7989(1) & 19(1) \\
\hline$C(33)$ & 471(2) & 7874(2) & $8756(1)$ & $17(1)$ \\
\hline $\mathrm{C}(34)$ & $-1604(2)$ & 11791(2) & $8417(1)$ & $17(1)$ \\
\hline$C(35)$ & $-1826(2)$ & $13145(2)$ & $8587(1)$ & $17(1)$ \\
\hline$C(36)$ & $-1259(2)$ & $14145(2)$ & $8206(1)$ & $22(1)$ \\
\hline$C(37)$ & $-1482(2)$ & $15404(2)$ & 8344(1) & $25(1)$ \\
\hline $\mathrm{C}(38)$ & $-2251(2)$ & $15677(2)$ & 8862(1) & $26(1)$ \\
\hline C(39) & $-2807(2)$ & $14684(2)$ & 9249(1) & $25(1)$ \\
\hline$C(40)$ & $-2606(2)$ & $13425(2)$ & 9106(1) & $21(1)$ \\
\hline $\mathrm{C}(41)$ & $-249(2)$ & $10763(2)$ & 9371(1) & $14(1)$ \\
\hline $\mathrm{C}(42)$ & 1196(2) & $10226(2)$ & 9596(1) & $16(1)$ \\
\hline$C(43)$ & $-1274(1)$ & $9897(1)$ & $9690(1)$ & $15(1)$ \\
\hline $\mathrm{Cl}(1)$ & $8849(1)$ & 6359(1) & $332(1)$ & $28(1)$ \\
\hline
\end{tabular}


S-45

$\mathrm{Cl}(2)$

C(44)
6159(1)

7888(1)
6001(1)

6080(1)
886(1)

1035(1)
40(1)

24(1) 
Table 3. Bond lengths $[\AA]$ and angles $\left[^{\circ}\right]$ for $\mathbf{2 3} \mathbf{\bullet}\left(\mathbf{C H}_{\mathbf{2}} \mathbf{C l}_{\mathbf{2}}\right)_{\mathbf{2}}$.

\begin{tabular}{|c|c|c|c|}
\hline $\mathrm{Ru}(1)-\mathrm{C}(33)$ & $1.891(2)$ & $C(20)-C(21)$ & $1.388(3)$ \\
\hline $\mathrm{Ru}(1)-\mathrm{C}(32)$ & $1.903(2)$ & $\mathrm{C}(20)-\mathrm{H}(20)$ & 0.9500 \\
\hline $\mathrm{Ru}(1)-\mathrm{C}(4)$ & $2.199(2)$ & $\mathrm{C}(21)-\mathrm{C}(22)$ & $1.381(3)$ \\
\hline $\mathrm{Ru}(1)-\mathrm{C}(3)$ & $2.2158(19)$ & $\mathrm{C}(21)-\mathrm{H}(21)$ & 0.9500 \\
\hline $\mathrm{Ru}(1)-\mathrm{N}(1)$ & $2.2306(15)$ & $\mathrm{C}(22)-\mathrm{C}(24)$ & $1.393(4)$ \\
\hline $\mathrm{Ru}(1)-\mathrm{C}(2)$ & $2.254(2)$ & $\mathrm{C}(22)-\mathrm{C}(23)$ & $1.512(3)$ \\
\hline $\mathrm{Ru}(1)-\mathrm{C}(5)$ & $2.258(2)$ & $\mathrm{C}(23)-\mathrm{H}(23 \mathrm{~A})$ & 0.9800 \\
\hline $\mathrm{Ru}(1)-\mathrm{C}(1)$ & $2.4266(19)$ & $\mathrm{C}(23)-\mathrm{H}(23 \mathrm{~B})$ & 0.9800 \\
\hline $\mathrm{Ru}(1)-\operatorname{cent}(\mathrm{C} 1-\mathrm{C} 5)$ & $1.906(2)$ & $\mathrm{C}(23)-\mathrm{H}(23 \mathrm{C})$ & 0.9800 \\
\hline $\mathrm{O}(1)-\mathrm{C}(1)$ & $1.256(2)$ & $\mathrm{C}(24)-\mathrm{C}(25)$ & $1.389(3)$ \\
\hline $\mathrm{O}(2)-\mathrm{C}(33)$ & $1.142(2)$ & $\mathrm{C}(24)-\mathrm{H}(24)$ & 0.9500 \\
\hline $\mathrm{O}(3)-\mathrm{C}(32)$ & $1.140(3)$ & $\mathrm{C}(25)-\mathrm{H}(25)$ & 0.9500 \\
\hline $\mathrm{N}(1)-\mathrm{C}(41)$ & $1.500(2)$ & $\mathrm{C}(26)-\mathrm{C}(31)$ & $1.399(3)$ \\
\hline $\mathrm{N}(1)-\mathrm{C}(34)$ & $1.511(2)$ & $\mathrm{C}(26)-\mathrm{C}(27)$ & $1.400(3)$ \\
\hline $\mathrm{N}(1)-\mathrm{H}(1)$ & 0.9300 & $\mathrm{C}(27)-\mathrm{C}(28)$ & $1.390(3)$ \\
\hline$C(1)-C(5)$ & $1.471(3)$ & $\mathrm{C}(27)-\mathrm{H}(27)$ & 0.9500 \\
\hline $\mathrm{C}(1)-\mathrm{C}(2)$ & $1.479(3)$ & $\mathrm{C}(28)-\mathrm{C}(29)$ & $1.383(4)$ \\
\hline$C(2)-C(3)$ & $1.452(3)$ & $\mathrm{C}(28)-\mathrm{H}(28)$ & 0.9500 \\
\hline$C(2)-C(6)$ & $1.472(3)$ & $\mathrm{C}(29)-\mathrm{C}(30)$ & $1.380(4)$ \\
\hline$C(3)-C(4)$ & $1.435(3)$ & $\mathrm{C}(29)-\mathrm{H}(29)$ & 0.9500 \\
\hline $\mathrm{C}(3)-\mathrm{C}(12)$ & $1.486(3)$ & $\mathrm{C}(30)-\mathrm{C}(31)$ & $1.387(3)$ \\
\hline$C(4)-C(5)$ & $1.445(3)$ & $\mathrm{C}(30)-\mathrm{H}(30)$ & 0.9500 \\
\hline $\mathrm{C}(4)-\mathrm{C}(19)$ & $1.495(3)$ & $\mathrm{C}(31)-\mathrm{H}(31)$ & 0.9500 \\
\hline$C(5)-C(26)$ & $1.478(3)$ & $\mathrm{C}(34)-\mathrm{C}(35)$ & $1.513(3)$ \\
\hline $\mathrm{C}(6)-\mathrm{C}(11)$ & $1.403(3)$ & $\mathrm{C}(34)-\mathrm{H}(34 \mathrm{~A})$ & 0.9900 \\
\hline $\mathrm{C}(6)-\mathrm{C}(7)$ & $1.404(3)$ & $\mathrm{C}(34)-\mathrm{H}(34 \mathrm{~B})$ & 0.9900 \\
\hline$C(7)-C(8)$ & $1.392(3)$ & $\mathrm{C}(35)-\mathrm{C}(40)$ & $1.389(3)$ \\
\hline $\mathrm{C}(7)-\mathrm{H}(7)$ & 0.9500 & $\mathrm{C}(35)-\mathrm{C}(36)$ & $1.392(3)$ \\
\hline $\mathrm{C}(8)-\mathrm{C}(9)$ & $1.382(3)$ & $\mathrm{C}(36)-\mathrm{C}(37)$ & $1.391(3)$ \\
\hline $\mathrm{C}(8)-\mathrm{H}(8)$ & 0.9500 & $\mathrm{C}(36)-\mathrm{H}(36)$ & 0.9500 \\
\hline $\mathrm{C}(9)-\mathrm{C}(10)$ & $1.391(3)$ & $\mathrm{C}(37)-\mathrm{C}(38)$ & $1.379(3)$ \\
\hline $\mathrm{C}(9)-\mathrm{H}(9)$ & 0.9500 & $\mathrm{C}(37)-\mathrm{H}(37)$ & 0.9500 \\
\hline $\mathrm{C}(10)-\mathrm{C}(11)$ & $1.385(3)$ & $\mathrm{C}(38)-\mathrm{C}(39)$ & $1.389(3)$ \\
\hline $\mathrm{C}(10)-\mathrm{H}(10)$ & 0.9500 & $\mathrm{C}(38)-\mathrm{H}(38)$ & 0.9500 \\
\hline $\mathrm{C}(11)-\mathrm{H}(11)$ & 0.9500 & $C(39)-C(40)$ & $1.394(3)$ \\
\hline $\mathrm{C}(12)-\mathrm{C}(18)$ & $1.394(3)$ & $\mathrm{C}(39)-\mathrm{H}(39)$ & 0.9500 \\
\hline$C(12)-C(13)$ & $1.402(3)$ & $\mathrm{C}(40)-\mathrm{H}(40)$ & 0.9500 \\
\hline$C(13)-C(14)$ & $1.388(3)$ & $\mathrm{C}(41)-\mathrm{C}(42)$ & $1.526(3)$ \\
\hline $\mathrm{C}(13)-\mathrm{H}(13)$ & 0.9500 & $\mathrm{C}(41)-\mathrm{C}(43)$ & $1.5279(19)$ \\
\hline$C(14)-C(15)$ & $1.393(3)$ & $\mathrm{C}(41)-\mathrm{H}(41)$ & 1.0000 \\
\hline $\mathrm{C}(14)-\mathrm{H}(14)$ & 0.9500 & $C(42)-C(43) \# 1$ & $1.532(2)$ \\
\hline$C(15)-C(17)$ & $1.388(3)$ & $\mathrm{C}(42)-\mathrm{H}(42 \mathrm{~A})$ & 0.9900 \\
\hline$C(15)-C(16)$ & $1.506(3)$ & $\mathrm{C}(42)-\mathrm{H}(42 \mathrm{~B})$ & 0.9900 \\
\hline $\mathrm{C}(16)-\mathrm{H}(16 \mathrm{~A})$ & 0.9800 & $\mathrm{C}(43)-\mathrm{C}(42) \# 1$ & $1.532(2)$ \\
\hline $\mathrm{C}(16)-\mathrm{H}(16 \mathrm{~B})$ & 0.9800 & $\mathrm{C}(43)-\mathrm{H}(43 \mathrm{~A})$ & 0.9900 \\
\hline$C(16)-H(16 C)$ & 0.9800 & $\mathrm{C}(43)-\mathrm{H}(43 \mathrm{~B})$ & 0.9900 \\
\hline $\mathrm{C}(17)-\mathrm{C}(18)$ & $1.394(3)$ & $\mathrm{Cl}(1)-\mathrm{C}(44)$ & 1.7676 \\
\hline $\mathrm{C}(17)-\mathrm{H}(17)$ & 0.9500 & $\mathrm{Cl}(2)-\mathrm{C}(44)$ & 1.7686 \\
\hline $\mathrm{C}(18)-\mathrm{H}(18)$ & 0.9500 & $\mathrm{C}(44)-\mathrm{H}(44 \mathrm{~A})$ & 0.9900 \\
\hline$C(19)-C(25)$ & $1.386(3)$ & $\mathrm{C}(44)-\mathrm{H}(44 \mathrm{~B})$ & 0.9900 \\
\hline$C(19)-C(20)$ & $1.394(3)$ & & \\
\hline $\mathrm{C}(33)-\mathrm{Ru}(1)-\mathrm{C}(32)$ & $90.38(9)$ & $\mathrm{C}(33)-\mathrm{Ru}(1)-\mathrm{C}(4)$ & $124.14(8)$ \\
\hline
\end{tabular}




\begin{tabular}{|c|c|c|c|}
\hline$C(32)-R u(1)-C(4)$ & $99.78(8)$ & $C(4)-C(5)-C(1)$ & $108.11(17)$ \\
\hline$C(33)-R u(1)-C(3)$ & $94.68(8)$ & $C(4)-C(5)-C(26)$ & $126.64(18)$ \\
\hline $\mathrm{C}(32)-\mathrm{Ru}(1)-\mathrm{C}(3)$ & $127.65(8)$ & $\mathrm{C}(1)-\mathrm{C}(5)-\mathrm{C}(26)$ & $124.10(17)$ \\
\hline $\mathrm{C}(4)-\mathrm{Ru}(1)-\mathrm{C}(3)$ & $37.92(7)$ & $\mathrm{C}(4)-\mathrm{C}(5)-\mathrm{Ru}(1)$ & $68.90(11)$ \\
\hline $\mathrm{C}(33)-\mathrm{Ru}(1)-\mathrm{N}(1)$ & $101.66(7)$ & $\mathrm{C}(1)-\mathrm{C}(5)-\mathrm{Ru}(1)$ & $78.07(11)$ \\
\hline $\mathrm{C}(32)-\mathrm{Ru}(1)-\mathrm{N}(1)$ & $94.15(7)$ & $C(26)-C(5)-R u(1)$ & $128.52(14)$ \\
\hline $\mathrm{C}(4)-\mathrm{Ru}(1)-\mathrm{N}(1)$ & 131.61(6) & $\mathrm{C}(11)-\mathrm{C}(6)-\mathrm{C}(7)$ & $117.83(19)$ \\
\hline $\mathrm{C}(3)-\mathrm{Ru}(1)-\mathrm{N}(1)$ & $135.00(7)$ & $\mathrm{C}(11)-\mathrm{C}(6)-\mathrm{C}(2)$ & $121.98(18)$ \\
\hline$C(33)-R u(1)-C(2)$ & $98.70(8)$ & $C(7)-C(6)-C(2)$ & $120.17(18)$ \\
\hline$C(32)-R u(1)-C(2)$ & $163.20(8)$ & $C(8)-C(7)-C(6)$ & $120.2(2)$ \\
\hline $\mathrm{C}(4)-\mathrm{Ru}(1)-\mathrm{C}(2)$ & $63.43(7)$ & $\mathrm{C}(8)-\mathrm{C}(7)-\mathrm{H}(7)$ & 119.9 \\
\hline$C(3)-R u(1)-C(2)$ & $37.91(7)$ & $\mathrm{C}(6)-\mathrm{C}(7)-\mathrm{H}(7)$ & 119.9 \\
\hline $\mathrm{N}(1)-\mathrm{Ru}(1)-\mathrm{C}(2)$ & $97.79(6)$ & $C(9)-C(8)-C(7)$ & $121.4(2)$ \\
\hline $\mathrm{C}(33)-\mathrm{Ru}(1)-\mathrm{C}(5)$ & $157.64(8)$ & $\mathrm{C}(9)-\mathrm{C}(8)-\mathrm{H}(8)$ & 119.3 \\
\hline $\mathrm{C}(32)-\mathrm{Ru}(1)-\mathrm{C}(5)$ & $104.57(8)$ & $\mathrm{C}(7)-\mathrm{C}(8)-\mathrm{H}(8)$ & 119.3 \\
\hline$C(4)-R u(1)-C(5)$ & $37.79(7)$ & $\mathrm{C}(8)-\mathrm{C}(9)-\mathrm{C}(10)$ & $118.8(2)$ \\
\hline$C(3)-R u(1)-C(5)$ & $63.05(7)$ & $\mathrm{C}(8)-\mathrm{C}(9)-\mathrm{H}(9)$ & 120.6 \\
\hline $\mathrm{N}(1)-\mathrm{Ru}(1)-\mathrm{C}(5)$ & $93.86(6)$ & $\mathrm{C}(10)-\mathrm{C}(9)-\mathrm{H}(9)$ & 120.6 \\
\hline$C(2)-R u(1)-C(5)$ & $62.96(7)$ & $\mathrm{C}(11)-\mathrm{C}(10)-\mathrm{C}(9)$ & $120.5(2)$ \\
\hline$C(33)-R u(1)-C(1)$ & $132.34(8)$ & $\mathrm{C}(11)-\mathrm{C}(10)-\mathrm{H}(10)$ & 119.7 \\
\hline$C(32)-R u(1)-C(1)$ & $137.24(8)$ & $\mathrm{C}(9)-\mathrm{C}(10)-\mathrm{H}(10)$ & 119.7 \\
\hline $\mathrm{C}(4)-\mathrm{Ru}(1)-\mathrm{C}(1)$ & $61.13(7)$ & $\mathrm{C}(10)-\mathrm{C}(11)-\mathrm{C}(6)$ & $121.20(19)$ \\
\hline$C(3)-R u(1)-C(1)$ & $61.07(7)$ & $\mathrm{C}(10)-\mathrm{C}(11)-\mathrm{H}(11)$ & 119.4 \\
\hline $\mathrm{N}(1)-\mathrm{Ru}(1)-\mathrm{C}(1)$ & $77.36(6)$ & $\mathrm{C}(6)-\mathrm{C}(11)-\mathrm{H}(11)$ & 119.4 \\
\hline$C(2)-R u(1)-C(1)$ & $36.61(7)$ & $\mathrm{C}(18)-\mathrm{C}(12)-\mathrm{C}(13)$ & $118.06(18)$ \\
\hline $\mathrm{C}(5)-\mathrm{Ru}(1)-\mathrm{C}(1)$ & $36.38(7)$ & $\mathrm{C}(18)-\mathrm{C}(12)-\mathrm{C}(3)$ & $123.08(18)$ \\
\hline $\mathrm{C}(41)-\mathrm{N}(1)-\mathrm{C}(34)$ & $112.32(14)$ & $\mathrm{C}(13)-\mathrm{C}(12)-\mathrm{C}(3)$ & $118.82(18)$ \\
\hline $\mathrm{C}(41)-\mathrm{N}(1)-\mathrm{Ru}(1)$ & $122.93(11)$ & $\mathrm{C}(14)-\mathrm{C}(13)-\mathrm{C}(12)$ & $120.8(2)$ \\
\hline $\mathrm{C}(34)-\mathrm{N}(1)-\mathrm{Ru}(1)$ & $110.11(11)$ & $\mathrm{C}(14)-\mathrm{C}(13)-\mathrm{H}(13)$ & 119.6 \\
\hline $\mathrm{C}(41)-\mathrm{N}(1)-\mathrm{H}(1)$ & 102.9 & $\mathrm{C}(12)-\mathrm{C}(13)-\mathrm{H}(13)$ & 119.6 \\
\hline $\mathrm{C}(34)-\mathrm{N}(1)-\mathrm{H}(1)$ & 102.9 & $\mathrm{C}(13)-\mathrm{C}(14)-\mathrm{C}(15)$ & 121.1(2) \\
\hline $\mathrm{Ru}(1)-\mathrm{N}(1)-\mathrm{H}(1)$ & 102.9 & $\mathrm{C}(13)-\mathrm{C}(14)-\mathrm{H}(14)$ & 119.5 \\
\hline $\mathrm{O}(1)-\mathrm{C}(1)-\mathrm{C}(5)$ & 126.11(18) & $\mathrm{C}(15)-\mathrm{C}(14)-\mathrm{H}(14)$ & 119.5 \\
\hline $\mathrm{O}(1)-\mathrm{C}(1)-\mathrm{C}(2)$ & $126.78(19)$ & $C(17)-C(15)-C(14)$ & $118.19(19)$ \\
\hline$C(5)-C(1)-C(2)$ & $105.98(16)$ & $\mathrm{C}(17)-\mathrm{C}(15)-\mathrm{C}(16)$ & $121.4(2)$ \\
\hline $\mathrm{O}(1)-\mathrm{C}(1)-\mathrm{Ru}(1)$ & $124.39(13)$ & $C(14)-C(15)-C(16)$ & $120.4(2)$ \\
\hline $\mathrm{C}(5)-\mathrm{C}(1)-\mathrm{Ru}(1)$ & $65.55(10)$ & $\mathrm{C}(15)-\mathrm{C}(16)-\mathrm{H}(16 \mathrm{~A})$ & 109.5 \\
\hline $\mathrm{C}(2)-\mathrm{C}(1)-\mathrm{Ru}(1)$ & $65.33(10)$ & $\mathrm{C}(15)-\mathrm{C}(16)-\mathrm{H}(16 \mathrm{~B})$ & 109.5 \\
\hline$C(3)-C(2)-C(6)$ & $127.06(17)$ & H(16A)-C(16)-H(16B) & 109.5 \\
\hline$C(3)-C(2)-C(1)$ & $107.59(17)$ & $\mathrm{C}(15)-\mathrm{C}(16)-\mathrm{H}(16 \mathrm{C})$ & 109.5 \\
\hline$C(6)-C(2)-C(1)$ & $124.86(17)$ & $\mathrm{H}(16 \mathrm{~A})-\mathrm{C}(16)-\mathrm{H}(16 \mathrm{C})$ & 109.5 \\
\hline$C(3)-C(2)-R u(1)$ & $69.63(11)$ & H(16B)-C(16)-H(16C) & 109.5 \\
\hline$C(6)-C(2)-R u(1)$ & $124.29(13)$ & $\mathrm{C}(15)-\mathrm{C}(17)-\mathrm{C}(18)$ & $121.2(2)$ \\
\hline$C(1)-C(2)-R u(1)$ & $78.06(11)$ & $\mathrm{C}(15)-\mathrm{C}(17)-\mathrm{H}(17)$ & 119.4 \\
\hline $\mathrm{C}(4)-\mathrm{C}(3)-\mathrm{C}(2)$ & $108.40(16)$ & $\mathrm{C}(18)-\mathrm{C}(17)-\mathrm{H}(17)$ & 119.4 \\
\hline$C(4)-C(3)-C(12)$ & $124.75(17)$ & $\mathrm{C}(12)-\mathrm{C}(18)-\mathrm{C}(17)$ & $120.7(2)$ \\
\hline$C(2)-C(3)-C(12)$ & $125.83(18)$ & $\mathrm{C}(12)-\mathrm{C}(18)-\mathrm{H}(18)$ & 119.7 \\
\hline$C(4)-C(3)-R u(1)$ & 70.41(11) & $\mathrm{C}(17)-\mathrm{C}(18)-\mathrm{H}(18)$ & 119.7 \\
\hline$C(2)-C(3)-R u(1)$ & $72.46(11)$ & $C(25)-C(19)-C(20)$ & $118.2(2)$ \\
\hline$C(12)-C(3)-R u(1)$ & $131.95(13)$ & $C(25)-C(19)-C(4)$ & $118.15(19)$ \\
\hline$C(3)-C(4)-C(5)$ & $108.69(17)$ & $\mathrm{C}(20)-\mathrm{C}(19)-\mathrm{C}(4)$ & $123.69(19)$ \\
\hline$C(3)-C(4)-C(19)$ & 124.91(17) & $\mathrm{C}(21)-\mathrm{C}(20)-\mathrm{C}(19)$ & $120.7(2)$ \\
\hline$C(5)-C(4)-C(19)$ & $125.12(18)$ & $\mathrm{C}(21)-\mathrm{C}(20)-\mathrm{H}(20)$ & 119.6 \\
\hline$C(3)-C(4)-R u(1)$ & $71.67(11)$ & $\mathrm{C}(19)-\mathrm{C}(20)-\mathrm{H}(20)$ & 119.6 \\
\hline$C(5)-C(4)-R u(1)$ & $73.30(11)$ & $\mathrm{C}(22)-\mathrm{C}(21)-\mathrm{C}(20)$ & $121.5(2)$ \\
\hline$C(19)-C(4)-R u(1)$ & $131.22(14)$ & $\mathrm{C}(22)-\mathrm{C}(21)-\mathrm{H}(21)$ & 119.3 \\
\hline
\end{tabular}




\begin{tabular}{|c|c|}
\hline $\mathrm{C}(20)-\mathrm{C}(21)-\mathrm{H}(21)$ & 119.3 \\
\hline $\mathrm{C}(21)-\mathrm{C}(22)-\mathrm{C}(24)$ & $117.6(2)$ \\
\hline$C(21)-C(22)-C(23)$ & $120.8(2)$ \\
\hline$C(24)-C(22)-C(23)$ & $121.6(2)$ \\
\hline $\mathrm{C}(22)-\mathrm{C}(23)-\mathrm{H}(23 \mathrm{~A})$ & 109.5 \\
\hline $\mathrm{C}(22)-\mathrm{C}(23)-\mathrm{H}(23 \mathrm{~B})$ & 109.5 \\
\hline $\mathrm{H}(23 \mathrm{~A})-\mathrm{C}(23)-\mathrm{H}(23 \mathrm{~B})$ & 109.5 \\
\hline $\mathrm{C}(22)-\mathrm{C}(23)-\mathrm{H}(23 \mathrm{C})$ & 109.5 \\
\hline $\mathrm{H}(23 \mathrm{~A})-\mathrm{C}(23)-\mathrm{H}(23 \mathrm{C})$ & 109.5 \\
\hline $\mathrm{H}(23 \mathrm{~B})-\mathrm{C}(23)-\mathrm{H}(23 \mathrm{C})$ & 109.5 \\
\hline$C(25)-C(24)-C(22)$ & $121.4(2)$ \\
\hline $\mathrm{C}(25)-\mathrm{C}(24)-\mathrm{H}(24)$ & 119.3 \\
\hline $\mathrm{C}(22)-\mathrm{C}(24)-\mathrm{H}(24)$ & 119.3 \\
\hline$C(19)-C(25)-C(24)$ & $120.6(2)$ \\
\hline $\mathrm{C}(19)-\mathrm{C}(25)-\mathrm{H}(25)$ & 119.7 \\
\hline $\mathrm{C}(24)-\mathrm{C}(25)-\mathrm{H}(25)$ & 119.7 \\
\hline $\mathrm{C}(31)-\mathrm{C}(26)-\mathrm{C}(27)$ & $118.2(2)$ \\
\hline$C(31)-C(26)-C(5)$ & $119.6(2)$ \\
\hline$C(27)-C(26)-C(5)$ & $122.27(19)$ \\
\hline $\mathrm{C}(28)-\mathrm{C}(27)-\mathrm{C}(26)$ & $120.8(2)$ \\
\hline $\mathrm{C}(28)-\mathrm{C}(27)-\mathrm{H}(27)$ & 119.6 \\
\hline $\mathrm{C}(26)-\mathrm{C}(27)-\mathrm{H}(27)$ & 119.6 \\
\hline $\mathrm{C}(29)-\mathrm{C}(28)-\mathrm{C}(27)$ & $120.2(3)$ \\
\hline $\mathrm{C}(29)-\mathrm{C}(28)-\mathrm{H}(28)$ & 119.9 \\
\hline $\mathrm{C}(27)-\mathrm{C}(28)-\mathrm{H}(28)$ & 119.9 \\
\hline $\mathrm{C}(30)-\mathrm{C}(29)-\mathrm{C}(28)$ & $119.7(2)$ \\
\hline $\mathrm{C}(30)-\mathrm{C}(29)-\mathrm{H}(29)$ & 120.2 \\
\hline $\mathrm{C}(28)-\mathrm{C}(29)-\mathrm{H}(29)$ & 120.2 \\
\hline $\mathrm{C}(29)-\mathrm{C}(30)-\mathrm{C}(31)$ & $120.6(2)$ \\
\hline $\mathrm{C}(29)-\mathrm{C}(30)-\mathrm{H}(30)$ & 119.7 \\
\hline $\mathrm{C}(31)-\mathrm{C}(30)-\mathrm{H}(30)$ & 119.7 \\
\hline $\mathrm{C}(30)-\mathrm{C}(31)-\mathrm{C}(26)$ & $120.6(2)$ \\
\hline $\mathrm{C}(30)-\mathrm{C}(31)-\mathrm{H}(31)$ & 119.7 \\
\hline $\mathrm{C}(26)-\mathrm{C}(31)-\mathrm{H}(31)$ & 119.7 \\
\hline $\mathrm{O}(3)-\mathrm{C}(32)-\mathrm{Ru}(1)$ & $178.08(18)$ \\
\hline $\mathrm{O}(2)-\mathrm{C}(33)-\mathrm{Ru}(1)$ & $173.96(17)$ \\
\hline $\mathrm{N}(1)-\mathrm{C}(34)-\mathrm{C}(35)$ & $114.30(16)$ \\
\hline $\mathrm{N}(1)-\mathrm{C}(34)-\mathrm{H}(34 \mathrm{~A})$ & 108.7 \\
\hline $\mathrm{C}(35)-\mathrm{C}(34)-\mathrm{H}(34 \mathrm{~A})$ & 108.7 \\
\hline $\mathrm{N}(1)-\mathrm{C}(34)-\mathrm{H}(34 \mathrm{~B})$ & 108.7 \\
\hline $\mathrm{C}(35)-\mathrm{C}(34)-\mathrm{H}(34 \mathrm{~B})$ & 108.7 \\
\hline $\mathrm{H}(34 \mathrm{~A})-\mathrm{C}(34)-\mathrm{H}(34 \mathrm{~B})$ & 107.6 \\
\hline$C(40)-C(35)-C(36)$ & $118.97(18)$ \\
\hline $\mathrm{C}(40)-\mathrm{C}(35)-\mathrm{C}(34)$ & $121.70(18)$ \\
\hline $\mathrm{C}(36)-\mathrm{C}(35)-\mathrm{C}(34)$ & $119.31(18)$ \\
\hline $\mathrm{C}(37)-\mathrm{C}(36)-\mathrm{C}(35)$ & $120.19(19)$ \\
\hline $\mathrm{C}(37)-\mathrm{C}(36)-\mathrm{H}(36)$ & 119.9 \\
\hline $\mathrm{C}(35)-\mathrm{C}(36)-\mathrm{H}(36)$ & 119.9 \\
\hline $\mathrm{C}(38)-\mathrm{C}(37)-\mathrm{C}(36)$ & $120.6(2)$ \\
\hline $\mathrm{C}(38)-\mathrm{C}(37)-\mathrm{H}(37)$ & 119.7 \\
\hline $\mathrm{C}(36)-\mathrm{C}(37)-\mathrm{H}(37)$ & 119.7 \\
\hline $\mathrm{C}(37)-\mathrm{C}(38)-\mathrm{C}(39)$ & $119.60(19)$ \\
\hline $\mathrm{C}(37)-\mathrm{C}(38)-\mathrm{H}(38)$ & 120.2 \\
\hline $\mathrm{C}(39)-\mathrm{C}(38)-\mathrm{H}(38)$ & 120.2 \\
\hline $\mathrm{C}(38)-\mathrm{C}(39)-\mathrm{C}(40)$ & $119.9(2)$ \\
\hline $\mathrm{C}(38)-\mathrm{C}(39)-\mathrm{H}(39)$ & 120.1 \\
\hline
\end{tabular}

$\mathrm{C}(40)-\mathrm{C}(39)-\mathrm{H}(39) \quad 120.1$

$\mathrm{C}(35)-\mathrm{C}(40)-\mathrm{C}(39) \quad 120.7(2)$

$\mathrm{C}(35)-\mathrm{C}(40)-\mathrm{H}(40) \quad 119.7$

$\mathrm{C}(39)-\mathrm{C}(40)-\mathrm{H}(40) \quad 119.7$

$\mathrm{N}(1)-\mathrm{C}(41)-\mathrm{C}(42) \quad 110.59(15)$

$\mathrm{N}(1)-\mathrm{C}(41)-\mathrm{C}(43) \quad 113.11(13)$

$\mathrm{C}(42)-\mathrm{C}(41)-\mathrm{C}(43) \quad 110.77(14)$

$\mathrm{N}(1)-\mathrm{C}(41)-\mathrm{H}(41) \quad 107.4$

$\mathrm{C}(42)-\mathrm{C}(41)-\mathrm{H}(41) \quad 107.4$

$\mathrm{C}(43)-\mathrm{C}(41)-\mathrm{H}(41) \quad 107.4$

$\mathrm{C}(41)-\mathrm{C}(42)-\mathrm{C}(43) \# 1 \quad 110.72(13)$

$\mathrm{C}(41)-\mathrm{C}(42)-\mathrm{H}(42 \mathrm{~A}) \quad 109.5$

$\mathrm{C}(43) \# 1-\mathrm{C}(42)-\mathrm{H}(42 \mathrm{~A}) \quad 109.5$

$\mathrm{C}(41)-\mathrm{C}(42)-\mathrm{H}(42 \mathrm{~B}) \quad 109.5$

C(43)\#1-C(42)-H(42B) $\quad 109.5$

$\mathrm{H}(42 \mathrm{~A})-\mathrm{C}(42)-\mathrm{H}(42 \mathrm{~B})$

$\mathrm{C}(41)-\mathrm{C}(43)-\mathrm{C}(42) \# 1 \quad 110.14(10)$

$\mathrm{C}(41)-\mathrm{C}(43)-\mathrm{H}(43 \mathrm{~A}) \quad 109.6$

$\mathrm{C}(42) \# 1-\mathrm{C}(43)-\mathrm{H}(43 \mathrm{~A}) \quad 109.6$

$\mathrm{C}(41)-\mathrm{C}(43)-\mathrm{H}(43 \mathrm{~B}) \quad 109.6$

$\mathrm{C}(42) \# 1-\mathrm{C}(43)-\mathrm{H}(43 \mathrm{~B}) \quad 109.6$

$\mathrm{H}(43 \mathrm{~A})-\mathrm{C}(43)-\mathrm{H}(43 \mathrm{~B}) \quad 108.1$

$\mathrm{Cl}(1)-\mathrm{C}(44)-\mathrm{Cl}(2) \quad 111.7$

$\mathrm{Cl}(1)-\mathrm{C}(44)-\mathrm{H}(44 \mathrm{~A}) \quad 109.3$

$\mathrm{Cl}(2)-\mathrm{C}(44)-\mathrm{H}(44 \mathrm{~A}) \quad 109.3$

$\mathrm{Cl}(1)-\mathrm{C}(44)-\mathrm{H}(44 \mathrm{~B}) \quad 109.3$

$\mathrm{Cl}(2)-\mathrm{C}(44)-\mathrm{H}(44 \mathrm{~B}) \quad 109.3$

H(44A)-C(44)-H(44B) $\quad 107.9$

Symmetry transformations used to generate equivalent atoms: $\quad \# 1-\mathrm{x},-\mathrm{y}+2,-\mathrm{z}^{+}$ 
Table 4. Anisotropic displacement parameters $\left(\AA^{2} \times 10^{3}\right)$ for $\mathbf{2 3} \mathbf{\bullet}\left(\mathbf{C H}_{\mathbf{2}} \mathbf{C l}_{\mathbf{2}}\right)_{\mathbf{2}}$. The anisotropic displacement factor exponent takes the form: $-2 \pi^{2}\left[h^{2} a^{* 2} U^{11}+\ldots+2 h k a^{*} b^{*} U^{12}\right]$

\begin{tabular}{|c|c|c|c|c|c|c|}
\hline & $\mathrm{U}^{11}$ & $\mathrm{U}^{22}$ & $\mathrm{U}^{33}$ & $\mathrm{U}^{23}$ & $\mathrm{U}^{13}$ & $\mathrm{U}^{12}$ \\
\hline$\overline{R u(1)}$ & 16(1) & 11(1) & $15(1)$ & $-5(1)$ & $2(1)$ & $-3(1)$ \\
\hline $\mathrm{O}(1)$ & 24(1) & $14(1)$ & 23(1) & $-7(1)$ & $5(1)$ & $-6(1)$ \\
\hline $\mathrm{O}(2)$ & $30(1)$ & $17(1)$ & $26(1)$ & $0(1)$ & $4(1)$ & $-3(1)$ \\
\hline $\mathrm{O}(3)$ & $22(1)$ & $33(1)$ & $33(1)$ & $-13(1)$ & $1(1)$ & $-8(1)$ \\
\hline $\mathrm{N}(1)$ & $16(1)$ & 12(1) & $16(1)$ & $-4(1)$ & $2(1)$ & $-2(1)$ \\
\hline $\mathrm{C}(1)$ & $20(1)$ & $15(1)$ & 18(1) & $-3(1)$ & $8(1)$ & $-6(1)$ \\
\hline $\mathrm{C}(2)$ & 19(1) & $15(1)$ & $17(1)$ & $-5(1)$ & $5(1)$ & $-4(1)$ \\
\hline C(3) & $18(1)$ & $15(1)$ & 18(1) & $-5(1)$ & $6(1)$ & $-6(1)$ \\
\hline$C(4)$ & 21(1) & $15(1)$ & $18(1)$ & $-5(1)$ & $5(1)$ & $-3(1)$ \\
\hline$C(5)$ & 20(1) & $16(1)$ & $16(1)$ & $-5(1)$ & $5(1)$ & $-4(1)$ \\
\hline$C(6)$ & $16(1)$ & $20(1)$ & $20(1)$ & $-9(1)$ & $6(1)$ & $-4(1)$ \\
\hline$C(7)$ & $18(1)$ & $20(1)$ & $26(1)$ & $-7(1)$ & $6(1)$ & $-4(1)$ \\
\hline $\mathrm{C}(8)$ & $18(1)$ & $27(1)$ & $30(1)$ & $-12(1)$ & $3(1)$ & $-7(1)$ \\
\hline$C(9)$ & $16(1)$ & $34(1)$ & $25(1)$ & $-10(1)$ & $0(1)$ & $-3(1)$ \\
\hline $\mathrm{C}(10)$ & $18(1)$ & $25(1)$ & $24(1)$ & $-6(1)$ & $3(1)$ & $0(1)$ \\
\hline $\mathrm{C}(11)$ & $20(1)$ & 19(1) & 23(1) & $-8(1)$ & $4(1)$ & $-4(1)$ \\
\hline$C(12)$ & $22(1)$ & $15(1)$ & $16(1)$ & $-6(1)$ & $4(1)$ & $-1(1)$ \\
\hline$C(13)$ & $24(1)$ & $20(1)$ & $27(1)$ & $-8(1)$ & $6(1)$ & $-4(1)$ \\
\hline$C(14)$ & $20(1)$ & $28(1)$ & $36(1)$ & $-11(1)$ & $6(1)$ & $0(1)$ \\
\hline $\mathrm{C}(15)$ & $33(1)$ & $20(1)$ & $24(1)$ & $-7(1)$ & $5(1)$ & $3(1)$ \\
\hline$C(16)$ & $41(2)$ & $22(1)$ & $52(2)$ & $-14(1)$ & $5(1)$ & $7(1)$ \\
\hline$C(17)$ & $32(1)$ & $16(1)$ & $22(1)$ & $-7(1)$ & $3(1)$ & $-4(1)$ \\
\hline $\mathrm{C}(18)$ & $22(1)$ & 19(1) & $20(1)$ & $-6(1)$ & $3(1)$ & $-4(1)$ \\
\hline$C(19)$ & $26(1)$ & $16(1)$ & $16(1)$ & $-6(1)$ & $-1(1)$ & $2(1)$ \\
\hline$C(20)$ & $34(1)$ & 21(1) & 20(1) & $-8(1)$ & $3(1)$ & $-5(1)$ \\
\hline $\mathrm{C}(21)$ & $33(1)$ & 21(1) & $28(1)$ & $-11(1)$ & $-3(1)$ & $-4(1)$ \\
\hline $\mathrm{C}(22)$ & $40(1)$ & 31(1) & 23(1) & $-10(1)$ & $-6(1)$ & $-1(1)$ \\
\hline$C(23)$ & 61(2) & $57(2)$ & $26(1)$ & $-19(1)$ & $-5(1)$ & $-13(2)$ \\
\hline$C(24)$ & $49(2)$ & $51(2)$ & $17(1)$ & $-8(1)$ & $5(1)$ & $-13(1)$ \\
\hline$C(25)$ & $35(1)$ & $35(1)$ & 23(1) & $-6(1)$ & $4(1)$ & $-12(1)$ \\
\hline$C(26)$ & $29(1)$ & 18(1) & 17(1) & $-5(1)$ & $5(1)$ & $-1(1)$ \\
\hline$C(27)$ & 31(1) & $24(1)$ & 24(1) & $-2(1)$ & $2(1)$ & 1(1) \\
\hline$C(28)$ & $32(1)$ & 41(1) & $33(1)$ & $-3(1)$ & $-2(1)$ & $5(1)$ \\
\hline $\mathrm{C}(29)$ & $55(2)$ & $29(1)$ & $34(1)$ & $4(1)$ & $-3(1)$ & $13(1)$ \\
\hline $\mathrm{C}(30)$ & $64(2)$ & 18(1) & $32(1)$ & $-1(1)$ & $1(1)$ & $0(1)$ \\
\hline$C(31)$ & 41(1) & 19(1) & 20(1) & $-3(1)$ & $2(1)$ & $-3(1)$ \\
\hline$C(32)$ & $25(1)$ & 16(1) & 17(1) & $-7(1)$ & $3(1)$ & $-4(1)$ \\
\hline $\mathrm{C}(33)$ & $17(1)$ & $17(1)$ & $20(1)$ & $-7(1)$ & $2(1)$ & $-3(1)$ \\
\hline$C(34)$ & $17(1)$ & 16(1) & 18(1) & $-6(1)$ & $-1(1)$ & $0(1)$ \\
\hline$C(35)$ & $18(1)$ & $15(1)$ & $17(1)$ & $-6(1)$ & $-2(1)$ & $0(1)$ \\
\hline$C(36)$ & $24(1)$ & 19(1) & $22(1)$ & $-4(1)$ & $5(1)$ & $-1(1)$ \\
\hline $\mathrm{C}(37)$ & $24(1)$ & $18(1)$ & $32(1)$ & $-5(1)$ & $2(1)$ & $-4(1)$ \\
\hline $\mathrm{C}(38)$ & $25(1)$ & $16(1)$ & $37(1)$ & $-12(1)$ & $0(1)$ & 1(1) \\
\hline$C(39)$ & 21(1) & $25(1)$ & $32(1)$ & $-14(1)$ & $7(1)$ & $-2(1)$ \\
\hline $\mathrm{C}(40)$ & 19(1) & 19(1) & $26(1)$ & $-7(1)$ & $3(1)$ & $-4(1)$ \\
\hline $\mathrm{C}(41)$ & $16(1)$ & $14(1)$ & 14(1) & $-5(1)$ & $0(1)$ & $-2(1)$ \\
\hline$C(42)$ & $15(1)$ & $15(1)$ & $17(1)$ & $-5(1)$ & $2(1)$ & $-3(1)$ \\
\hline C(43) & $14(1)$ & $16(1)$ & $17(1)$ & $-6(1)$ & $2(1)$ & $-4(1)$ \\
\hline $\mathrm{Cl}(1)$ & $36(1)$ & $24(1)$ & $27(1)$ & $-7(1)$ & $7(1)$ & $-11(1)$ \\
\hline $\mathrm{Cl}(2)$ & $27(1)$ & 61(1) & 31(1) & $-7(1)$ & $-1(1)$ & $-8(1)$ \\
\hline $\mathrm{C}(44)$ & $26(1)$ & $24(1)$ & $24(1)$ & $-7(1)$ & $2(1)$ & $-6(1)$ \\
\hline
\end{tabular}


Table 5. Hydrogen coordinates ( $\left.\times 10^{4}\right)$ and isotropic displacement parameters $\left(\AA^{2} \times 10^{3}\right)$ for $\mathbf{2 3} \bullet\left(\mathrm{CH}_{2} \mathrm{Cl}_{2}\right)_{2}$.

\begin{tabular}{|c|c|c|c|c|}
\hline & $\mathrm{x}$ & $y$ & $\mathrm{z}$ & $\mathrm{U}(\mathrm{eq})$ \\
\hline $\mathrm{H}(1)$ & 356 & 11511 & 8547 & 17 \\
\hline $\mathrm{H}(7)$ & 4074 & 11142 & 8354 & 25 \\
\hline $\mathrm{H}(8)$ & 5590 & 10739 & 9186 & 29 \\
\hline $\mathrm{H}(9)$ & 6037 & 8671 & 9791 & 29 \\
\hline $\mathrm{H}(10)$ & 4898 & 6991 & 9571 & 27 \\
\hline $\mathrm{H}(11)$ & 3343 & 7381 & 8759 & 24 \\
\hline $\mathrm{H}(13)$ & 4771 & 7912 & 7514 & 27 \\
\hline $\mathrm{H}(14)$ & 5992 & 5878 & 7449 & 34 \\
\hline $\mathrm{H}(16 \mathrm{~A})$ & 5514 & 3194 & 7880 & 58 \\
\hline $\mathrm{H}(16 \mathrm{~B})$ & 5935 & 3656 & 7164 & 58 \\
\hline $\mathrm{H}(16 \mathrm{C})$ & 4577 & 2994 & 7326 & 58 \\
\hline $\mathrm{H}(17)$ & 2471 & 4338 & 7498 & 28 \\
\hline $\mathrm{H}(18)$ & 1234 & 6367 & 7574 & 24 \\
\hline $\mathrm{H}(20)$ & -862 & 7832 & 7058 & 30 \\
\hline $\mathrm{H}(21)$ & -1571 & 7113 & 6181 & 32 \\
\hline $\mathrm{H}(23 \mathrm{~A})$ & -347 & 6509 & 5025 & 69 \\
\hline $\mathrm{H}(23 \mathrm{~B})$ & -336 & 7923 & 4612 & 69 \\
\hline $\mathrm{H}(23 \mathrm{C})$ & -1694 & 7560 & 4983 & 69 \\
\hline $\mathrm{H}(24)$ & 1236 & 8935 & 5120 & 46 \\
\hline $\mathrm{H}(25)$ & 1935 & 9683 & 5991 & 37 \\
\hline $\mathrm{H}(27)$ & -1614 & 10776 & 6735 & 32 \\
\hline $\mathrm{H}(28)$ & -2964 & 12501 & 6125 & 44 \\
\hline $\mathrm{H}(29)$ & -2252 & 14555 & 5901 & 50 \\
\hline $\mathrm{H}(30)$ & -142 & 14859 & 6251 & 47 \\
\hline $\mathrm{H}(31)$ & 1232 & 13145 & 6852 & 32 \\
\hline $\mathrm{H}(34 \mathrm{~A})$ & -2387 & 11337 & 8580 & 20 \\
\hline $\mathrm{H}(34 \mathrm{~B})$ & -1593 & 11870 & 7955 & 20 \\
\hline $\mathrm{H}(36)$ & -719 & 13967 & 7850 & 26 \\
\hline $\mathrm{H}(37)$ & -1100 & 16083 & 8079 & 30 \\
\hline $\mathrm{H}(38)$ & -2400 & 16540 & 8953 & 31 \\
\hline H(39) & -3324 & 14861 & 9611 & 30 \\
\hline $\mathrm{H}(40)$ & -3007 & 12752 & 9366 & 25 \\
\hline $\mathrm{H}(41)$ & -488 & 11638 & 9500 & 17 \\
\hline $\mathrm{H}(42 \mathrm{~A})$ & 1840 & 10814 & 9394 & 19 \\
\hline $\mathrm{H}(42 \mathrm{~B})$ & 1468 & 9359 & 9473 & 19 \\
\hline $\mathrm{H}(43 \mathrm{~A})$ & -1072 & 9021 & 9569 & 18 \\
\hline $\mathrm{H}(43 \mathrm{~B})$ & -2208 & 10277 & 9550 & 18 \\
\hline $\mathrm{H}(44 \mathrm{~A})$ & 7937 & 6791 & 1277 & 29 \\
\hline $\mathrm{H}(44 \mathrm{~B})$ & 8288 & 5251 & 1292 & 29 \\
\hline
\end{tabular}


Table 6. Torsion angles $\left[^{\circ}\right]$ for $\mathbf{2 3 \bullet}\left(\mathbf{C H}_{\mathbf{2}} \mathbf{C l}_{2}\right)_{2}$.

\begin{tabular}{|c|c|}
\hline $\mathrm{C}(33)-\mathrm{Ru}(1)-\mathrm{N}(1)-\mathrm{C}(41)$ & $9.63(15)$ \\
\hline $\mathrm{C}(32)-\mathrm{Ru}(1)-\mathrm{N}(1)-\mathrm{C}(41)$ & $100.88(15)$ \\
\hline $\mathrm{C}(4)-\mathrm{Ru}(1)-\mathrm{N}(1)-\mathrm{C}(41)$ & $-152.13(13)$ \\
\hline $\mathrm{C}(3)-\mathrm{Ru}(1)-\mathrm{N}(1)-\mathrm{C}(41)$ & $-99.28(15)$ \\
\hline $\mathrm{C}(2)-\mathrm{Ru}(1)-\mathrm{N}(1)-\mathrm{C}(41)$ & $-90.97(14)$ \\
\hline $\mathrm{C}(5)-\mathrm{Ru}(1)-\mathrm{N}(1)-\mathrm{C}(41)$ & $-154.19(14)$ \\
\hline $\mathrm{C}(1)-\mathrm{Ru}(1)-\mathrm{N}(1)-\mathrm{C}(41)$ & $-121.55(14)$ \\
\hline $\mathrm{C}(33)-\mathrm{Ru}(1)-\mathrm{N}(1)-\mathrm{C}(34)$ & $-126.34(13)$ \\
\hline $\mathrm{C}(32)-\mathrm{Ru}(1)-\mathrm{N}(1)-\mathrm{C}(34)$ & $-35.09(13)$ \\
\hline $\mathrm{C}(4)-\mathrm{Ru}(1)-\mathrm{N}(1)-\mathrm{C}(34)$ & $71.90(15)$ \\
\hline $\mathrm{C}(3)-\mathrm{Ru}(1)-\mathrm{N}(1)-\mathrm{C}(34)$ & $124.74(13)$ \\
\hline $\mathrm{C}(2)-\mathrm{Ru}(1)-\mathrm{N}(1)-\mathrm{C}(34)$ & $133.06(12)$ \\
\hline $\mathrm{C}(5)-\mathrm{Ru}(1)-\mathrm{N}(1)-\mathrm{C}(34)$ & $69.84(13)$ \\
\hline $\mathrm{C}(1)-\mathrm{Ru}(1)-\mathrm{N}(1)-\mathrm{C}(34)$ & $102.48(13)$ \\
\hline $\mathrm{C}(33)-\mathrm{Ru}(1)-\mathrm{C}(1)-\mathrm{O}(1)$ & $-90.98(19)$ \\
\hline $\mathrm{C}(32)-\mathrm{Ru}(1)-\mathrm{C}(1)-\mathrm{O}(1)$ & $85.7(2)$ \\
\hline $\mathrm{C}(4)-\mathrm{Ru}(1)-\mathrm{C}(1)-\mathrm{O}(1)$ & $157.6(2)$ \\
\hline $\mathrm{C}(3)-\mathrm{Ru}(1)-\mathrm{C}(1)-\mathrm{O}(1)$ & $-158.8(2)$ \\
\hline $\mathrm{N}(1)-\mathrm{Ru}(1)-\mathrm{C}(1)-\mathrm{O}(1)$ & $3.37(17)$ \\
\hline $\mathrm{C}(2)-\mathrm{Ru}(1)-\mathrm{C}(1)-\mathrm{O}(1)$ & $-118.9(2)$ \\
\hline $\mathrm{C}(5)-\mathrm{Ru}(1)-\mathrm{C}(1)-\mathrm{O}(1)$ & $118.3(2)$ \\
\hline $\mathrm{C}(33)-\mathrm{Ru}(1)-\mathrm{C}(1)-\mathrm{C}(5)$ & $150.77(12)$ \\
\hline $\mathrm{C}(32)-\mathrm{Ru}(1)-\mathrm{C}(1)-\mathrm{C}(5)$ & $-32.54(16)$ \\
\hline $\mathrm{C}(4)-\mathrm{Ru}(1)-\mathrm{C}(1)-\mathrm{C}(5)$ & $39.37(11)$ \\
\hline$C(3)-R u(1)-C(1)-C(5)$ & $82.94(12)$ \\
\hline $\mathrm{N}(1)-\mathrm{Ru}(1)-\mathrm{C}(1)-\mathrm{C}(5)$ & $-114.88(11)$ \\
\hline $\mathrm{C}(2)-\mathrm{Ru}(1)-\mathrm{C}(1)-\mathrm{C}(5)$ & $122.80(16)$ \\
\hline $\mathrm{C}(33)-\mathrm{Ru}(1)-\mathrm{C}(1)-\mathrm{C}(2)$ & $27.97(15)$ \\
\hline $\mathrm{C}(32)-\mathrm{Ru}(1)-\mathrm{C}(1)-\mathrm{C}(2)$ & $-155.34(12)$ \\
\hline $\mathrm{C}(4)-\mathrm{Ru}(1)-\mathrm{C}(1)-\mathrm{C}(2)$ & $-83.43(12)$ \\
\hline $\mathrm{C}(3)-\mathrm{Ru}(1)-\mathrm{C}(1)-\mathrm{C}(2)$ & $-39.86(11)$ \\
\hline $\mathrm{N}(1)-\mathrm{Ru}(1)-\mathrm{C}(1)-\mathrm{C}(2)$ & $122.32(12)$ \\
\hline $\mathrm{C}(5)-\mathrm{Ru}(1)-\mathrm{C}(1)-\mathrm{C}(2)$ & $-122.80(16)$ \\
\hline $\mathrm{O}(1)-\mathrm{C}(1)-\mathrm{C}(2)-\mathrm{C}(3)$ & $179.51(19)$ \\
\hline$C(5)-C(1)-C(2)-C(3)$ & $11.1(2)$ \\
\hline $\mathrm{Ru}(1)-\mathrm{C}(1)-\mathrm{C}(2)-\mathrm{C}(3)$ & $63.88(13)$ \\
\hline $\mathrm{O}(1)-\mathrm{C}(1)-\mathrm{C}(2)-\mathrm{C}(6)$ & $-8.0(3)$ \\
\hline $\mathrm{C}(5)-\mathrm{C}(1)-\mathrm{C}(2)-\mathrm{C}(6)$ & $-176.42(18)$ \\
\hline $\mathrm{Ru}(1)-\mathrm{C}(1)-\mathrm{C}(2)-\mathrm{C}(6)$ & $-123.67(19)$ \\
\hline $\mathrm{O}(1)-\mathrm{C}(1)-\mathrm{C}(2)-\mathrm{Ru}(1)$ & $115.6(2)$ \\
\hline $\mathrm{C}(5)-\mathrm{C}(1)-\mathrm{C}(2)-\mathrm{Ru}(1)$ & $-52.75(13)$ \\
\hline $\mathrm{C}(33)-\mathrm{Ru}(1)-\mathrm{C}(2)-\mathrm{C}(3)$ & $86.44(12)$ \\
\hline $\mathrm{C}(32)-\mathrm{Ru}(1)-\mathrm{C}(2)-\mathrm{C}(3)$ & $-35.5(3)$ \\
\hline $\mathrm{C}(4)-\mathrm{Ru}(1)-\mathrm{C}(2)-\mathrm{C}(3)$ & $-37.50(11)$ \\
\hline $\mathrm{N}(1)-\mathrm{Ru}(1)-\mathrm{C}(2)-\mathrm{C}(3)$ & $-170.42(11)$ \\
\hline $\mathrm{C}(5)-\mathrm{Ru}(1)-\mathrm{C}(2)-\mathrm{C}(3)$ & $-80.05(12)$ \\
\hline$C(1)-R u(1)-C(2)-C(3)$ & $-114.08(16)$ \\
\hline $\mathrm{C}(33)-\mathrm{Ru}(1)-\mathrm{C}(2)-\mathrm{C}(6)$ & $-35.22(16)$ \\
\hline $\mathrm{C}(32)-\mathrm{Ru}(1)-\mathrm{C}(2)-\mathrm{C}(6)$ & $-157.2(2)$ \\
\hline$C(4)-R u(1)-C(2)-C(6)$ & $-159.16(18)$ \\
\hline $\mathrm{C}(3)-\mathrm{Ru}(1)-\mathrm{C}(2)-\mathrm{C}(6)$ & $-121.7(2)$ \\
\hline $\mathrm{N}(1)-\mathrm{Ru}(1)-\mathrm{C}(2)-\mathrm{C}(6)$ & $67.92(16)$ \\
\hline $\mathrm{C}(5)-\mathrm{Ru}(1)-\mathrm{C}(2)-\mathrm{C}(6)$ & $158.29(17)$ \\
\hline
\end{tabular}

$\mathrm{C}(1)-\mathrm{Ru}(1)-\mathrm{C}(2)-\mathrm{C}(6)$

$\mathrm{C}(33)-\mathrm{Ru}(1)-\mathrm{C}(2)-\mathrm{C}(1)$

$\mathrm{C}(32)-\mathrm{Ru}(1)-\mathrm{C}(2)-\mathrm{C}(1)$

$\mathrm{C}(4)-\mathrm{Ru}(1)-\mathrm{C}(2)-\mathrm{C}(1)$

$\mathrm{C}(3)-\mathrm{Ru}(1)-\mathrm{C}(2)-\mathrm{C}(1)$

$\mathrm{N}(1)-\mathrm{Ru}(1)-\mathrm{C}(2)-\mathrm{C}(1)$

$\mathrm{C}(5)-\mathrm{Ru}(1)-\mathrm{C}(2)-\mathrm{C}(1)$

$\mathrm{C}(6)-\mathrm{C}(2)-\mathrm{C}(3)-\mathrm{C}(4)$

$\mathrm{C}(1)-\mathrm{C}(2)-\mathrm{C}(3)-\mathrm{C}(4)$

$\mathrm{Ru}(1)-\mathrm{C}(2)-\mathrm{C}(3)-\mathrm{C}(4)$

$\mathrm{C}(6)-\mathrm{C}(2)-\mathrm{C}(3)-\mathrm{C}(12)$

$\mathrm{C}(1)-\mathrm{C}(2)-\mathrm{C}(3)-\mathrm{C}(12)$

$\mathrm{Ru}(1)-\mathrm{C}(2)-\mathrm{C}(3)-\mathrm{C}(12)$

$\mathrm{C}(6)-\mathrm{C}(2)-\mathrm{C}(3)-\mathrm{Ru}(1)$

$\mathrm{C}(1)-\mathrm{C}(2)-\mathrm{C}(3)-\mathrm{Ru}(1)$

$\mathrm{C}(33)-\mathrm{Ru}(1)-\mathrm{C}(3)-\mathrm{C}(4)$

$\mathrm{C}(32)-\mathrm{Ru}(1)-\mathrm{C}(3)-\mathrm{C}(4)$

$\mathrm{N}(1)-\mathrm{Ru}(1)-\mathrm{C}(3)-\mathrm{C}(4)$

$\mathrm{C}(2)-\mathrm{Ru}(1)-\mathrm{C}(3)-\mathrm{C}(4)$

$\mathrm{C}(5)-\mathrm{Ru}(1)-\mathrm{C}(3)-\mathrm{C}(4)$

$\mathrm{C}(1)-\mathrm{Ru}(1)-\mathrm{C}(3)-\mathrm{C}(4)$

$\mathrm{C}(33)-\mathrm{Ru}(1)-\mathrm{C}(3)-\mathrm{C}(2)$

$\mathrm{C}(32)-\mathrm{Ru}(1)-\mathrm{C}(3)-\mathrm{C}(2)$

$\mathrm{C}(4)-\mathrm{Ru}(1)-\mathrm{C}(3)-\mathrm{C}(2)$

$\mathrm{N}(1)-\mathrm{Ru}(1)-\mathrm{C}(3)-\mathrm{C}(2)$

$\mathrm{C}(5)-\mathrm{Ru}(1)-\mathrm{C}(3)-\mathrm{C}(2)$

$\mathrm{C}(1)-\mathrm{Ru}(1)-\mathrm{C}(3)-\mathrm{C}(2)$

$\mathrm{C}(33)-\mathrm{Ru}(1)-\mathrm{C}(3)-\mathrm{C}(12)$

$\mathrm{C}(32)-\mathrm{Ru}(1)-\mathrm{C}(3)-\mathrm{C}(12)$

$\mathrm{C}(4)-\mathrm{Ru}(1)-\mathrm{C}(3)-\mathrm{C}(12)$

$\mathrm{N}(1)-\mathrm{Ru}(1)-\mathrm{C}(3)-\mathrm{C}(12)$

$\mathrm{C}(2)-\mathrm{Ru}(1)-\mathrm{C}(3)-\mathrm{C}(12)$

$\mathrm{C}(5)-\mathrm{Ru}(1)-\mathrm{C}(3)-\mathrm{C}(12)$

$\mathrm{C}(1)-\mathrm{Ru}(1)-\mathrm{C}(3)-\mathrm{C}(12)$

$\mathrm{C}(2)-\mathrm{C}(3)-\mathrm{C}(4)-\mathrm{C}(5)$

$\mathrm{C}(12)-\mathrm{C}(3)-\mathrm{C}(4)-\mathrm{C}(5)$

$\mathrm{Ru}(1)-\mathrm{C}(3)-\mathrm{C}(4)-\mathrm{C}(5)$

$\mathrm{C}(2)-\mathrm{C}(3)-\mathrm{C}(4)-\mathrm{C}(19)$

$\mathrm{C}(12)-\mathrm{C}(3)-\mathrm{C}(4)-\mathrm{C}(19)$

$\mathrm{Ru}(1)-\mathrm{C}(3)-\mathrm{C}(4)-\mathrm{C}(19)$

$\mathrm{C}(2)-\mathrm{C}(3)-\mathrm{C}(4)-\mathrm{Ru}(1)$

$\mathrm{C}(12)-\mathrm{C}(3)-\mathrm{C}(4)-\mathrm{Ru}(1)$

$\mathrm{C}(33)-\mathrm{Ru}(1)-\mathrm{C}(4)-\mathrm{C}(3)$

$\mathrm{C}(32)-\mathrm{Ru}(1)-\mathrm{C}(4)-\mathrm{C}(3)$

$\mathrm{N}(1)-\mathrm{Ru}(1)-\mathrm{C}(4)-\mathrm{C}(3)$

$\mathrm{C}(2)-\mathrm{Ru}(1)-\mathrm{C}(4)-\mathrm{C}(3)$

$\mathrm{C}(5)-\mathrm{Ru}(1)-\mathrm{C}(4)-\mathrm{C}(3)$

$\mathrm{C}(1)-\mathrm{Ru}(1)-\mathrm{C}(4)-\mathrm{C}(3)$

$\mathrm{C}(33)-\mathrm{Ru}(1)-\mathrm{C}(4)-\mathrm{C}(5)$

$\mathrm{C}(32)-\mathrm{Ru}(1)-\mathrm{C}(4)-\mathrm{C}(5)$

$\mathrm{C}(3)-\mathrm{Ru}(1)-\mathrm{C}(4)-\mathrm{C}(5)$

$\mathrm{N}(1)-\mathrm{Ru}(1)-\mathrm{C}(4)-\mathrm{C}(5)$

$\mathrm{C}(2)-\mathrm{Ru}(1)-\mathrm{C}(4)-\mathrm{C}(5)$
124.3(2)

$-159.47(11)$

$78.5(3)$

$76.59(12)$

$114.08(16)$

$-56.33(11)$

34.04(11)

$179.80(18)$

$-8.0(2)$

61.60(13)

$-11.3(3)$

$160.92(18)$

$-129.53(19)$

118.2(2)

$-69.56(13)$

$144.22(12)$

50.11(14)

$-104.15(12)$

$-117.63(16)$

$-37.83(11)$

$-79.16(12)$

$-98.15(12)$

167.75(12)

117.63(16)

13.49(16)

$79.80(12)$

$38.47(11)$

24.6(2)

$-69.5(2)$

$-119.6(2)$

136.27(17)

122.8(2)

$-157.4(2)$

161.3(2)

1.5(2)

$-167.50(18)$

64.43(13)

169.12(18)

$0.1(3)$

$-128.0(2)$

$-62.91(13)$

128.07(19)

$-44.76(14)$

$-141.94(11)$

113.51(11)

37.49(11)

116.86(16)

78.98(12)

$-161.62(12)$

$101.20(12)$

$-116.86(16)$

$-3.35(16)$

$-79.37(12)$ 


$\begin{array}{lc}\mathrm{C}(3)-\mathrm{Ru}(1)-\mathrm{C}(33)-\mathrm{O}(2) & -6.5(17) \\ \mathrm{N}(1)-\mathrm{Ru}(1)-\mathrm{C}(33)-\mathrm{O}(2) & -144.4(17) \\ \mathrm{C}(2)-\mathrm{Ru}(1)-\mathrm{C}(33)-\mathrm{O}(2) & -44.5(17) \\ \mathrm{C}(5)-\mathrm{Ru}(1)-\mathrm{C}(33)-\mathrm{O}(2) & -11.3(18) \\ \mathrm{C}(1)-\mathrm{Ru}(1)-\mathrm{C}(33)-\mathrm{O}(2) & -60.9(17) \\ \mathrm{C}(41)-\mathrm{N}(1)-\mathrm{C}(34)-\mathrm{C}(35) & 60.0(2) \\ \mathrm{Ru}(1)-\mathrm{N}(1)-\mathrm{C}(34)-\mathrm{C}(35) & -159.13(13) \\ \mathrm{N}(1)-\mathrm{C}(34)-\mathrm{C}(35)-\mathrm{C}(40) & -94.9(2) \\ \mathrm{N}(1)-\mathrm{C}(34)-\mathrm{C}(35)-\mathrm{C}(36) & 86.6(2) \\ \mathrm{C}(40)-\mathrm{C}(35)-\mathrm{C}(36)-\mathrm{C}(37) & -0.3(3) \\ \mathrm{C}(34)-\mathrm{C}(35)-\mathrm{C}(36)-\mathrm{C}(37) & 178.28(19) \\ \mathrm{C}(35)-\mathrm{C}(36)-\mathrm{C}(37)-\mathrm{C}(38) & 0.7(3) \\ \mathrm{C}(36)-\mathrm{C}(37)-\mathrm{C}(38)-\mathrm{C}(39) & 0.1(3)\end{array}$

$\begin{array}{lc}\mathrm{C}(37)-\mathrm{C}(38)-\mathrm{C}(39)-\mathrm{C}(40) & -1.2(3) \\ \mathrm{C}(36)-\mathrm{C}(35)-\mathrm{C}(40)-\mathrm{C}(39) & -0.8(3) \\ \mathrm{C}(34)-\mathrm{C}(35)-\mathrm{C}(40)-\mathrm{C}(39) & -179.35(19) \\ \mathrm{C}(38)-\mathrm{C}(39)-\mathrm{C}(40)-\mathrm{C}(35) & 1.6(3) \\ \mathrm{C}(34)-\mathrm{N}(1)-\mathrm{C}(41)-\mathrm{C}(42) & -166.01(15) \\ \mathrm{Ru}(1)-\mathrm{N}(1)-\mathrm{C}(41)-\mathrm{C}(42) & 58.86(18) \\ \mathrm{C}(34)-\mathrm{N}(1)-\mathrm{C}(41)-\mathrm{C}(43) & 69.10(17) \\ \mathrm{Ru}(1)-\mathrm{N}(1)-\mathrm{C}(41)-\mathrm{C}(43) & -66.03(17) \\ \mathrm{N}(1)-\mathrm{C}(41)-\mathrm{C}(42)-\mathrm{C}(43) \# 1 & 176.28(12) \\ \mathrm{C}(43)-\mathrm{C}(41)-\mathrm{C}(42)-\mathrm{C}(43) \# 1 & -57.51(16) \\ \mathrm{N}(1)-\mathrm{C}(41)-\mathrm{C}(43)-\mathrm{C}(42) \# 1 & -178.03(13) \\ \mathrm{C}(42)-\mathrm{C}(41)-\mathrm{C}(43)-\mathrm{C}(42) \# 1 & 57.18(17)\end{array}$

Symmetry transformations used to generate equivalent atoms: $\# 1-\mathrm{x},-\mathrm{y}+2,-\mathrm{z}+2$

Table 7. Hydrogen bonds for $\mathbf{2 3} \bullet\left(\mathbf{C H}_{\mathbf{2}} \mathbf{C l}_{\mathbf{2}}\right)_{2}\left[\AA\right.$ and $\left.{ }^{\circ}\right]$.

\begin{tabular}{lcccc}
\hline $\mathrm{D}-\mathrm{H} \ldots \mathrm{A}$ & $\mathrm{d}(\mathrm{D}-\mathrm{H})$ & $\mathrm{d}(\mathrm{H} \ldots \mathrm{A})$ & $\mathrm{d}(\mathrm{D} \ldots \mathrm{A})$ & $<$ (DHA) \\
\hline $\mathrm{N}(1)-\mathrm{H}(1) \ldots \mathrm{O}(1)$ & 0.93 & 2.07 & $2.884(2)$ & 145.2
\end{tabular}

Symmetry transformations used to generate equivalent atoms: \#1 -x,-y+2,-z+2 
Selected ${ }^{15} \mathrm{~N}$ NMR Spectra

${ }^{15} \mathrm{~N}$ NMR at $0{ }^{\circ} \mathrm{C}$ in toluene- $d_{8}$

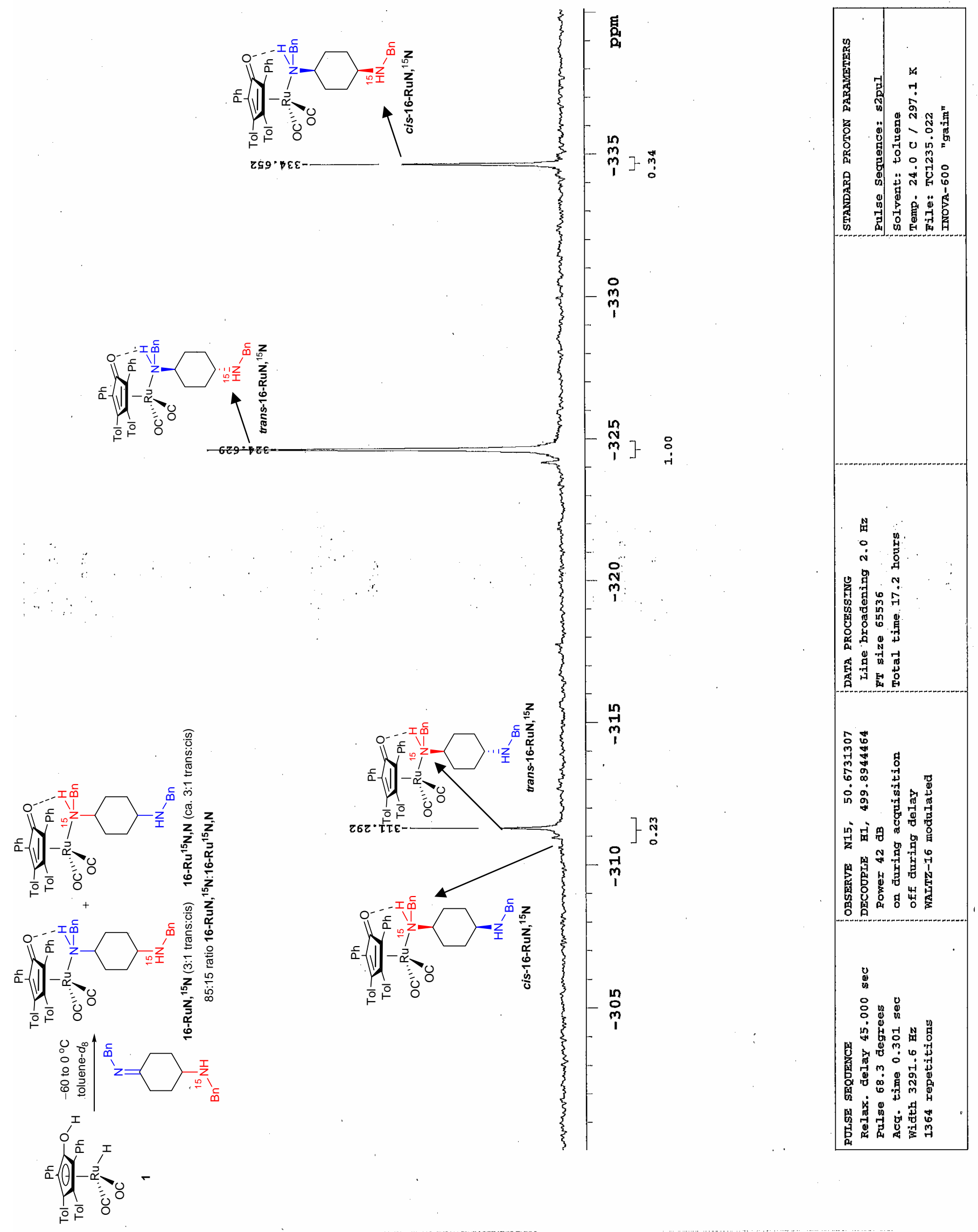


${ }^{15} \mathrm{~N}$ NMR at $0{ }^{\circ} \mathrm{C}$ in toluene- $d_{8}$ after $4 \mathrm{~h}$ at $50{ }^{\circ} \mathrm{C}$
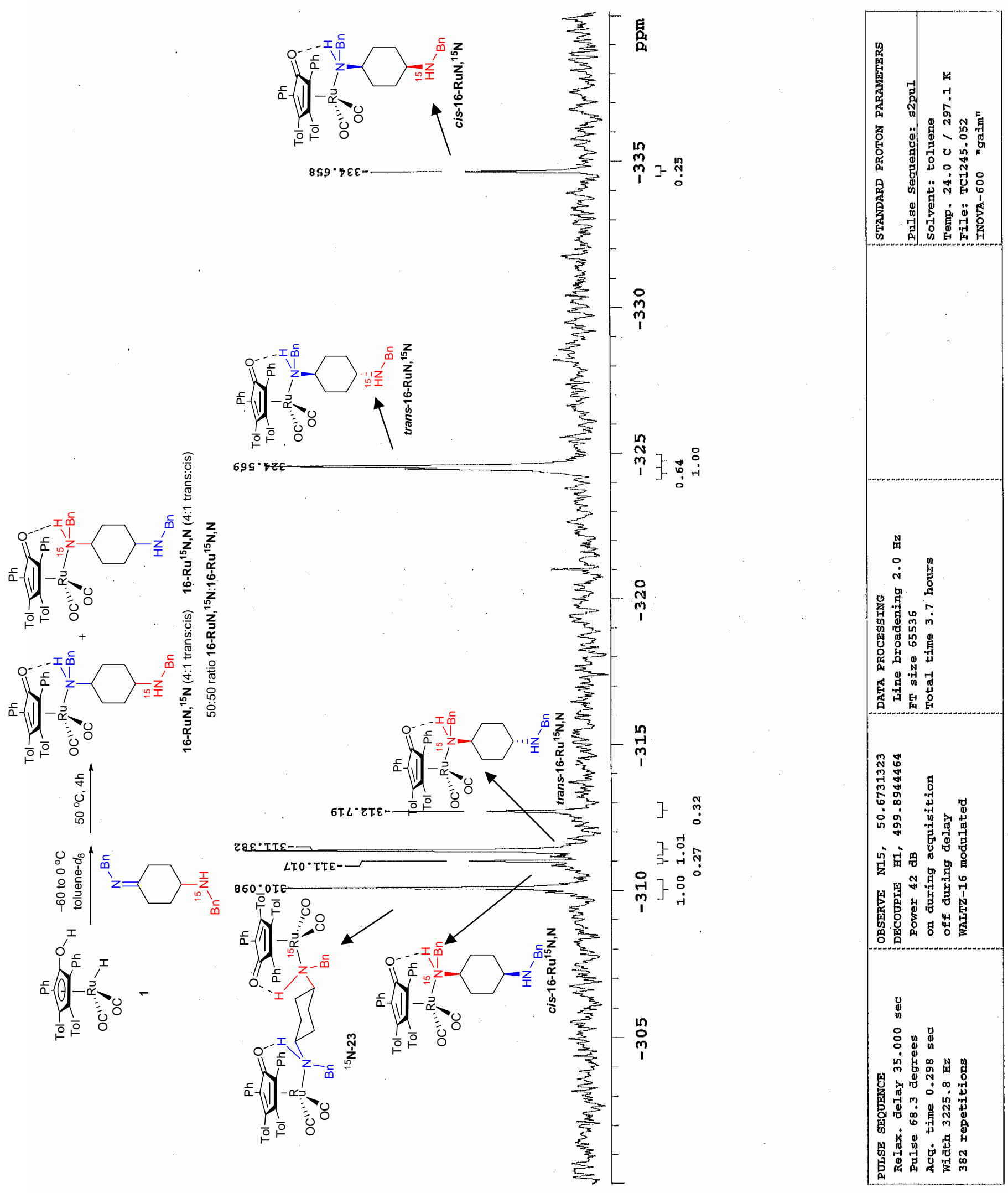
$2 \mathrm{D}{ }^{15} \mathrm{~N}-1 \mathrm{H}$ HSQC NMR of cis-16 in toluene- $d_{8}$

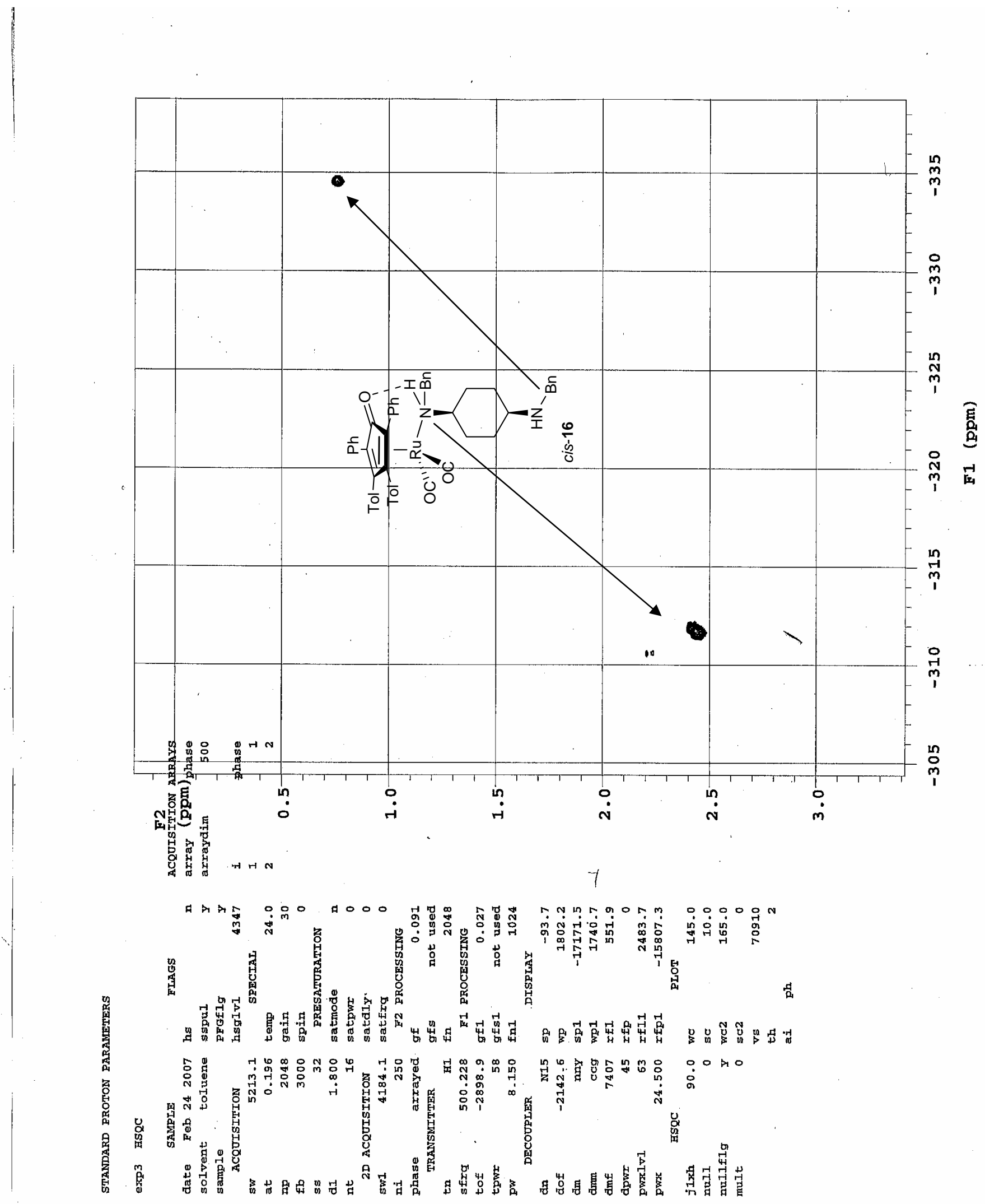

